\title{
Quantum Dynamical Behaviour in Complex Systems - A Semiclassical
} Approach

\author{
by \\ Nandini Ananth \\ M.Sc (Indian Institute of Technology Madras, Chennai) 2003 \\ A dissertation submitted in partial satisfaction of the \\ requirements for the degree of \\ Doctor of Philosophy \\ in \\ Chemistry \\ in the \\ GRADUATE DIVISION \\ of the \\ UNIVERSITY OF CALIFORNIA, BERKELEY
}

Committee in charge:

Professor William H. Miller, Chair

Professor Robert A. Harris

Professor Robert G. Littlejohn

Spring 2008 


\section{Quantum Dynamical Behaviour in Complex Systems - A Semiclassical}

\section{Approach}

Copyright 2008

by

Nandini Ananth

This work was supported by the Office of Naval Research Grant No.

N00014-07-1-0586 and by the Director, Office of Science, Office of Basic Energy

Sciences, Chemical Sciences, Geosciences, and Biosciences Division, U.S.

Department of Energy under Contract No. DE-AC02-05CH11231. 


\begin{abstract}
by

Nandini Ananth

Doctor of Philosophy in Chemistry

University of California, Berkeley

Professor William H. Miller, Chair
\end{abstract}

Quantum Dynamical Behaviour in Complex Systems - A Semiclassical Approach

One of the biggest challenges in Chemical Dynamics is describing the behavior of complex systems accurately. Classical MD simulations have evolved to a point where calculations involving thousands of atoms are routinely carried out. Capturing coherence, tunneling and other such quantum effects for these systems, however, has proven considerably harder.

Semiclassical methods such as the Initial Value Representation (SC-IVR) provide a practical way to include quantum effects while still utilizing only classical trajectory information. For smaller systems, this method has been proven to be most effective, encouraging the hope that it can be extended to deal with a large number of degrees of freedom. Several variations upon the original idea of the SCIVR have been developed to help make these larger calculations more tractable; 
these range from the simplest, classical limit form, the Linearized IVR (LSC-IVR) to the quantum limit form, the Exact Forward-Backward version (EFB-IVR).

In this thesis a method to tune between these limits is described which allows us to choose exactly which degrees of freedom we wish to treat in a more quantum mechanical fashion and to what extent. This formulation is called the Tuning IVR (TIVR).

We further describe methodology being developed to evaluate the prefactor term that appears in the IVR formalism. The regular prefactor is composed of the Monodromy matrices (jacobians of the transformation from initial to finial coordinates and momenta) which are time evolved using the Hessian. Standard MD simulations require the potential surfaces and their gradients, but very rarely is there any information on the second derivative.

We would like to be able to carry out the SC-IVR calculation without this information too. With this in mind a finite difference scheme to obtain the Hessian on-the-fly is proposed.

We also apply the IVR formalism to a few problems of current interest. A method to obtain energy eigenvalues accurately for complex systems is described. We proposed the use of a semiclassical correction term to a preliminary quantum calculation using, for instance, a variational approach. This allows us to increase the accuracy significantly.

Modeling Nonadiabatic dynamics has always been a challenge to classical sim- 
ulations because the multi-state nature of the dynamics cannot be described accurately by the time evolution on a single average surface, as is the classical approach. We show that using the Meyer-Miller-Stock-Thoss(MMST) representation of the exact vibronic Hamiltonian in combination with the IVR allows us to accurately describe dynamics where the non Born-Oppenheimer regime.

One final problem that we address is that of extending this method to the long time regime. We propose the use of a time independent sampling function in the Monte Carlo integration over the phase space of initial trajectory conditions. This allows us to better choose the regions of importance at the various points in time; by using more trajectories in the important regions, we show that the integration can be converged much easier. An algorithm based loosely on the methods of Diffusion Monte Carlo is developed that allows us to carry out this time dependent sampling in a most efficient manner.

Professor William H. Miller

Dissertation Committee Chair 
To Amma, Appa and Sudarsh 


\section{Contents}

List of Figures $\quad$ v

List of Tables viii

1 Introduction 1

I The Semiclassical Idea . . . . . . . . . . . . . . . . . . . 1

I.1 The WKB Theory . . . . . . . . . . . . . . 3

I.2 The Van Vleck Propagator . . . . . . . . . . . . . . 5

I.3 Gaussian Wavepackets . . . . . . . . . . . . . . 6

II Thesis Outline . . . . . . . . . . . . . . . . . . . 8

2 The Semiclassical Initial Value Representation(SC-IVR) 11

I Introduction . . . . . . . . . . . . . . . . . . . . 11

II The IVR Trick . . . . . . . . . . . . . . . . . . . . 13

II.1 Herman Kluk IVR (HK-IVR) . . . . . . . . . . . . 15

III Correlation Functions . . . . . . . . . . . . . . . . . . 17

III.1 Double HK-IVR (DHK-IVR) . . . . . . . . . . . . 18

III.2 Exact Forward-Backward IVR (EFB-IVR) . . . . . . . . . 20

III.3 The Forward Backward (FB-IVR) . . . . . . . . . . . . 22

III.4 LSC-IVR . . . . . . . . . . . . . . . . . . . . . . . . 25

3 A Semiclassical Correction Term for Energy Eigenvalues 27

I Introduction . . . . . . . . . . . . . . . . . . . . 27

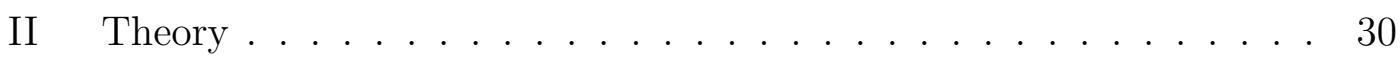

II.1 SC correction to the S-Matrix . . . . . . . . . . . . 30

II.2 SC Correction for the Energy . . . . . . . . . . . . . . . 34

III Application ........................ . . . 41

III.1 The Anharmonic Oscillator Model . . . . . . . . . . . . . . . 43

III.2 The Quartic Oscillator . . . . . . . . . . . . . . . . . 45

III.3 The Harmonium Atom - A Two Electron Model . . . . . . . 46 
IV Discussion . . . . . . . . . . . . . . . . . . . . . 49

IV.1 Anharmonic Oscillator . . . . . . . . . . . . . . . 49

IV.2 Quartic Oscillator . . . . . . . . . . . . . . . 55

IV.3 Harmonium Atom . . . . . . . . . . . . . . . . . . 56

4 Accessing Metastable States and Long Time Dynamics - Time $\begin{array}{ll}\text { Dependent Monte Carlo Sampling } & 64\end{array}$

I Introduction . . . . . . . . . . . . . . . . . . . . . . . . . . 64

II Theory ....................... 65

II.1 The Normalization Constant . . . . . . . . . . . . . . . . 68

II.2 The Sampling Function . . . . . . . . . . . . . . . . . 70

II.3 The Sampling Algorithm . . . . . . . . . . . . . . . . . . . 71

III Applications . . . . . . . . . . . . . . . . . . . 73

III.1 1D Ozone . . . . . . . . . . . . . . . . . . . 73

III.2 Water . . . . . . . . . . . . . . . . . . . 74

IV Discussion .............................. 74

5 Semiclassical Approach to Nonadiabatic Dynamics $\quad 80$

I Introduction . . . . . . . . . . . . . . . . . 80

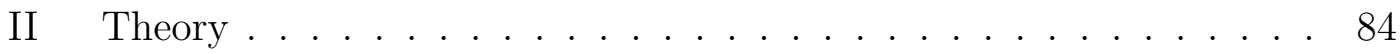

II.1 The Ehrenfest Method . . . . . . . . . . . . . . . . . . . 84

II.2 The Meyer-Miller-Stock-Thoss (MMST) Hamiltonian . . . . 86

III Applications . . . . . . . . . . . . . . . . . . . . . 90

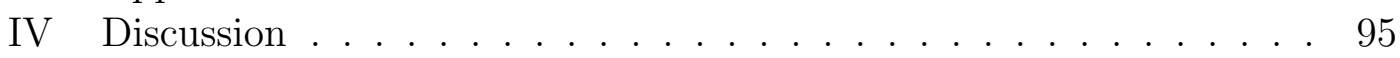

V Future Work . . . . . . . . . . . . . . . . . . 103

6 Tuning between the Quantum and Classical Limits of SC-IVR -

$\begin{array}{lr}\text { Filinov Transformation } & 104\end{array}$

I Introduction . . . . . . . . . . . . . . . . . . . . . . . 104

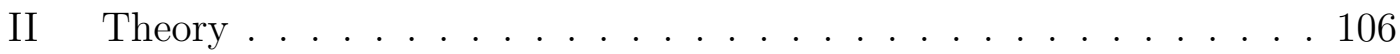

II.1 Modified Filinov Transformation . . . . . . . . . . . . . . 106

II.2 GFB-IVR . . . . . . . . . . . . . . . . . . . . . . . 108

II.3 A Tuning IVR (TIVR) . . . . . . . . . . . . . . . . . . . . . 109

III Applications . . . . . . . . . . . . . . . . . . . . 112

III.1 Anharmonic Oscillator . . . . . . . . . . . . . . . . . . . . 112

III.2 System-Bath Model . . . . . . . . . . . . . . . . . . . . . . . 113

III.3 Tracking the Prefactor . . . . . . . . . . . . . . . . . . . . . 114

IV Discussion . . . . . . . . . . . . . . . . . . . . 115

V Future Work . . . . . . . . . . . . . . . . . . 118 
7 The Semiclassical Prefactor - Exploring Alternate Evaluation Methods 120

I Introduction . . . . . . . . . . . . . . . . . . . 120

II Theory . . . . . . . . . . . . . . . . . . . . . 122

II.1 Semiclassical Prefactor . . . . . . . . . . . . . . . . . 122

II.2 Log-Derivative Method . . . . . . . . . . . . . . . . . . . 123

II.3 Finite Difference - Light Method . . . . . . . . . . . . . 125

II.4 Finite Difference in Time . . . . . . . . . . . . . . . . . . . 127

III Discussion . . . . . . . . . . . . . . . . . . . . . . 130

IV Future Work . . . . . . . . . . . . . . . . . 136

$\begin{array}{lll}8 & \text { Conclusion } & 137\end{array}$

$\begin{array}{ll}\text { Bibliography } & 140\end{array}$ 


\section{List of Figures}

2.1 Non-linear boundary value problem . . . . . . . . . . . . . . 14

2.2 Showing the different branches of the function $q_{2}\left(p_{1}\right) \ldots \ldots \ldots 14$

2.3 Schematic representation of the path described by the classical trajectory in our simulation. . . . . . . . . . . . . . . . . 19

3.1 The intersection of $\mathrm{F}(\mathrm{E})$ and $\mathrm{E}$ for the $\epsilon=0.01$ case . . . . . . 49

3.2 The intersection of $\mathrm{F}(\mathrm{E})$ and $\mathrm{E}$ for the $\epsilon=0.01$ case - Figure 3.1 over a larger energy window . . . . . . . . . . . . . 51

3.3 The same as Figure 3.1 but with a bad guess for the 'variational' wavefunction with $\gamma=1.5$ and $\epsilon=0.01 \ldots \ldots \ldots 2$

3.4 Anharmonic oscillator $\gamma=2$ and over a range of $\epsilon$ values. We look to see how much the accuracy of the calculation is affected by changing these values. . . . . . . . . . . . . . . . . . . . 53

3.5 Zoomed in version of Figure $3.4 \ldots \ldots \ldots \ldots$. . . . . . . 54

$3.6 \quad$ Energy of a Quartic Oscillator with $\gamma=1.61$ and $\epsilon=0.001 . \quad \ldots \quad 55$

3.7 Quartic oscillator with $\gamma=1.61$ and $\epsilon=0.001$ with several $\epsilon$ values. $\quad 57$

3.8 Figure 3.7 Zoomed in to see intersection point. . . . . . . . . . 58

3.9 Energy intersection for Harmonium Atom with $\mathrm{k}=0.25$ and $\epsilon=0.00159$

3.10 Harmonium atom of Figure 3.9 zoomed in. . . . . . . . . . . 60

3.11 Energy intersection for Harmonium Atom with $\mathrm{k}=0.32$ and $\epsilon=0.00160$

3.12 Harmonium atom of Figure 3.11 zoomed in. . . . . . . . . . 61

3.13 Energy intersection for Harmonium Atom with $\mathrm{k}=5.1$ and $\epsilon=0.01 .62$

3.14 Harmonium Atom of Figure 3.13 zoomed in. . . . . . . . . . 63

4.1 Real part of autocorrelation function for Ozone. The dashed line represents the regular SC-IVR calculation and the time dependent sampling method is the full line . . . . . . . . . . . . . . 77

4.2 Imaginary part of autocorrelation function for Ozone. The dashed line represents the regular SC-IVR calculation and the time dependent sampling method is the full line . . . . . . . . . . . . . 77 
4.3 The Normalization constant is obtained exactly (full line) or with the Log-Derivative method. . . . . . . . . . . . . . . . . 78

4.4 The Normalization constant is obtained exactly (full line) or with the Log-Derivative method (dashed line). . . . . . . . . . . . 78

4.5 Imaginary part of the autocorrelation function for the 3D model of water. The dashed line is the regular sampling method and the full line is time dependent sampling. . . . . . . . . . . . . .

4.6 Real part of autocorrelation function for the 3D model of water. The dashed line is the regular sampling method and the full line is the time dependent sampling. . . . . . . . . . . . .

5.1 The Potential Curves in both Diabatic and Adiabatic Representation for Model 1. . . . . . . . . . . . . . . . . . . 92

5.2 The Potential Curves in both Diabatic and Adiabatic Representation for Model 2 . . . . . . . . . . . . . . . . . . . . 93

5.3 Comparison of results of FB-IVR, Exact FB-IVR and exact Quantum calculations for Model 1 with an initial energy of 0.03 Hartree . 96

5.4 Comparison of results of FB-IVR, Exact FB-IVR and exact Quantum calculations for Model 1 with an initial energy of 0.1 Hartree . 97

5.5 Comparison of results of FB-IVR, Exact FB-IVR and exact Quantum calculations for Model 2 with an initial energy of 0.03 Hartree . 98

5.6 Comparison of results of FB-IVR, Exact FB-IVR and exact Quantum calculations for Model 2 with an initial energy of 0.1 Hartree . 98

5.7 Linearised IVR and Ehrenfest results for Model 1. Initial Energy = 0.03 Hartree . . . . . . . . . . . . . . . . . . . . . . . . 100

5.8 Linearised IVR and Ehrenfest results for Model 1. Initial Energy = 0.1 Hartree . . . . . . . . . . . . . . . . . . . . . 100

5.9 Linearised IVR and Ehrenfest results for Model 2. Initial Energy = 0.03 Hartree . . . . . . . . . . . . . . . . . . . . . . . 101

5.10 Linearised IVR and Ehrenfest results for Model 2. Initial Energy = 0.1 Hartree . . . . . . . . . . . . . . . . . . . . 101

5.11 Comparison of the Adiabatic and Diabatic results for Model 1, with an initial energy 0.03 Hartree . . . . . . . . . . . . . . . . . 102

5.12 Comparison of the Adiabatic and Diabatic results for Model 1, with an initial energy 0.1 Hartree . . . . . . . . . . . . . . . . . . . 102

6.1 Comparison of the position correlation function for an Anharmonic oscillator obtained using DHK-IVR, EFB-IVR and the exact DVR . 116

6.2 Examining the $\gamma_{t} \rightarrow \infty$ limit of the DHK-IVR with the EFB-IVR calculation. . . . . . . . . . . . . . . . . 117

6.3 The Quantum limit of TIVR - comparing the $\mathbf{c}_{\mathbf{p}} \rightarrow 0$ limit with the EFB-IVR . . . . . . . . . . . . . . . 117 
6.4 The classical limit of the TIVR - contrasting the LSC-IVR result with the limit $\mathbf{c}_{\mathbf{p}} \rightarrow \infty \ldots \ldots \ldots \ldots$

6.5 The position correlation for an anharmonic system coupled to 10 harmonic bath modes; The limits of a large and a small value of $c p$ are compared. . . . . . . . . . . . . . . . . . . . . 119

7.1 The prefactor for a simple 1D Morse potential; the red line is the HK prefactor with an analytic Hessian; the blue and green dotted lines are the log-derivative and HK prefactors respectively with a finite difference Hessian. . . . . . . . . . . . . . . . . . . . . . . . 131

7.2 Comparing the diagonal elements of the analytic(red) Hessian and the finite difference (green) Hessian. . . . . . . . . . . . . . . . . 132

7.3 Comparing the off-diagonal elements of the analytic(red) Hessian and the finite difference (green) Hessian. . . . . . . . . . . . 133

7.4 The momentum, the second derivative of the momentum in time and the force constant along the course of the trajectory . . . . . . 134

7.5 The autocorrelation function using the regular HK-IVR prefactor, and the WKB prefactor calculated with the analytic Hessian(black) and the finite difference Hessian (red) . . . . . . . . . . . 135 


\section{List of Tables}

3.1 Results for the anharmonic oscillator with $\gamma=2$ and $\epsilon=0.01$. . . 50

3.2 Results for anharmonic oscillator with $\gamma=1.5$ and $\epsilon=0.01 \ldots$. . . 57

3.3 Results for the Quartic Oscillator with $\gamma=1.61$ and $\epsilon=0.001$. . . 57

3.4 Results for the Harmonium Atom with different $k$ values . . . . . . 58 


\section{Acknowledgments}

I have had a wonderful time here at Berkeley and would like to acknowledge here all the people who have made such a difference to my life both in and out of the academic setting.

I have had the great privilege of working with Prof. Bill Miller on the research described in this thesis. I have learnt at lot from him through one-on-one discussions as well as at group meeting on everything from Quantum Mechanics to the origins of the song 'American Pie'! I have also thoroughly enjoyed sitting through the classes he has taught - the little insightful observations that are so casually scattered throughout bring clarity and lend perspective to the subject under consideration. I should also mention here the boundless patience he has had in answering my many questions on any topic and at all times of the day.

As someone with aspirations to an academic career, I greatly admire Prof.Miller's dedication to his work; his appreciation of mathematical rigor and elegance is coupled with a breadth of knowledge that allows for a better understanding of the underlying physical process, a quality I hope to be able to emulate in my own work. I feel lucky to have had the chance to apprentice, as it were, with someone so skilled at the art of science.

I also have had the pleasure of taking a Quantum class with Prof. Bob Harris and his unique perspective on the subject made for a most enjoyable experience. Since then we have had several interesting conversations about science and it is 
always inspiring and exciting to hear what Prof.Harris is thinking about or working on currently!

I would like to further thank my advisor for my Masters thesis - Prof. N. Chandrakumar. His contagious enthusiasm for Chemistry and Quantum Mechanics played a big role in my decision to pursue academics in this area. I would also like to thank Prof. K. L. Sebastian with whom I had the chance to work on a summer project. I had a very enjoyable experience in his lab which was my first exposure to a research environment as well as Semiclassical Mechanics.

I would also like to thank members of the Miller group - both past and present for their support at work as well as their friendship. Yimin helped me get started with work in this area, spending a lot of his valuable time teaching a rookie programmer what to do. I was also lucky to work with him on a project and learnt a lot from that experience. I have also worked with Charu and enjoyed our many discussions on research as well as life in Madras. Mike, my batch-mate, has been a good friend from my first day here and we have had some fun times together stumbling our way through the maze of graduate school do's and dont's all the way to the end! Shervin and David who have both been my office mates at various times and been great for discussing research with and have become good friends both in and out of the office. Cristian, Jian, Tommy and Guohua were/are postdocs with whom I have had many interesting and useful discussions about research which have been most enlightening. I would also like to thank Jiri, Richard, Michele, 
Sandy and Atsushi. I am also very grateful to Cheryn who took care of all the administrative details that came up and is an incredible source of information about the school and Berkeley itself and also a friend with whom it is always a pleasure to stop and chat.

I am very fortunate to have the best family anyone can ask for. My parents and my brother are my role models, each in their own way and everything I have been able to do I owe to their constant love and support.

Finally, I would like to thank my friends here at Berkeley who have shared so many experiences with me - Brad, Jiyeon, Karma, Jacob, James, Idalia, Kateri, Ryan, Sally and Jyothi - graduate school has been so much more enjoyable because of their company. I especially would like to thank Mike, Anne and John for being there through the bad times and the good and making sure the good times were very good indeed! 


\section{Chapter 1}

\section{Introduction}

\section{The Semiclassical Idea}

The area of chemical dynamics aims at understanding physical processes at both the macroscopic and the microscopic levels. Classical mechanics (CM) has proven most effective in simulating the behavior of macroscopic systems; for smaller systems on the atomic and subatomic scale Quantum Mechanics (QM) provides the framework to understand and therefore predict various properties.

Semiclassical Theory (SC) has been around almost as long as Quantum Mechanics and for a very good reason. Historically, attempting to understand the new ideas in quantum mechanics was easiest apporached by finding classical analogues to quantum concepts. One of the earliest theories to do this was the Wentzel- 
Kramer-Brillouin ${ }^{1-3}$ theory (WKB), which we will discuss further a little later on.

We know that in some limits - large quantum number, high temperature, or in dynamic terms if the classical action is much larger than $\hbar$ - systems start to behave in a classical manner. In a realistic simulation this last mentioned asymptotic limit is reached quite easily. The action term that is the phase factor of the WKB wavefunction, for instance, has units of $\hbar$ and in the actual exponent appears as a ratio with $\hbar$. When this ratio is large, we see that the oscillations are very rapid and as a result most properties average out to classical behaviour. For instance, the smallest atom we simulate is the $\mathrm{H}$ atom. The action, as we know, is proportional to the square root of the mass. A H atom has a mass of about 1837 a.u. (atomic units), so the action will be about 40 times $\hbar(=1$ a.u.).

Keeping this in mind, the Semiclassical approach is, framed as an asymptotic limit theory and as such is most applicable when the action is several times greater than $\hbar$. We keep in mind that while there is a classical limit SC and a quantum limit SC, taking these limits is not identical to using either individual treatments - the theory is thus complete in itself and not just a special case.

The SC approach to dynamics provides us with the tools that not only elucidate the mechanics underlying chemical reactions but also provides a practical approach to 
simulating systems where quantum phenomena such as coherence,tunneling and zero point energy are of importance. Tbe basic idea is, then, to try and capture quantum effects using classical correspondence principles. By starting with a wavefunction with an amplitude and a phase factor(action) and by classically time evolving these components, we can obtain the information that when interpreted through the sufficiently non-classical structure of our formalism describes quantum effects.

\section{I.1 The WKB Theory}

The WKB theory is an early SC theory; a wavefunction with an amplitude and a phase factor that can be expanded in $\hbar$ to various orders is defined. By solving the Schrodinger equation for this wavefunction and using a slowly-varying phase approximation (which would correspond to the classical limit) we can characterize the phase and amplitude factors as well as obtaining a quantization condition. The WKB wavefunction expression is given by

$$
\psi(x) \propto \sum_{ \pm} \frac{1}{\sqrt{p(x)}} e^{ \pm i S(x) / \hbar} \cdot e^{ \pm i \pi / 4}
$$

The \pm sign under the summation limit indicates that we are in fact counting trajectories that propagate in either direction - tihs means the momentum can be positive or negative. The momentum $p(x)$ in the Eq. (1.1) is ,

$$
p(x)=\sqrt{\frac{2 m}{\hbar^{2}}(E-V(x))}
$$


and the action exponent is given by

$$
S(x, E)=\int^{x} p\left(x^{\prime}\right) d x^{\prime}
$$

The boundary condition here is that the wavefunction exponentially decays to zero in the classically forbidden region. We note that the momentum $p(x)$ is imaginary in these regions, thereby causing the wavefunction to go from being oscillatory to being decaying.

Imposing these boundary conditions leads to the quantization of energy levels. This is the Bohr Sommerfeld quantization condition ${ }^{4}$ and takes the form

$$
\left(n+\frac{1}{2}\right) \pi \hbar=\int_{x_{1}}^{x_{2}} p(x) d x
$$

where the $x_{1}$ and $x_{2}$ are the classical turning points which separate the classically 'allowed' and 'forbidden' regions. This equation can be inverted to find the eigenvalues.

The multidimensional equivalent of Eq. (1.1) is given by

$$
\psi(x)=\sum_{j}\left|\frac{\partial^{2} S(\mathbf{q}, E)}{\partial \mathbf{q} \partial E}\right|^{\frac{1}{2}} e^{i S(\mathbf{q}, E) / \hbar} e^{i \pi / 4}
$$

The derivation is described in some detail in a review paper by Berry et al. ${ }^{5}$ The corresponding multidimensional version ${ }^{6}$ of the quantization condition has also been obtained. In this case, a quantum state can be labelled by a vector quantum number

$$
n=\left(n_{1}, n_{2}, \ldots, n_{N}\right)
$$


This state comprises of the phase space trajectories of the corresponding classical system that lie on an $\mathrm{N}$-dimensional invariant toroid with a constant action $I_{k}$; the action here is part of the set of action-angle variables usesd to describe the system.

$$
I_{k}=\left(n_{k}+\text { constant }\right) \pi \hbar
$$

This quantization is referred to as the Einstein-Brillouin-Keller(EBK) condition. ${ }^{7-9}$

\section{I.2 The Van Vleck Propagator}

The Quantum mechanical time propagator $e^{-i \hat{H} t / \hbar}$ describes the evolution in time of wavefunctions (in the Schrödinger picture) or the operators (in the Heisenberg picture). The Semiclassical study of dynamics thus begins with crafting a classicallimit equivalent of this propagator. Van-Vleck ${ }^{10}$ first proposed the form this might take, based on his studies of the free particle propagator and the correspondence principle. This was developed further ${ }^{11}$ and can be derived as the stationary phase approximation to the Feynamn Path Integral. ${ }^{12}$

$$
\left\langle\mathbf{q}\left|e^{-i \hat{H} t / \hbar}\right| \mathbf{q}^{\prime}\right\rangle=(2 \pi i \hbar)^{-N / 2} \sum_{j}\left|\operatorname{Det}\left(\frac{\partial \mathbf{q}_{\mathbf{t}}}{\partial \mathbf{p}_{\mathbf{0}}}\right)\right|^{\frac{1}{2}} e^{i S_{j}\left(\mathbf{q}, \mathbf{q}^{\prime}, t\right) / \hbar} e^{-i \pi \nu_{j} / 2}
$$

The prefactor term here is introduced as the second derivative of action, $-\frac{\partial^{2} S_{j}}{\partial \mathbf{q} \partial \mathbf{q}^{\prime}}$ which then reduces to the monodromy matrix $\mathbf{M}_{q p}$ in the equation. The Maslov index $\nu_{j}$ ensures that we pick the right branch of the square root of the prefactor. The physical significance of this index was first described by Maslov ${ }^{13,14}$ as the 
equivalent of the WKB phase acquired across a classical turning point, arising in this case from an encounter with a caustic point ${ }^{15}$ in phase space.

The correspondence between classical and quantum mechanics can be seen in the parallel between classical canonical transformations and unitary transformations in quantum mechanics. The formalism for the propagator in Eq. (1.8) has also been derived by Miller, ${ }^{16}$ from the short time result of a canonical transformation from old variables $(\mathbf{p}, \mathbf{q})$ at time $t_{1}$ to new variables $(\mathbf{P}, \mathbf{Q})$ at time $t_{2}$; this can then be extended to a general classical limit expression for matrix elements of the propagator.

\section{I.3 Gaussian Wavepackets}

The Time-Dependent Schrödinger equation has been solved approximately with a wide variety of Gaussian wavepacket approaches. ${ }^{17}$ One of the first approximations was the 'Thawed' Gaussian approximation. ${ }^{18}$ The Ehrenfest theorem tells us that a Gaussian wavepacket in a harmonic potential will execute periodic motion while remaining Gaussian; and that the average position and momentum of the wavepacket follow the classical equations of motion.

It is assumed that with a sufficiently narrow wavepacket and a smooth potential, the space restriction imposed on the wavepacket will ensure that for the most part the Gaussian will see only the first few terms of a Taylor expansion in potential 
about the center of the wavepacket. The Gaussian wavepacket is written as

$$
\psi_{t}(q)=\left(\frac{R e\left[\alpha_{t}\right]}{\pi \hbar}\right)^{1 / 4} e^{-\frac{\alpha}{2 \hbar}\left(q-q_{t}\right)^{2}+\frac{i}{\hbar} p_{t}\left(q-q_{t}\right)+\frac{i}{\hbar} \beta_{t}}
$$

The parameters for the Gaussian are allowed to be time dependent; in this equation, $\alpha_{t}$ is complex and $\beta_{t}$ is real. The average position and momentum here are $q_{t}$ and $p_{t}$ respectively. The potential is expanded to second order around the center of the wavepacket

$$
V(q) \approx V_{0}+V_{q}\left(q-q_{t}\right)+\frac{V_{q q}}{2}\left(q-q_{t}\right)^{2}
$$

where $V_{q}$ and $V_{q q}$ are the first and second derivatives of the potential with respect to position.

The Frozen Gaussian Approximation ${ }^{19}$ was introduced to deal with the non-linear dependence of $(p, q)$ at time $t$ on the initial conditions of the trajectory that starts to creep in with longer time simulations. This is treated by expanding the wavepacket in terms of other gaussians, each centered at a slightly different position and momentum, so that the volume of phase space around the center of the individual gaussians where the linear assumption needs to be accurate is minimized.

$$
\psi_{t}(x)=\left(\frac{\omega}{\pi \hbar}\right)^{1 / 4} \sum_{j} c_{j} e^{-\frac{\omega}{2 \hbar}\left(q-q_{t j}\right)^{2}+\frac{i}{\hbar} p_{t j}\left(q-q_{t j}\right)+\frac{i}{\hbar} \gamma_{t j}}
$$

Neither the FGA nor the TGA require evaluation of the Maslov index or the prefactor term in Eq. (1.8). The 'derivation' of these methods is heuristic rather 
than rigorous.

One last wavepacket method we will mention here is the so-called Cellular approach to implement the Van Vleck propagator. This method involves Green's function propagation of an initial wavefunction from an ensemble of real trajectories without a root search.

\section{Thesis Outline}

This thesis is aimed at developing methods to accurately study the dynamics of complex systems. In particular we develop some methodology to make the Semiclassical Initial Value Representation (SC-IVR) easily extendible to large systems. We also tackle problems that are typical of complex systems where quantum effects become important along with ways to deal with nonadiabatic dynamics and accessing long time dynamics.

We start by describing the SC-IVR and several alternate/approximate forms of the IVR that have been developed over the years to tackle a variety of situations, making careful note of the advantages and disadvantages of each. Chapter 2 is devoted to a review of these existing methods. In Chapter 3 we describe a correction term for the energy of a system that can bring any approximate Quantum result closer to the exact value using Semiclassical Green's functions. Ideally this method will allow us to accurately determine, for instance, the vibrational energy 
levels of a polyatomic systems.

In Chapter 4, we examine the role of Monte Carlo methods that are used to evaluate the IVR propagator and how our choice of a sampling function makes a difference. We propose an algorithm to use time dependent sampling efficiently to improve our ability to capture long time dynamics while simultaneously reducing the number of trajectories required to reach statistical convergence. In Chapter 5, We use a classical electron model to simulate nonadiabatic dynamics by putting both the nuclear and electronic coordinates on the same footing. We also compare the various forms of IVR and rate their relative success.

Chapter 6 is about developing a tuning methodology that allows us to smoothly transition between the more classical forms of the SC-IVR and the more exact or 'quantum' limit of the SC propagator. The freedom to gradually turn on and off quantum effects for every individual degree of freedom in a large system will make simulating complex behaviour much simpler.

Finally, in Chapter 7, we describe an ongoing investigation into ways to simplify calculating the prefactor and tracking the Maslov index. The Hessian is required by most IVR methods in order to time evolve the Monodromy matrices that appear in the prefactor. We describe a finite difference scheme in time to calculate this Hessian on-the-fly so as to make the simulation as simple as any regular Molecular Dynamic (MD) simulation. Chapter 8 , briefly describes our various results and a concise summary is made of our attempts to scale the heights of quantum 
dynamical behaviour in complex systems. 


\section{Chapter 2}

\section{The Semiclassical Initial Value}

\section{Representation(SC-IVR)}

\section{Introduction}

This thesis is entirely devoted to the SC-IVR - applying it to different problems; extending and building on its framework to further its uses; and making its implementation as efficient as possible. In this chapter, we attempt to cover most of the existing literature on the IVR in all its various forms. Most of those described here will be referred to over and over again in the rest of this thesis; some IVR methodology that has not been directly used in the work done here has been omitted for instance, the very elegant time averaging method. A comprehensive overview of all the work related to the IVR can be found either in a concise review paper 
highlighting the work done in the Miller group specifically ${ }^{20}$ or a more general overview by Thoss and Wang ${ }^{21}$

Like the cellular approach described in the previous chapter the SC-IVR was developed as a way to get around the root search problem; the problem arises from having to identify the initial momentum for a classical trajectory that will enable it to reach a given final positions in the allotted time starting from a fixed initial position. This is a non-linear boundary value problem and computationally this can be done by using shooting algorithm where several trajectories with different momenta are generated for each initial position and only the ones which reach the appropriate final position in the given time are chosen to contribute to the integrand. This is a tedious procedure involving a lot of wasted effort in terms of the trajectories that are not used.

The original idea of carrying out a canonical transformation to change the variable of integration from final position to initial momentum within the integral over the initial position was proposed in the context of the propagator by Miller. ${ }^{22}$ A similar idea was proposed as a way obtain the S-Matrix semiclassically soon after. $^{23}$ More recently several groups have been working on using and extending this formalism ${ }^{20,24-30}$ 


\section{The IVR Trick}

In this section we illustrate the IVR idea as applied to the time propagator.

The matrix elements of the propagator between two states is a measure of the probability of a transition between these states.

Consider the transition amplitude between some initial state $\left|\psi_{i}\right\rangle$ and some final state $\left|\psi_{f}\right\rangle$.

$$
\begin{aligned}
\left\langle\psi_{f}\left|e^{-i \hat{H} t / \hbar}\right| \psi_{i}\right\rangle= & \sum_{\text {traj }} \int d \mathbf{q}_{\mathbf{0}} \int d \mathbf{q}_{\mathbf{t}}\left(\frac{1}{2 \pi i \hbar}\right)^{N / 2}\left\langle\psi_{f} \mid \mathbf{q}_{\mathbf{t}}\right\rangle \\
& \left|\operatorname{det}\left(\frac{\partial \mathbf{q}_{\mathbf{t}}}{\partial \mathbf{p}_{\mathbf{0}}}\right)\right|^{-\frac{1}{2}} e^{i S_{t}\left(\mathbf{q}_{\mathbf{t}}, \mathbf{q}_{\mathbf{0}}\right) / \hbar} e^{-i \pi \nu / 2}\left\langle\mathbf{q}_{\mathbf{0}} \mid \psi_{i}\right\rangle
\end{aligned}
$$

As described before, the sum is over all trajectories that start at position $\mathbf{q}_{\mathbf{0}}$ and end at position $\mathbf{q}_{\mathbf{t}}$ in time $t$. We need to find the set of all possible initial momenta $\mathbf{p}_{\mathbf{0}}$ that satisfy the condition

$$
\mathbf{q}_{\mathbf{t}}\left(\mathbf{q}_{0}, \mathbf{p}_{\mathbf{0}}\right)=\mathbf{q}_{\mathbf{t}}
$$

In general, there can be multiple roots for such a problem as show in figure Figure 2.1. The trick now, is to transform the integration over $q_{t}$ to one over $p_{0}$, within the $q_{0}$ integral. The $1 \mathrm{D}$ case is shown here for ease of illustration, but this is valid when the quantities involved are vectors too.

$$
\sum_{\text {roots }} \int d q_{t}=\int d p_{0}\left|\frac{\partial q_{t}\left(q_{0}, p_{0}\right)}{\partial p_{0}}\right|
$$




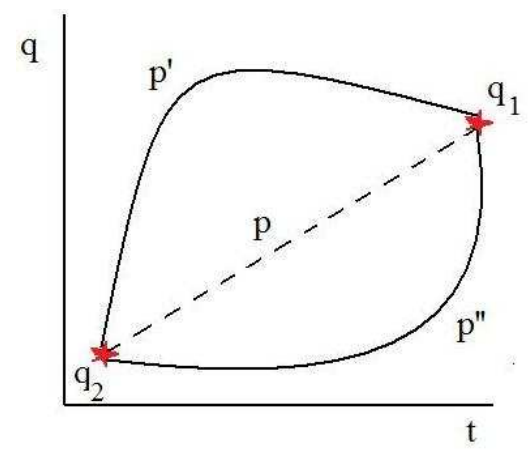

Figure 2.1: Non-linear boundary value problem

The Jacobian comes from the change of variable. Figure 2.2 also shows us quite clearly that integrating over all possible values of $q_{t}$ and adding up the separate branches is equivalent to integrating over all $p_{0}$. The expression for the transition

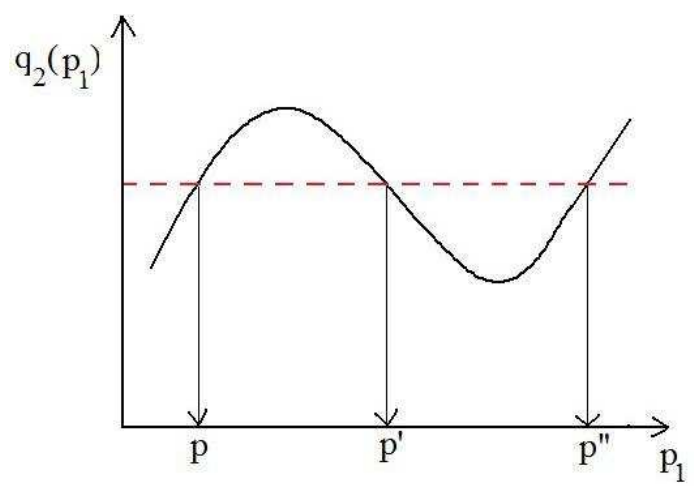

Figure 2.2: Showing the different branches of the function $q_{2}\left(p_{1}\right)$ 
amplitude can now be written as,

$$
\begin{aligned}
\left\langle\psi_{f}\left|e^{-i \hat{H} t / \hbar}\right| \psi_{i}\right\rangle= & \left(\frac{1}{2 \pi i \hbar}\right)^{N / 2} \int d \mathbf{q}_{\mathbf{0}} \int d \mathbf{p}_{\mathbf{0}}\left\langle\psi_{f} \mid \mathbf{q}_{\mathbf{t}}\right\rangle \\
& \mid \operatorname{det}\left(\frac{\partial \mathbf{q}_{\mathbf{t}}}{\partial \mathbf{p}_{\mathbf{0}}}\right)^{\frac{1}{2}} e^{i S_{t}\left(\mathbf{q}_{\mathbf{0}}, \mathbf{p}_{\mathbf{0}}\right) / \hbar} e^{-i \pi \nu / 2}\left\langle\mathbf{q}_{\mathbf{0}} \mid \psi_{i}\right\rangle
\end{aligned}
$$

The expression Eq. (2.4) is merely a variable-changed version of the original Eq. (2.1). It is, therefore, just as exact with a couple of practical advantages. First, the integration is now over the phase space of initial conditions. This space can be effectively sampled by Monte Carlo methods and the classical trajectories drawn from this space are uniquely determined. Second, the Jacobian term in the transformation in Eq. (2.3) is now in the numerator of our final expression as opposed to in the denominator as in Eq. (1.8). This means that the zeroes that the Jacobian may go through no longer cause singularities.

\section{II.1 Herman Kluk IVR (HK-IVR)}

The IVR expression in Eq. (2.4) has the disadvantage that there is no obvious sampling function for the momentum integral. It is possible to derive a similar expression with initial and final momentum states, but this leaves us with no position sampling function. Most wavepackets are localized only in one or the other of position and momentum. Coherent states $^{31}$ are uniquely useful in that they minimize the product of the uncertainties in position and momentum (hence the name minimum uncertainty wavepackets). In other words, coherent states are localized 
in both position and momentum.

Exploiting this fact in the Herman Kluk IVR (HK-IVR), ${ }^{32}$ an expression for the time propagator resembling Eq. (2.4) is obtained for coherent states. The original derivation was based on the FGA idea, but instead of expanding the initial wavefunction as a sum of gaussian wavepackets, Herman and Kluk used the overcomplete set of coherent states.

$\left\langle\psi_{f}\left|e^{-i \hat{H} t / \hbar}\right| \psi_{i}\right\rangle=\left(\frac{1}{2 \pi \hbar}\right)^{N / 2} \int d \mathbf{q}_{\mathbf{0}} d \mathbf{p}_{\mathbf{0}}\left\langle\psi_{f} \mid \mathbf{p}_{\mathbf{t}}, \mathbf{q}_{\mathbf{t}}\right\rangle C_{t}\left(\mathbf{p}_{\mathbf{0}}, \mathbf{q}_{\mathbf{0}}\right) e^{i S_{t}\left(\mathbf{p}_{\mathbf{0}}, \mathbf{q}_{\mathbf{0}}\right) / \hbar}\left\langle\mathbf{p}_{\mathbf{0}}, \mathbf{q}_{\mathbf{0}} \mid \psi_{i}\right\rangle$

The coordinate space wavefunctions of the coherent states are give by

$$
\langle\mathbf{x} \mid \mathbf{p}, \mathbf{q}\rangle=\Pi_{j=1}^{N}\left(\frac{\gamma_{j}}{\pi}\right)^{1 / 4} e^{-\frac{\gamma_{j}}{2}\left(x_{j}-q_{j}\right)^{2}} e^{i p_{j}\left(x_{j}-q_{j}\right)} / \hbar
$$

The pre-exponential factor $C_{t}$ is given by

$$
\begin{aligned}
& C_{t}\left(\mathbf{p}_{\mathbf{0}}, \mathbf{q}_{\mathbf{0}}\right)= \\
& \operatorname{det}\left[\frac{1}{2}\left(\gamma^{\frac{1}{2}} \frac{\partial \mathbf{q}_{\mathbf{t}}}{\partial \mathbf{q}_{\mathbf{0}}} \gamma^{-\frac{1}{2}}+\gamma^{-\frac{1}{2}} \frac{\partial \mathbf{p}_{\mathbf{t}}}{\partial \mathbf{p}_{\mathbf{0}}} \gamma^{\frac{1}{2}}-i \hbar \gamma^{\frac{1}{2}} \frac{\partial \mathbf{q}_{\mathbf{t}}}{\partial \mathbf{p}_{\mathbf{0}}} \gamma^{\frac{1}{2}}+\frac{i}{\hbar} \gamma^{-\frac{1}{2}} \frac{\partial \mathbf{p}_{\mathbf{t}}}{\partial \mathbf{q}_{\mathbf{0}}} \gamma^{-\frac{1}{2}}\right)\right]^{\frac{1}{2}}
\end{aligned}
$$

The HK-IVR formulation is further generalized where the coherent state width $\gamma$ is different for the initial state $\left|\mathbf{p}_{\mathbf{0}}, \mathbf{q}_{\mathbf{0}}\right\rangle$ and the final state $\left|\mathbf{p}_{\mathbf{t}}, \mathbf{q}_{\mathbf{t}}\right\rangle{ }^{33}$ In the limit of $\gamma \rightarrow \infty$ the coherent states in Eq. (2.5) reduce to position states and to momentum states in the limit $\gamma \rightarrow 0$.

There is some debate as to the exact-ness of this HK-IVR. It has been showed that Filinov smoothing (described later in this manuscript) applied to the Van-Vleck 
IVR Eq. (1.8) actually produces this exact formalism; ${ }^{34}$ this leads us to believe that the HK-IVR propagator is non-exact. The many methods to derive the HKIVR are reviewed in a somewhat recent article by Deshpande et al. ${ }^{35}$

However, expressing the propagator by Eq. (2.5) has proved to be very useful in several practical problems, because this formalism provides us with a good sampling function in the form of the overlap $\left\langle\mathbf{p}_{\mathbf{0}}, \mathbf{q}_{\mathbf{0}} \mid \psi_{i}\right\rangle$ for both the position and momentum integrals. In practice, we use the square of this function as the sampling function, because this takes the form of a convenient, normalizable gaussian function - this is the Husimi distribution.

\section{Correlation Functions}

Time Correlation functions prove to be very useful theoretical tools for the calculation of several physical properties observed spectroscopically or by other measurements.

$$
C_{A B}(t)=\left[e^{-\beta \hat{H}} \hat{A} e^{i \hat{H} t / \hbar} \hat{B} e^{-i \hat{H} t / \hbar}\right]
$$

Operators $\hat{A}$ and $\hat{B}$ are chosen depending on the property we wish to study. The dipole-dipole correlation function, for instance, is obtained by setting $\hat{A}=\hat{B}=\hat{\mu}$ where $\mu$ is the transition dipole operator. The Fourier transform of this correlation function gives us the electronic absorption spectrum. Similarly, the time integral of 
the flux-flux correlation function gives the rate of a chemical reaction. The ability to evaluate correlation functions like in Eq. (3.8) is, therefore central to any type of dynamical method.

\section{III.1 Double HK-IVR (DHK-IVR)}

Using IVR, the simplest way to evaluate a correlation function is to substitute, say, the HK-IVR expression Eq. (2.5) for the two propagators in Eq. (3.8).

$$
\begin{aligned}
& C_{A B}(t)=(2 \pi \hbar)^{-2 F} \int d \mathbf{q}_{0} \int d \mathbf{p}_{0} \int d \mathbf{q}_{t}^{\prime} \int d \mathbf{p}_{t}^{\prime} \mathcal{C}_{-t}\left(\mathbf{q}_{t}^{\prime}, \mathbf{p}_{t}^{\prime} ; \gamma_{o}^{\prime}, \gamma_{t}^{\prime}\right) \mathcal{C}_{t}\left(\mathbf{q}_{0}, \mathbf{p}_{0} ; \gamma_{o}, \gamma_{t}\right) \\
&\left\langle\mathbf{p}_{t}^{\prime}, \mathbf{q}_{t}^{\prime} ; \gamma_{t}^{\prime}|\hat{B}| \mathbf{p}_{t}, \mathbf{q}_{t} ; \gamma_{t}\right\rangle e^{\frac{i}{\hbar} S_{t}\left(q_{0}, p_{0}\right)} e^{\frac{i}{\hbar} S_{-t}\left(\mathbf{q}_{t}^{\prime}, \mathbf{p}_{t}^{\prime}\right)} \\
&\left\langle\mathbf{p}_{0}, \mathbf{q}_{0} ; \gamma_{o}|\hat{A}| \mathbf{p}_{0}^{\prime}, \mathbf{q}_{0}^{\prime} ; \gamma_{o}^{\prime}\right\rangle
\end{aligned}
$$

where we have used the most general form of the HK-IVR possible, with different $\gamma$ values for each of the coherent states.

The trajectories begin at phase space point $\left(\mathbf{p}_{\mathbf{0}}, \mathbf{q}_{\mathbf{0}}\right)$ and undergo forward time evolution to the point $\left(\mathbf{p}_{\mathbf{t}}, \mathbf{q}_{\mathbf{t}}\right)$. At time $t$ there is a 'jump' in both position and momentum

$$
\begin{aligned}
& \mathrm{p}_{\mathrm{t}}^{\prime}=\mathrm{p}_{\mathrm{t}}+\Delta \mathrm{p} \\
& \mathrm{q}_{\mathrm{t}}^{\prime}=\mathrm{q}_{\mathrm{t}}+\Delta \mathrm{q}
\end{aligned}
$$

This jump is followed by a backward time evolution from initial point $\left(\mathbf{p}_{\mathbf{t}}^{\prime}, \mathbf{q}_{\mathbf{t}}^{\prime}\right)$, also for a time $t$, to $\left(\mathbf{p}_{\mathbf{0}}^{\prime}, \mathbf{q}_{\mathbf{0}}^{\prime}\right)$. The path of the classical trajectories needed to evaluate 
this expression is shown in Figure 2.3.

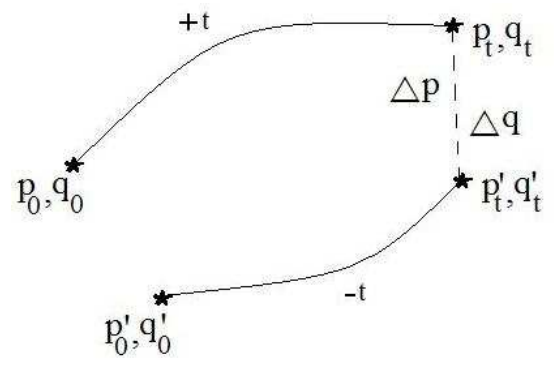

Figure 2.3: Schematic representation of the path described by the classical trajectory in our simulation.

The prefactor for the forward trajectory is the same as the HK-IVR in Eq. (2.7); In this case we write out the most general form with different coherent state widths

$$
\begin{aligned}
& C_{t}\left(\mathbf{p}_{\mathbf{0}}, \mathbf{q}_{\mathbf{0}}\right)= \\
& \operatorname{det}\left[\frac{1}{2}\left(\gamma_{t}^{\frac{1}{2}} \frac{\partial \mathbf{q}_{\mathbf{t}}}{\partial \mathbf{q}_{\mathbf{0}}} \gamma_{0}^{-\frac{1}{2}}+\gamma_{t}^{-\frac{1}{2}} \frac{\partial \mathbf{p}_{\mathbf{t}}}{\partial \mathbf{p}_{\mathbf{0}}} \gamma_{0}^{\frac{1}{2}}-i \hbar \gamma_{t}^{\frac{1}{2}} \frac{\partial \mathbf{q}_{\mathbf{t}}}{\partial \mathbf{p}_{\mathbf{0}}} \gamma_{0}^{\frac{1}{2}}+\frac{i}{\hbar} \gamma_{t}^{-\frac{1}{2}} \frac{\partial \mathbf{p}_{\mathbf{t}}}{\partial \mathbf{q}_{\mathbf{0}}} \gamma_{0}^{-\frac{1}{2}}\right)\right]^{\frac{1}{2}}
\end{aligned}
$$

The prefactor for the backward trajectory is,

$$
\begin{aligned}
& C_{-t}\left(\mathbf{p}_{\mathbf{t}}^{\prime}, \mathbf{q}_{\mathbf{t}}^{\prime}\right)= \\
& \operatorname{det}\left[\frac{1}{2}\left(\gamma_{0}{ }^{\frac{1}{2}} \frac{\partial \mathbf{q}_{\mathbf{0}}^{\prime}}{\partial \mathbf{q}_{\mathbf{t}}^{\prime}} \gamma_{t^{\prime}}{ }^{\frac{1}{2}}+\gamma_{0} \prime^{-\frac{1}{2}} \frac{\partial \mathbf{p}_{\mathbf{0}}^{\prime}}{\partial \mathbf{p}_{\mathbf{t}}^{\prime}} \gamma_{t^{\frac{1}{2}}}-i \hbar \gamma_{0} \prime^{\frac{1}{2}} \frac{\partial \mathbf{q}_{\mathbf{0}}^{\prime}}{\partial \mathbf{p}_{\mathbf{t}}^{\prime}} \gamma_{t^{\prime}} \frac{1}{\hbar}+\frac{i}{\hbar} \gamma_{0} \prime^{-\frac{1}{2}} \frac{\partial \mathbf{p}_{\mathbf{0}}^{\prime}}{\partial \mathbf{q}_{\mathbf{t}}^{\prime}} \gamma_{t^{\prime}}{ }^{\frac{1}{2}}\right)\right]^{\frac{1}{2}}
\end{aligned}
$$

The expression in Eq. (3.9) is referred to as the Double Herman-Kluk IVR (DHKIVR) and not only involves a double phase space Monte Carlo, but also a potentially very oscillatory integrand. The advantages of this formalism are two-fold. First and most importantly, the DHK-IVR is semiclassically exact. (We qualify this statement by saying its as exact as the HK-IVR is taken to be!) Second, in 
many cases we find that we can write

$$
\left\langle\mathbf{p}_{\mathbf{t}}^{\prime}, \mathbf{q}_{\mathbf{t}}^{\prime}|\hat{B}| \mathbf{p}_{\mathbf{t}}, \mathbf{q}_{\mathbf{t}}\right\rangle=F\left(\mathbf{q}_{\mathbf{t}}^{\prime}, \mathbf{p}_{\mathbf{t}}^{\prime}, \mathbf{q}_{\mathbf{t}}, \mathbf{p}_{\mathbf{t}}\right)\left\langle\mathbf{p}_{\mathbf{t}}^{\prime}, \mathbf{q}_{\mathbf{t}}^{\prime} \mid \mathbf{p}_{\mathbf{t}}, \mathbf{q}_{\mathbf{t}}\right\rangle
$$

This allows us to use the overlap function of the two coherent states at time $t$ as sampling function for the jump since

$$
\left\langle\mathbf{p}_{\mathbf{t}}^{\prime}, \mathbf{q}_{\mathbf{t}}^{\prime} \mid \mathbf{p}_{\mathbf{t}}, \mathbf{q}_{\mathbf{t}}\right\rangle=e^{-\frac{\gamma_{t}}{4}\left(\mathbf{q}_{\mathbf{t}}^{\prime}-\mathbf{q}_{\mathbf{t}}\right)^{2}} e^{-\frac{1}{4} \gamma_{t}\left(\mathbf{p}_{\mathbf{t}}^{\prime}-\mathbf{p}_{\mathbf{t}}\right)^{2}} e^{\frac{i}{2}\left(\mathbf{p}_{\mathbf{t}}^{\prime}+\mathbf{p}_{\mathbf{t}}\right)\left(\mathbf{q}_{\mathbf{t}}^{\prime}-\mathbf{q}_{\mathbf{t}}\right)}
$$

The real exponents in this overlap are used to sample the Monte Carlo integration and the imaginary exponent is grouped in with the rest of the integrand.

There are several approximations that can be made to reduce the variables of integration as well as to smooth out the oscillatory nature of the integrand.

\section{III.2 Exact Forward-Backward IVR (EFB-IVR)}

One way to remove the need to evaluate a double phase space integral is the 'Exact' Forward-Backward IVR, as described in. ${ }^{36}$ It is so called, because this expression, like the Double HK-IVR, involves no approximation other than the basic IVR description of the propagator.

The EFB-IVR can be described most easily for a case where it is assumed that the

operator $\hat{B}$ is a function of either momentum operators only or position operators only. Here, we follow the derivation under the assumption that the operator is a function of only momentum operator(s).

$$
\hat{B}=B(\hat{\mathbf{p}})
$$


Consider the DHK-IVR expression described by Eq. (3.9) along with the description of the various terms as described in Eq. (3.12) and Eq. (3.13).

The momentum space matrix of operator $\hat{B}$ is diagonal (by assumption). This fact can best be used in evaluating the term $\left\langle\mathbf{p}_{\mathbf{t}}^{\prime}, \mathbf{q}_{\mathbf{t}}^{\prime}|\hat{B}| \mathbf{p}_{\mathbf{t}}, \mathbf{q}_{\mathbf{t}}\right\rangle$ if the coherent states are reduced to momentum states. As discussed previously, this can be done by taking the limit $\gamma_{t} \rightarrow 0$ and $\gamma_{t} \prime \rightarrow 0$

$$
\begin{aligned}
C_{A B}(t)= & (2 \pi \hbar)^{-F} \int d \mathbf{q}_{\mathbf{0}} \int d \mathbf{p}_{\mathbf{0}} \int d \mathbf{q}_{\mathbf{t}}^{\prime} \mathcal{D}_{t}^{f}\left(\mathbf{q}_{\mathbf{0}}, \mathbf{p}_{\mathbf{0}} ; \gamma_{0}\right) \mathcal{D}_{-t}^{b}\left(\mathbf{q}_{\mathbf{t}}^{\prime}, \mathbf{p}_{\mathbf{t}}, \gamma_{0} \prime\right) B\left(\mathbf{p}_{\mathbf{t}}\right) \\
& e^{\frac{i}{\hbar} S_{t}\left(q_{0}, p_{0}\right)} e^{\frac{i}{\hbar} S_{-t}\left(q_{t}^{\prime}, p_{t}\right)} e^{\frac{i}{\hbar} \mathbf{p}_{\mathbf{t}} \cdot\left(\mathbf{q}_{\mathbf{t}}^{\prime}-\mathbf{q}_{\mathbf{t}}\right)}\left\langle\mathbf{p}_{\mathbf{0}}, \mathbf{q}_{\mathbf{0}} ; \gamma_{0}|\hat{A}| \mathbf{p}_{\mathbf{0}}^{\prime}, \mathbf{q}_{\mathbf{0}}^{\prime} ; \gamma_{0} \prime\right\rangle
\end{aligned}
$$

The operator $\hat{B}$ acts upon the momentum states obtained in the limit to give us $B\left(\mathbf{p}_{\mathbf{t}}\right) \delta\left(\mathbf{p}_{\mathbf{t}}^{\prime}-\mathbf{p}_{\mathbf{t}}\right)$. The integral over $\mathbf{p}_{\mathbf{t}}^{\prime}$ can now be carried out, leaving us with only a integral over the position $\mathbf{q}_{\mathbf{t}}^{\prime}$. The forward and backward prefactors in this same limit take the form,

$$
\begin{aligned}
& \mathcal{D}_{t}^{f}\left(\mathbf{q}_{\mathbf{0}}, \mathbf{p}_{\mathbf{0}} ; \gamma_{0}\right)=\left|\frac{1}{2 \sqrt{\pi}}\left(\mathbf{M}_{p p}^{f} \gamma_{0}^{\frac{1}{2}}+\frac{i}{\hbar} \mathbf{M}_{p q}^{f} \gamma_{0}^{-\frac{1}{2}}\right)\right|^{1 / 2} \\
& \mathcal{D}_{-t}^{b}\left(\mathbf{q}_{t}^{\prime}, \mathbf{p}_{t}, \gamma_{o}^{\prime}\right)=\left|\frac{1}{2 \sqrt{\pi}}\left(\gamma_{0}^{\prime \frac{1}{2}} \mathbf{M}_{q q}^{b}+\frac{i}{\hbar} \gamma_{0} \prime^{-\frac{1}{2}} \mathbf{M}_{p q}^{b}\right)\right|^{1 / 2}
\end{aligned}
$$

The trajectory follows a very similar path to the one described for the DHK-IVR; the only difference being that there is no longer a momentum jump in any of the degrees of freedom at time $t$, only a position jump. One further note here is that we can sometimes replace the integral over $\mathbf{q}_{\mathbf{t}}^{\prime}$ in Eq. (3.17) by an integral over the position jump $\Delta \mathbf{q}=\mathbf{q}_{\mathbf{t}}^{\prime}-\mathbf{q}_{\mathbf{t}}$, a simple change of variable. 
The formulation for the case where operator $\hat{B}$ is a function of position only, can be derived in a similar fashion and the resulting expression will involve only a momentum jump in the trajectories. The prefactors are also modified suitably and in this case they are of the form

$$
\begin{aligned}
& \mathcal{D}_{t}^{f}\left(\mathbf{q}_{\mathbf{0}}, \mathbf{p}_{\mathbf{0}} ; \gamma_{0}\right)=\left|\frac{1}{2 \sqrt{\pi}}\left(\mathbf{M}_{q q}^{f} \gamma_{0}^{-\frac{1}{2}}-i \hbar \mathbf{M}_{q p}^{f} \gamma_{0}^{\frac{1}{2}}\right)\right|^{1 / 2} \\
& \mathcal{D}_{-t}^{b}\left(\mathbf{q}_{t}^{\prime}, \mathbf{p}_{t}, \gamma_{o}^{\prime}\right)=\left|\frac{1}{2 \sqrt{\pi}}\left(\gamma_{0} \prime^{-\frac{1}{2}} \mathbf{M}_{p p}^{b}-i \hbar \gamma_{0}{ }^{\frac{1}{2}} \mathbf{M}_{q p}^{b}\right)\right|^{1 / 2}
\end{aligned}
$$

While there is no need to evaluate a double phase space integral, it must be noted that in this formulation for the correlation function, we are left entirely without a way to sample the $\mathbf{q}_{\mathbf{t}}^{\prime}$. In some cases, this is not a problem, but for a larger system having to evaluate the multidimensional integral over all the jump degrees of freedom on a grid can be quite a challenge.

\section{III.3 The Forward Backward (FB-IVR)}

The FB-IVR first described elsewhere ${ }^{37,38}$ is an approximate method to describe correlation functions unlike the both the DHK-IVR and the EFB-IVR. Despite this, the FB-IVR is in fact the simplest way to obtain the correlation function while still capturing coherence effects.

As in the case of the EFB-IVR, operator $\hat{B}$ is chosen such that involves only momentum operator(s). The operator is assumed to be in the form of a local 
phase factor

$$
\hat{B}=e^{i \Phi(\hat{\mathbf{p}})}
$$

The overall operator involved in the correlation function of the form Eq. (3.8) can be written as

$$
\hat{U}=e^{i \hat{H} t / \hbar} e^{i \Phi(\hat{\mathbf{p}})} e^{-i \hat{H} t / \hbar}
$$

The operator $\hat{U}$ is unitary and can be treated as a single time evolution operator that goes forward to time $t$ and then backward from $t \rightarrow 0$ under a time dependent Hamiltonian of the form

$$
\hat{H}\left(t^{\prime}\right)= \begin{cases}\hat{H}-\delta\left(t-t^{\prime}\right) \Phi(\hat{p}) & 0 \rightarrow t \\ \hat{H} & t \rightarrow 0\end{cases}
$$

where $\hat{H}$ is the original time independent Hamiltonian.

The trajectory starts at $\left(\mathbf{p}_{\mathbf{0}}, \mathbf{q}_{\mathbf{0}}\right)$ and evolves forward in time under the time independent Hamiltonian to $\left(\mathbf{p}_{\mathbf{t}}, \mathbf{q}_{\mathbf{t}}\right)$. The momentum and position at time $t$ the evolve in time under the additional time dependent part of the Hamiltonian.

$$
\mathbf{p}_{\mathbf{t}}^{\prime} \rightarrow \mathbf{p}_{\mathbf{t}}+\left(\frac{\partial \Phi(\hat{\mathbf{p}})}{\partial \mathbf{q}}\right)_{\mathbf{q}=\mathbf{q}_{\mathbf{t}}}
$$

In this case, our choice of the form of operator $\hat{B}$ makes its derivative with respect to position zero, so we have no momentum jump, something this FB-IVR formulation has in common with the EFB-IVR. The position jump is also determined 
by the time dependent part of the Hamiltonian,

$$
\mathbf{q}_{\mathbf{t}}^{\prime} \rightarrow \mathbf{q}_{\mathbf{t}}-\left(\frac{\partial \Phi(\hat{\mathbf{p}})}{\partial \mathbf{p}}\right)_{\mathbf{p}=\mathbf{p}_{\mathbf{t}}}
$$

The trajectory is then evolved backward in time under the time independent Hamiltonian to $\left(\mathbf{p}_{\mathbf{0}}^{\prime}, \mathbf{q}_{\mathbf{0}}^{\prime}\right)$.

The IVR implementation of $\hat{U}$ (which is the time propagator here) is done using the standard propagator HK-IVR.

$$
\hat{U}=(2 \pi \hbar)^{-F} \int d \mathbf{p}_{\mathbf{0}} \int d \mathbf{q}_{\mathbf{0}} C_{0}\left(\mathbf{p}_{\mathbf{0}}, \mathbf{q}_{\mathbf{0}}\right) e^{i S_{0}\left(\mathbf{p}_{\mathbf{0}}, \mathbf{q}_{\mathbf{0}}\right) / \hbar}\left|\mathbf{p}_{\mathbf{0}}^{\prime}, \mathbf{q}_{\mathbf{0}}^{\prime}\right\rangle\left\langle\mathbf{p}_{\mathbf{0}}, \mathbf{q}_{\mathbf{0}}\right|
$$

The trajectory follows the path previously described. The action integral here is given by,

$$
\begin{aligned}
S_{0}\left(\mathbf{p}_{\mathbf{0}}, \mathbf{q}_{\mathbf{0}}\right) & =\int_{0}^{t} d t^{\prime}[\mathbf{p} \cdot \dot{\mathbf{q}}-H(\mathbf{p}, \mathbf{q})]+\Delta S \\
& +\int_{t}^{0} d t^{\prime}[\mathbf{p} \cdot \dot{\mathbf{q}}-H(\mathbf{p}, \mathbf{q})]
\end{aligned}
$$

where the additional action term is

$$
\Delta S=-\mathbf{p}_{t} \cdot \frac{\partial \Phi\left(\mathbf{p}_{t}\right)}{\partial \mathbf{p}_{t}}+\Phi\left(\mathbf{p}_{t}\right)
$$

If $\Phi(\hat{\mathbf{p}})$ is a linear function of momentum, the action add-on term vanishes.

The prefactor $C_{0}\left(\mathbf{p}_{\mathbf{0}}, \mathbf{q}_{\mathbf{0}}\right)$ is the same as the Herman-Kluk prefactor of Eq. (2.7) with the Monodromy matrices defined for the full trajectory such as,

$$
\mathbf{M}_{q q}=\frac{\partial \mathbf{q}_{\mathbf{0}}^{\prime}}{\partial \mathbf{q}_{\mathbf{0}}}
$$


The FB-IVR correlation function is therefore simply a single phase space average over initial conditions.

$$
C_{A B}(t)=(2 \pi \hbar)^{-F} \int d \mathbf{p}_{\mathbf{0}} \int d \mathbf{q}_{\mathbf{0}} C_{0}\left(\mathbf{p}_{\mathbf{0}}, \mathbf{q}_{\mathbf{0}}\right) e^{i S_{0}\left(\mathbf{p}_{\mathbf{0}}, \mathbf{q}_{\mathbf{0}}\right) / \hbar}\left\langle\mathbf{p}_{\mathbf{0}}, \mathbf{q}_{\mathbf{0}}|\hat{A}| \mathbf{p}_{\mathbf{0}}^{\prime}, \mathbf{q}_{\mathbf{0}}^{\prime}\right\rangle
$$

The FB-IVR can be shown to come from making a stationary phase approximation to the DHK-IVR and is an important result that reduces the double phase space average over initial conditions to a single such average. A number of applications have shown that the FB-IVR can indeed describe true quantum coherence effects

\section{III.4 LSC-IVR}

If one uses the coordinate space IVR, for the two propagators in the correlation function Eq. (3.8), and then approximates the integrand by expanding all quantities to first order in the difference of two sets of initial conditions, then the classical Wigner model ${ }^{39-41}$ is obtained to be

$$
C_{A B}(t)=(2 \pi \hbar)^{-F} \int d \mathbf{p}_{0} \int d \mathbf{q}_{0} A_{w}\left(\mathbf{p}_{0}, \mathbf{q}_{0}\right) B_{w}\left(\mathbf{p}_{t}, \mathbf{q}_{t}\right)
$$

where $A_{w}$ and $B_{w}$ are the Wigner functions corresponding to operators $\hat{A}$ and $\hat{B}$, respectively, e.g.

$$
A_{w}(\mathbf{p}, \mathbf{q})=\int d \mathbf{\Delta} \mathbf{q} e^{-i \mathbf{p}^{T} \cdot \Delta \mathbf{q} / \hbar}\left\langle\mathbf{q}+\frac{\Delta \mathbf{q}}{2}|\hat{A}| \mathbf{q}-\frac{\Delta \mathbf{q}}{2}\right\rangle
$$

This linearized SC-IVR (LSC-IVR)/classical Wigner model is an old idea and has been around for a long time; the interest here is seeing that it is contained within 
the SC-IVR, being a (rather crude) approximation to it. (And there are even other, more recent derivations of the Wigner model from other starting points). ${ }^{42,43}$ One sees that it has precisely the form of the classical time correlation function, only with the classical functions corresponding to operators $\hat{A}$ and $\hat{B}$ replaced by their Wigner functions. 


\section{Chapter 3}

\section{A Semiclassical Correction Term}

\section{for Energy Eigenvalues}

\section{Introduction}

Establishing the energy spectrum of large molecules is the subject of much work in theoretical chemistry. Exact quantum mechanical methods can be used only for systems with very few degrees of freedom as the cost of the calculation scales exponentially with system size. Several approximate quantum methods have therefore, been developed to tackle this problem. Many of these methods, like the SelfConsistent Field (SCF) method, are either based on the Variational principle or some kind of Perturbation theory to different orders. Other approximate methods to calculate the energy spectrum include semiclassical methods, such as the 
Semiclassical Initial Value Representation (SC-IVR) previously introduced; and yet other approaches that do not invoke the propagator at all and are more along the lines of a WKB approximation. These methods have been shown to be quite effective in obtaining the energy spectrum. ${ }^{44,45}$ In this chapter we describe a method to best use the SC-IVR to obtain accurate energy spectra.

One common way to obtain the energy spectrum is by exploiting the relationship between the spectral density function and the time propagator. The formal representation of the spectral density in Quantum Mechanics has delta function peaks corresponding to the eigenvalues of the Hamiltonian. If we have an eigenvalue equation

$$
\hat{H}\left|\phi_{i}\right\rangle=E_{i}\left|\phi_{i}\right\rangle
$$

then the spectral density is

$$
\rho(E)=\sum_{i} \delta\left(E-E_{i}\right)\left|\left\langle\phi_{i} \mid \Psi\right\rangle\right|^{2}
$$

where the strength of the delta function peak is weighted by the overlap of the chosen reference state with the eigenkets $\left|\phi_{i}\right\rangle$.

We can also express the spectral density as a trace,

$$
\rho(E)=\langle\Psi|\delta(E-\hat{H})| \Psi\rangle
$$

where $|\Psi\rangle$ is just any reference state.

This delta function (the microcanonical density operator) is related to the real 
time propagator by,

$$
\delta(E-\hat{H})=\frac{R e}{\pi \hbar} \int_{0}^{\infty} d t e^{i E t / \hbar} e^{-i \hat{H} t / \hbar}
$$

Combining Eq. (1.4) and Eq. (1.3), we see that the spectral density function $\rho(E)$ is the half Fourier transform of the survival amplitude $\left\langle\Psi\left|e^{-i \hat{H} t / \hbar}\right| \Psi\right\rangle$

The time propagator in Eq. (1.4) can be evaluated by exact quantum mechanical techniques such as the split-operator method, or the Chebyshev method ${ }^{46}$ for a limited system size.

Intuitively, however, given a choice, we would (and quite rightly!) pick to use an approximate quantum method over a semiclassical one. With this in mind, we propose a two step process to evaluate energy spectra. As a first step, a QM approximation can be used to obtain a good estimate of the energies. In the second step, a correction term that take the existing good estimate and makes it better is evaluated with semiclassical methods. A closer look at what such a correction term can accomplish is quite encouraging. Consider a variational quantum approach such as the Self-Consistent Field method(SCF). The correction term in this case corresponds to the correlation energy. If this correlation energy is about $10 \%$ of the total energy and we assume that the semiclassical calculation captures up to $90 \%$ of this correction term then the accuracy of the overall result has now been increased to about $1 \%$. 
The idea of such a Semiclassical correction term has been used before, ${ }^{47}$ in the context of improving on a Kohn variational calculation for the S-matrix. We will briefly review this in the Methods section before going on to describe our analogous approach to obtain an energy correction term. An important point here is that the correction term is formulated such that the total energy expression is formally exact and the only approximation introduced is in its evaluation by semiclassical methods.

\section{Theory}

\section{II.1 SC correction to the S-Matrix}

The S-matrix can be written using the Distorted Wave Born Approximation (see, for instance, see Merzbacher ${ }^{48}$ ) as

$$
\begin{aligned}
S(E) & =S_{0}(E)+\frac{i}{\hbar}\left[\langle\phi|\hat{W}| \phi\rangle+\left\langle\phi\left|\hat{W} \hat{G}_{0}^{+} \hat{W}\right| \phi\right\rangle\right. \\
& \left.+\left\langle\phi\left|\hat{W} \hat{G}_{0}^{+} \hat{W} \hat{G}_{0}^{+} \hat{W}\right| \phi\right\rangle+\ldots\right]
\end{aligned}
$$

We can separate the Hamiltonian into a so-called 'Distorted Wave' (DW) Hamiltonian $\left(\hat{H}_{0}\right)$ and a residual interaction term $(\hat{W}) . \phi(r)$ is the scattering solution at energy E

$$
\left(E-\hat{H}_{0}\right)|\phi\rangle=0
$$


The boundary condition for this solution is that in the asymptotic limit the function takes the form

$$
\phi(r)=-\frac{e^{-i k r}}{v^{\frac{1}{2}}}+\frac{e^{i k r}}{v^{\frac{1}{2}}} S_{0}
$$

where, $v=\frac{\hbar k}{\mu}$ is the asymptotic velocity and $\mu$ is the reduced mass.

$S_{0}(E)$ corresponds to this DW solution and the DW Green's function is

$$
\hat{G}_{0}=\frac{1}{E-\hat{H}_{0}+i \epsilon}
$$

The formal sum of the DW Born Series is well known and gives us

$$
\hat{G}_{0}^{+}+\hat{G}_{0}^{+} \hat{W} \hat{G}_{0}^{+}+\hat{G}_{0}^{+} \hat{W} \hat{G}_{0}^{+} \hat{W} \hat{G}_{0}^{+}+\cdots=\hat{G}^{+}(E)
$$

and

$$
\hat{G}^{+}(E)=(E+i \epsilon-\hat{H})^{-1}
$$

where $\hat{G}^{+}(E)$ the Green's function for the total Hamiltonian.

We can further use the identity in Eq. (2.6)

$$
\hat{W}|\phi\rangle=\left(\hat{W}+\hat{H}_{0}-E\right)|\phi\rangle=(\hat{H}-E)|\phi\rangle
$$

Using Eq. (2.8) and Eq. (2.11) putting this in Eq. (2.5) for the S-Matrix, we have

$$
S(E)=S_{0}(E)+\frac{i}{\hbar}\langle\phi|\hat{H}-E| \phi\rangle+\frac{i}{\hbar}\left\langle(\hat{H}-E) \phi\left|G^{+}(E)\right|(\hat{H}-E) \phi\right\rangle
$$

The most significant thing about this expression is the fact that the total Hamiltonian is the only thing that makes an appearance. This tells us that the actual 
choice of DW Hamiltonian and hence the residual interaction component is irrelevant. Further, we recognize that the solution to the DW Hamiltonian $\phi(r)$ can be chosen in to be in any convenient approximate form as long as it still obeys the asymptotic conditions.

The Kohn Variational functional for the S-matrix is defined as the first two terms of the Eq. (2.12) where $\phi(r)$ is the variational trial wavefunction.

$$
S_{K}[\phi]=S_{0}(E)+\frac{i}{\hbar}\langle\phi|\hat{H}-E| \phi\rangle
$$

The parameters in the variational trial function can be appropriately determined to ensure an extremum value for the functional $S_{K}[\phi]$. This expression Eq. (2.13) is, then, the variational solution for the S-Matrix. If $\phi(r)$ is the exact scattering solution such that

$$
(\hat{H}-E)|\phi\rangle=0
$$

and $S_{0}(E)=S_{\text {exact }}$ then clearly $S_{K}[\phi]$ becomes exact too.

In practice, the variational result is, of course, non-exact. The correction term is the third term on the right of Eq. (2.12)

$$
\Delta S=\frac{i}{\hbar}\left\langle(\hat{H}-E) \phi\left|G^{+}(E)\right|(\hat{H}-E) \phi\right\rangle
$$

At this stage, it is clear that evaluating this correction term and doing so exactly amounts to solving the Schrödinger equation for the system. The relevant questions 
to ask are if we can obtain an approximate result for this term and if such a result will prove useful.

We see that Eq. (2.15) contains the Green's function for the total Hamiltonian. The Green's function can be obtained from the time propagator as

$$
G^{+}(E)=(i \hbar)^{-1} \int_{0}^{\infty} d t e^{-\epsilon t} e^{i E t / \hbar} e^{-i \hat{H} t / \hbar}
$$

We note here that the Green's function $G^{+}(E)$ has a positive superscript because we have chosen to contour integrate around the poles of the function in the upper half of the complex plane. In other words, we have picked the form

$$
G^{+}(E)=\frac{1}{E-\hat{H}+i \epsilon}
$$

rather than

$$
G^{-}(E)=\frac{1}{E-\hat{H}-i \epsilon}
$$

Returning to Eq. (2.16), we see that SC-IVR can jump in here and do its part in evaluating the propagator. The overall result is therefore a semiclassical correction to a QM (variational) S-Matrix.

$$
S(E)=S_{K}[\phi]+\Delta S_{S C}
$$

The results of such a calculation have been shown to be good. ${ }^{47}$ This is ,therefore, our inspiration in trying to formulate a semiclassical energy correction to an approximate QM energy.

$$
E=E_{Q M}+\Delta E_{S C}
$$




\section{II.2 SC Correction for the Energy}

The eigenvalue equation for the energy is

$$
\hat{H}|\psi\rangle=E|\psi\rangle
$$

The exact solution to this equation can be obtained by diagonalizing the Hamiltonian which would then give us the eigenfunctions and their corresponding eigenenergies. A QM variational approach to this same problem, would start with a guess for the wavefunction $\phi$ and then minimize the energy $E[\phi]$ with respect to the different parameters of the trial wavefunction. We can easily prove that the variational energy thus calculated always approaches the exact result from above. ${ }^{48}$ We now wish to re-write this eigenvalue equation as a series expansion of sorts, where the first term(s) will correspond to the variational (or any other QM) approximation and the remaining terms form the 'correction'.

The total Hamiltonian is written as a sum of a perturbative term and a zeroth order Hamiltonian which has a known, exact solution

$$
\hat{H}=\hat{H}_{0}+\hat{W}
$$

Note that, the trial or 'guess' wavefunction (for the variational calculation) is usually taken to be an eigenstate of such a zeroth order Hamiltonian.

$$
\hat{H}_{0}|\phi\rangle=E_{0}|\phi\rangle
$$


A projection operator can now be defined for the unperturbed space as $\hat{P}=|\phi\rangle\langle\phi|$. The inverse space is then defined by $\hat{Q}=1-|\phi\rangle\langle\phi|$. Note that the sum of the projection operator and its inverse defines all space.

$$
\hat{P}+\hat{Q}=\mathbf{1}
$$

One other important property of the projection operators we will be using is

$$
\hat{P} \cdot \hat{P}=\hat{P}=\hat{P}^{+}
$$

and

$$
\hat{Q} \cdot \hat{Q}=\hat{Q}=\hat{Q}^{+}
$$

We now express the total wavefunction in terms of the projection operators defined above as

$$
|\psi\rangle=(\hat{P}+\hat{Q})|\psi\rangle=\psi_{P}+\psi_{Q}
$$

where we have defined $\hat{P}|\psi\rangle=\psi_{P}$ and $\hat{Q}|\psi\rangle=\psi_{Q}$

The eigenvalue equation can now be written as

$$
\hat{H}|\psi\rangle=\hat{H}(\hat{P}+\hat{Q})|\psi\rangle=E|\psi\rangle
$$

This equation can be projected into P-space and Q-space respectively, giving us two equations which can then be solved simultaneously. This is easily accomplished by operating on both sides of Eq. (2.26) with the corresponding projection operators. 
Using the property described in Eq. (2.23) and Eq. (2.24) and also the definitions from Eq. (2.25), we get

$$
\begin{array}{r}
(\hat{P} \hat{H} \hat{P}+\hat{P} \hat{H} \hat{Q})|\psi\rangle=E \hat{P}|\psi\rangle \\
\hat{H}_{P P} \psi_{P}+\hat{H}_{P Q} \psi_{Q}=E \psi_{P}
\end{array}
$$

Similarly,

$$
\begin{array}{r}
(\hat{Q} \hat{H} \hat{P}+\hat{Q} \hat{H} \hat{Q})|\psi\rangle=E \hat{Q}|\psi\rangle \\
\hat{H}_{Q P} \psi_{P}+\hat{H}_{Q Q} \psi_{Q}=E \psi_{Q}
\end{array}
$$

Rearranging Eq. (2.28),

$$
\psi_{Q}=\left(E-\hat{H}_{Q Q}\right)^{-1} \hat{H}_{Q P} \psi_{P}
$$

and substituting back into Eq. (2.27), we are left with

$$
\hat{H}_{P Q}\left(E-\hat{H}_{Q Q}\right)^{-1} \hat{H}_{Q P} \psi_{P}=\left(E-\hat{H}_{P P}\right) \psi_{P}
$$

Moving around the terms in Eq. (2.30), we obtain an expression for energy

$$
E \psi_{P}=\left(\hat{H}_{P P}+\hat{H}_{P Q}\left(E-\hat{H}_{Q Q}\right)^{-1} \hat{H}_{Q P}\right) \psi_{P}
$$

or, equivalently

$$
E=\langle\phi|\hat{H}| \phi\rangle+\left\langle\phi\left|\hat{H} \hat{Q}\left(E-\hat{H}_{Q Q}\right)^{-1} \hat{Q} \hat{H}\right| \phi\right\rangle
$$

The first term in Eq. (2.32) corresponds to a variational result, and the rest of the expression then becomes the correction term. 
An Unprojected Green's function The energy expression in Eq. (2.32) involves a Green's function $\left(E-\hat{H}_{Q Q}\right)^{-1}$ which is the total Green's function projected into the space of operator $\hat{Q}$. We would like an expression that is independent of the projection operators so that the we are not restricted to any specific choice of $\phi$ and hence $\hat{H}_{0}$ or $\hat{W}$

In trying to find the relationship between the projected and total Green's function, we use an operator expansion of the form

$$
(\hat{X}-\hat{Y})^{-1}=\hat{X}^{-1}+\sum_{n=0}^{\infty} \hat{X}^{-1}\left(\hat{Y} \hat{X}^{-1}\right)^{n}
$$

The expansion series for the projected Green's function

$$
\begin{aligned}
(E-\hat{Q} \hat{H} \hat{Q})= & {\left.\left[\left(E-\hat{Q} \hat{H}_{0} \hat{Q}\right)-\hat{Q} \hat{W} \hat{Q}\right)\right]^{-1} } \\
= & \left(E-\hat{Q} \hat{H}_{0} \hat{Q}\right)^{-1}+ \\
& \sum_{n=0}^{\infty}\left(E-\hat{Q} \hat{H}_{0} \hat{Q}\right)^{-1}(\hat{Q} \hat{W} \hat{Q})\left(E-\hat{Q} \hat{H}_{0} \hat{Q}\right)^{-1}
\end{aligned}
$$

Alternatively, we can use the Brillouin-Wigner perturbation theory ${ }^{49,50}$ to expand the projected Green's function in a similar fashion. We now have,

$$
\frac{1}{E-\hat{H}_{Q Q}}=\frac{\hat{Q}}{E-\hat{H}_{0}}+\frac{\hat{Q}}{E-\hat{H}_{0}} \hat{W} \frac{\hat{Q}}{E-\hat{H}_{0}}+\ldots
$$


The correction term is, therefore,

$$
\begin{aligned}
\Delta E= & \langle\phi|(\hat{H}-<\hat{H}>) \hat{Q} \\
& \left(\frac{\hat{Q}}{E-\hat{H}_{0}}+\frac{\hat{Q}}{E-\hat{H}_{0}} \hat{W} \frac{\hat{Q}}{E-\hat{H}_{0}}+\ldots\right) \\
& \hat{Q}(\hat{H}-<\hat{H}>)|\phi\rangle
\end{aligned}
$$

where $<\hat{H}>=\langle\phi|\hat{H}| \phi\rangle$, the variational result; we have also used the equality

$$
\hat{Q} \hat{H}|\phi\rangle=\hat{Q}(\hat{H}-<\hat{H}>)|\phi\rangle
$$

and we know that $\hat{Q}|\phi\rangle=0$ since we chose an orthogonal basis. The series to be summed to infinite order to find $\Delta E$

$$
\Delta E=\Delta E_{1}+\Delta E_{2}+\Delta E_{3}+\ldots
$$

By examining the first few terms individually we can identify a pattern that can then be used to find the infinite order sum.

$$
\Delta E_{1}=\left\langle\phi\left|(\hat{H}-<\hat{H}>) \frac{\hat{Q}}{E-\hat{H}_{0}}(\hat{H}-<\hat{H}>)\right| \phi\right\rangle
$$

We explicitly write the unprojected Green's function as

$$
\frac{\hat{Q}}{E-\hat{H}_{0}}=\left(E-\hat{H}_{0}\right)^{-1}-\frac{|\phi\rangle\langle\phi|}{E-E_{0}}
$$

and substitute back in the expression for the first correction term in the series and note that

$$
\langle\phi|(\hat{H}-<\hat{H}>)| \phi\rangle=0
$$


This gives us

$$
\Delta E_{1}=\left\langle\phi\left|(\hat{H}-<\hat{H}>) \frac{1}{E-\hat{H}_{0}}(\hat{H}-<\hat{H}>)\right| \phi\right\rangle
$$

We find expressions for the second and third term in the series in a similar fashion

$$
\begin{aligned}
& \Delta E_{2}=\left\langle\phi\left|(\hat{H}-<\hat{H}>) \hat{G}_{0} \hat{W} \hat{G}_{0}(\hat{H}-<\hat{H}>)\right| \phi\right\rangle \\
& \Delta E_{3}=\left\langle\phi\left|(\hat{H}-<\hat{H}>) \hat{G}_{0} \hat{W} \hat{G}_{0} \hat{W} \hat{G}_{0}(\hat{H}-<\hat{H}>)\right| \phi\right\rangle-\frac{\Delta E_{1}^{2}}{E-E_{0}}
\end{aligned}
$$

The sum of these three terms is then

$$
\begin{aligned}
\Delta E_{1}+\Delta E_{2}+\Delta E_{3}= & \langle\phi|\left(\hat{H}-<\hat{H}>-\Delta E_{1}\right) \\
& \left(\hat{G}_{0}+\hat{G}_{0} \hat{W} \hat{G}_{0}\right)\left(\hat{H}-<\hat{H}>-\Delta E_{1}\right)|\phi\rangle
\end{aligned}
$$

We now begin to see what the higher order terms will be like.

$$
\begin{aligned}
\Delta E=\quad & \langle\phi|\left(\hat{H}-<\hat{H}>-\left(\Delta E_{1}+\Delta E_{2}+\ldots\right)\right. \\
& \left(\hat{G}_{0}+\hat{G}_{0} \hat{W} \hat{G}_{0}+\hat{G}_{0} \hat{W} \hat{G}_{0} \hat{W} \hat{G}_{0}+\ldots\right) \\
& \left(\hat{H}-<\hat{H}>-\left(\Delta E_{1}+\Delta E_{2}+\ldots\right)\right)|\phi\rangle
\end{aligned}
$$

The series involving the zeroth order Green's functions and the 'perturbation' term $\hat{W}$ is the Born series expansion for the unprojected Green's function of the total Hamiltonian from Eq. (2.9) The infinite sum for $\Delta E$, using this formal sum of the DW Born series gives us the final expression for the correction term.

$$
\Delta E(E)=\langle\phi|(\hat{H}-<\hat{H}>-\Delta E) G(E)(\hat{H}-<\hat{H}>-\Delta E)| \phi\rangle
$$


This expression is now independent of the way we partition the Hamiltonian. We can, therefore, choose $\hat{H}_{0}$ to be any convenient part of $\hat{H}$ that we then solve by, say, variational methods. The variational energy is represented by $\langle\hat{H}>$ in Eq. (2.48) The projection operators are then defined in terms of the subspace of the variational solutions and the space orthogonal to that.

After all that work, let make on simple check to verify that our expression for the correction term is exact. The total energy is

$$
E=<\hat{H}>+\Delta E
$$

Substituting for $\Delta E$ with Eq. (2.48) and using Eq. (2.49) within that, we have

$$
\begin{aligned}
E & =\left\langle\hat{H}>+\left\langle\phi\left|(\hat{H}-E)(E-\hat{H})^{-1}(\hat{H}-E)\right| \phi\right\rangle\right. \\
& =\langle\hat{H}>-\langle\phi|\hat{H}-E| \phi\rangle \\
& =<\hat{H}>-<\hat{H}>+E \\
& =E
\end{aligned}
$$

This equation is an Identity.

We can evaluate the Green's function semiclassically as described before. The final step in this derivation is to obtain $\Delta E$ as a solution to a quadratic equation.

$$
\Delta E=G_{22}(E)+\Delta E^{2} G_{11}(E)-2 \Delta E G_{12}(E)
$$


where, we define

$$
\begin{aligned}
& G_{11}(E)=\langle\phi|G(E)| \phi\rangle \\
& G_{22}(E)=\langle\phi|(\hat{H}-<\hat{H}>) G(E)(\hat{H}-<\hat{H}>)| \phi\rangle \\
& G_{12}(E)=\langle\phi|G(E)(\hat{H}-<\hat{H}>)| \phi\rangle
\end{aligned}
$$

We can solve this quadratic equation to explicitly obtain $\Delta E(E)$

$$
\Delta E(E)=\frac{G_{12}+\frac{1}{2} \pm \sqrt{\left(G_{12}+\frac{1}{2}\right)^{2}-G_{11} G_{22}}}{G_{11}}
$$

The solution is thus obtained graphically by plotting the left and right sides of Eq. (2.53) or its equivalent equation for the total energy obtained by adding $<\hat{H}>$ to both sides - and finding the point of intersection. The equation which we will be plotting is thus

$$
\begin{aligned}
E & =<\hat{H}>+\frac{G_{12}+\frac{1}{2} \pm \sqrt{\left(G_{12}+\frac{1}{2}\right)^{2}-G_{11} G_{22}}}{G_{11}} \\
& =F(E)
\end{aligned}
$$

\section{Application}

In practice we define the correction term a little differently in order to make the expression a little less unwieldy. We start with Eq. (2.50) and obtain

$$
\begin{aligned}
\Delta E & =\langle\phi|(\hat{H}-E) G(E)(\hat{H}-E)| \phi\rangle \\
\Delta E & =E^{2} G_{11}(E)-2 E G_{12}(E)+G_{22}(E)
\end{aligned}
$$


The modified Green's functions are now defined as

$$
\begin{aligned}
G_{11}(E) & =\langle\phi|G(E)| \phi\rangle \\
G_{12}(E) & =\langle\phi|\hat{H} G(E)| \phi\rangle \\
G_{22}(E) & =\langle\phi|\hat{H} G(E) \hat{H}| \phi\rangle
\end{aligned}
$$

The HK-IVR expression for the propagator (see Chapter 1 for details) is

$$
e^{-i \hat{H} t / \hbar}=\int d p_{0} \int d q_{0}\left|p_{t}, q_{t}\right\rangle e^{\frac{i}{\hbar} S_{t}\left(q_{0}, p_{0}\right)} C_{t}\left(p_{0}, q_{0}\right)\left\langle p_{0}, q_{0}\right|
$$

Using this in the expression for $G_{11}(E)$ we have

$$
G_{11}(E)=\frac{1}{i \hbar} \int_{0}^{\infty} e^{i(E+i \epsilon) t / \hbar}\left\langle\phi \mid p_{t}, q_{t}\right\rangle e^{\frac{i}{\hbar} S_{t}\left(q_{0}, p_{0}\right)} C_{t}\left(p_{0}, q_{0}\right)\left\langle p_{0}, q_{0} \mid \phi\right\rangle
$$

Similarly for the other terms, we have

$$
\begin{aligned}
G_{12}(E) & =\frac{1}{i \hbar} \int_{0}^{\infty} e^{i(E+i \epsilon) t / \hbar}\left\langle\phi|\hat{H}| p_{t}, q_{t}\right\rangle e^{\frac{i}{\hbar} S_{t}\left(q_{0}, p_{0}\right)} C_{t}\left(p_{0}, q_{0}\right)\left\langle p_{0}, q_{0} \mid \phi\right\rangle \\
G 22(E) & =\frac{1}{i \hbar} \int_{0}^{\infty} e^{i(E+i \epsilon) t / \hbar}\left\langle\phi|\hat{H}| p_{t}, q_{t}\right\rangle e^{\frac{i}{\hbar} S_{t}\left(q_{0}, p_{0}\right)} C_{t}\left(p_{0}, q_{0}\right)\left\langle p_{0}, q_{0}|\hat{H}| \phi\right\rangle(3.60)
\end{aligned}
$$

The terms in Eq. (3.60) that do not occur in the expression for $G_{11}(E)$ can be evaluated analytically. If we assume the most general form of $\phi$ to be a coherent state $\left|p_{i}, q_{i}\right\rangle$, we can define two functions $f_{1}$ and $f_{2}$ such that

$$
\begin{aligned}
\langle\phi|\hat{H}| p, q\rangle & =f_{1}\left(p, q, p_{i}, q_{i}\right)\langle\langle\phi|| \mid p, q\rangle\rangle \\
\langle p, q|\hat{H}| \phi\rangle & =f_{2}\left(p, q, p_{i}, q_{i}\right)\langle\langle p, q \mid \| \phi\rangle\rangle
\end{aligned}
$$

For the specific examples in this section, we analytically obtain the form of $f_{1}$ and $f_{2}$ and show that these involve no extra effort over and above the calculation of $G_{11}(E)$. 


\section{III.1 The Anharmonic Oscillator Model}

The anharmonic oscillator - a harmonic oscillator with a quartic anharmonicity has served as a good testing ground for energy calculation methods. ${ }^{51}$

The potential we use is the form

$$
V=\frac{1}{2} m \omega^{2} x^{2}+\lambda x^{4}
$$

The QM variational result is again obtained analytically here - we evaluate the expectation value of the Hamiltonian with a ground state wavefunction (a coherent state, in this case) and then minimize the energy with respect to the width of the coherent state. More generally, for a real system, the expectation value will be calculated by a variational approach such as the SCF method.

$$
\left\langle p_{i}, q_{i}|\hat{H}| p_{i}, q_{i}\right\rangle=\frac{\gamma}{4}+\frac{1}{4 \gamma}+\frac{3 \lambda}{4 \gamma^{2}}
$$

where

$$
\left\langle x \mid p_{i}, q_{i}\right\rangle=\left(\frac{\gamma}{\pi}\right)^{1 / 4} e^{-\frac{\gamma}{2}\left(x-q_{i}\right)^{2}} e^{\frac{i}{\hbar} p_{i}\left(x-q_{i}\right)}
$$

We choose our reference state to be a simple gaussian i.e, a coherent state where

$$
\begin{aligned}
& p_{i}=0 \\
& q_{i}=0
\end{aligned}
$$

We now minimise Eq. (3.64) with respect to $\gamma$

$$
\gamma^{3}-\gamma-6 \lambda=0
$$


The $\gamma$ value therefore depends on the degree of anharmonicity as described by $\lambda$ For the results shown here $\lambda=1.0$ and $\gamma=2 . d 0$.

We evaluate the correction term for this potential using Eq. (2.53). The terms $f_{1}$ and $f_{2}$ from Eq. (3.61) and Eq. (3.62) are calculated and broken down in terms of the contributions from each of the parts of the Hamiltonian. These equations are for the most general choice of both the reference state $\phi$ and the HK-IVR coherent states $\left\langle p_{1}, q_{1}|\hat{H}| p_{2}, q_{2}\right\rangle$ The contribution from the kinetic energy term is

$$
K E=-\frac{1}{2} \frac{\hbar^{2}}{2 m}\left(-\frac{\gamma}{2}+\gamma^{2} A^{2}\right)
$$

where,

$$
A=\frac{1}{2}\left(q_{1}+q_{2}\right)-\frac{i}{2 \hbar \gamma}\left(p_{1}-p_{2}\right)
$$

The harmonic contribution is

$$
H O=\frac{1}{2} m \omega^{2}\left(\frac{1}{2} \gamma+A^{2}\right)
$$

and finally, the quartic contribution is

$$
Q O=\lambda\left(\frac{3}{4} \gamma^{2}+\frac{3}{\gamma} A^{2}+A^{4}\right)
$$

The function $f_{1 / 2}=K E+H O+Q O$

The choice of subscript 1 or 2 is decided by our choice of $p_{1}, q_{1}$ and $p_{2}, q_{2}$. $f_{1}$ is obtained by setting $p_{1}=p_{i}=0, q_{1}=q_{i}=0$ and $p_{2}=p_{t}, q_{2}=q_{t}$. 
$f_{2}$ is obtained by setting $p_{1}=p_{0}, q_{1}=q_{0}$ and $p_{2}=p_{i}=0, q_{2}=q_{i}=0$.

\section{III.2 The Quartic Oscillator}

We now look at a different and somewhat harder (more anharmonic) case to see if these problem are recurring. One reason for doing this, is that in the previous model, the energy correction term itself is very small, making numerical errors that much more likely to interfere with the result. The potential for this model problem is

$$
V(x)=\lambda x^{4}
$$

The variational result for this case is

$$
\left\langle p_{i}, q_{i}|\hat{H}| p_{i}, q_{i}\right\rangle=\frac{\gamma}{4}+\frac{3 \lambda}{4 \gamma^{2}}
$$

Minimizing this energy, we have an equation relating $\gamma$ to the $\lambda$ value.

$$
\frac{1}{4}-\frac{6 \lambda}{4 \gamma^{3}}=0
$$

We choose one $\lambda$ values to study this model $\lambda=0.7$ and $\gamma=1.6134$

The $f_{1}$ and $f_{2}$ functions that are part of the modified Green's functions are the same as defined previously in Eq. (3.61) and Eq. (3.62), except that we no longer 
have the harmonic contribution to $f_{1 / 2}$.

$$
f_{1 / 2}=K E+Q O
$$

where we get $K E$ and $Q O$ from Eq. (3.68) and Eq. (3.71).

\section{III.3 The Harmonium Atom - A Two Electron Model}

Our last model is a multidimensional one. The harmonium atom is used by theorists as a model of a two electron atom with harmonic potentials and a coupling term between them.

$$
\hat{H}=\frac{-\hbar^{2}}{2 m}\left(\nabla_{r_{1}}^{2}+\nabla_{r_{2}}^{2}\right)+\frac{1}{2} k\left(r_{1}^{2}+r_{2}^{2}\right)+\frac{1}{r_{12}}
$$

In sum and difference coordinates $R=\frac{1}{2}\left(r_{1}+r_{2}\right)$ and $r=r_{1}-r_{2}$

We can re-write the total Hamiltonian as

$$
\hat{H}=\hat{H}_{\mathbf{R}}+\hat{H}_{\mathbf{r}}
$$

where we define

$$
\hat{H}_{\mathbf{R}}=\frac{-\hbar^{2}}{4 m} \nabla_{\mathbf{R}}^{2}+k \mathbf{R}^{2}
$$

and

$$
\hat{H}_{\mathbf{r}}=\frac{-\hbar^{2}}{4 m} \nabla_{\mathbf{r}}^{2}+k \mathbf{r}^{2}+\frac{\mathbf{1}}{\mathbf{r}}
$$

Note that we are working in atomic units and for the electron $\hbar=1$ and $m=1$ 
The Hamiltonian approximates an atom with nuclear charge $Z$ when

$$
\begin{aligned}
k & =\omega^{2} \\
\omega & =\frac{16 Z^{2}}{9 \pi}
\end{aligned}
$$

The exact ground state energies have been calculated for the $Z=1$ case (a $H^{-}$ atom) and for the $Z=2$ case (a He atom). ${ }^{52,53}$

The 'trial' wavefunction we use for the variational process is just a gaussian in the coordinates for both electrons. We also make the change of variables to the $\mathbf{r}$ and $\mathbf{R}$ coordinates.

$$
\begin{aligned}
\phi_{0}\left(\mathbf{r}_{1}, \mathbf{r}_{2}\right) & =\frac{\alpha^{3 / 2}}{\pi} e^{-\alpha\left(\mathbf{r}_{1}^{2}+\mathbf{r}_{2}^{2}\right)} \\
& =\chi(\mathbf{R}) \psi(\mathbf{r}) \\
\chi(\mathbf{R}) & =\left(\frac{2 \alpha}{\pi}\right)^{3 / 4} e^{-\alpha \mathbf{R}^{2}} \\
\psi(\mathbf{r}) & =\left(\frac{\alpha}{\pi}\right)^{3 / 4} e^{-\frac{\alpha}{4} \mathbf{r}^{2}}
\end{aligned}
$$

The variational results for the harmonium atom are obtained from

$$
\begin{aligned}
\left\langle\chi(\mathbf{R})\left|\hat{\mathbf{H}}_{\mathbf{R}}\right| \chi(\mathbf{R})\right\rangle & =\frac{3}{4}\left(\frac{\hbar^{2} \alpha}{m}+\frac{k}{\alpha}\right) \\
\left\langle\psi(\mathbf{r})\left|\hat{\mathbf{H}}_{\mathbf{r}}\right| \psi(\mathbf{r})\right\rangle & =\frac{3}{4}\left(\frac{\hbar^{2} \alpha}{m}+\frac{k}{\alpha}\right)+\left(\frac{2 \alpha}{\pi}\right)^{\frac{1}{2}}
\end{aligned}
$$

by minimizing these individual functions with respect to the appropriate $\alpha$ and $k$ values. 
Once again, we are able to evaluate the modified Green's functions $G_{12}$ and $G_{22}$ analytically.

$$
\begin{aligned}
\left\langle p, q ; P, Q|\hat{H}| \phi_{0}\right\rangle & =\left\langle P, Q\left|\hat{H}_{R}\right| \chi\right\rangle\langle p, q \mid \psi\rangle+\langle P, Q \mid \chi\rangle\left\langle p, q\left|\hat{H}_{r}\right| \psi\right\rangle \\
& =F(p, q ; P, Q)\langle p, q \mid \psi\rangle\langle P, Q \mid \chi\rangle
\end{aligned}
$$

where $F(p, q ; P, Q)$ is defined as

$$
\begin{aligned}
F(p, q ; P, Q) & =\frac{3}{2 m} \cdot \frac{\alpha \gamma_{1}}{2 \alpha+\gamma_{1}}+\frac{1}{\left(2 \alpha+\gamma_{1}\right)^{2}}\left(k-\frac{\alpha^{2}}{m}\right)\left|\gamma_{1} Q-i P\right|^{2} \\
& +\frac{3}{m} \frac{\alpha \gamma_{2}}{\alpha+2 \gamma_{2}}+\frac{3}{2} \frac{k}{\alpha+2 \gamma_{2}}+\frac{1}{\left(\alpha+2 \gamma_{2}\right)^{2}}\left(k-\frac{\alpha^{2}}{m}\right)\left|\gamma_{2} q-i p\right|^{2} \\
& +\frac{\alpha / 2+\gamma_{2}}{\left|\gamma_{2} q-i p\right|} \operatorname{erf}\left[\frac{\left|\gamma_{2} q-i p\right|}{\left(\alpha / 2+\gamma_{2}\right)^{\frac{1}{2}}}\right]
\end{aligned}
$$

The error function erf is defined here as

$$
\begin{aligned}
I & =\int d^{3} r \frac{1}{r} e^{-\alpha|r-b|^{2}} \\
& =\left(\frac{\pi}{\alpha}\right)^{3 / 2} \frac{1}{|b|} \operatorname{erf}(\sqrt{\alpha}|b|)
\end{aligned}
$$

This expression is rather tedious looking, but computationally simple enough to incorporate. We note that the 1st part of the expression Eq. (3.83) is entirely devoted to the center of mass variable $\mathbf{R}$; the rest of the expression is entirely a function of the difference variable $\mathbf{r}$, Eq. (3.84). This allows us to evaluate the two factors independently, simplifying the calculation quite a lot. 


\section{Discussion}

\section{IV.1 Anharmonic Oscillator}

The Anharmonic oscillator with $\lambda=1.0$ is the first case we look at. The results are shown in Figure 3.1, and since we can rescale the Hamiltonian in terms of $\lambda$, one case is considered sufficiently representative of the system. The figures shown here also have parameters for the harmonic component $m=1$ and $\omega=1$. The reference state is a coherent state of width $\gamma=2.0$, chosen as described in Eq. (3.67) The corrected energy is obtained from the graphical intersection of the two sides of our Identity equation Eq. (2.54). The exact energy for this system is calculated by the

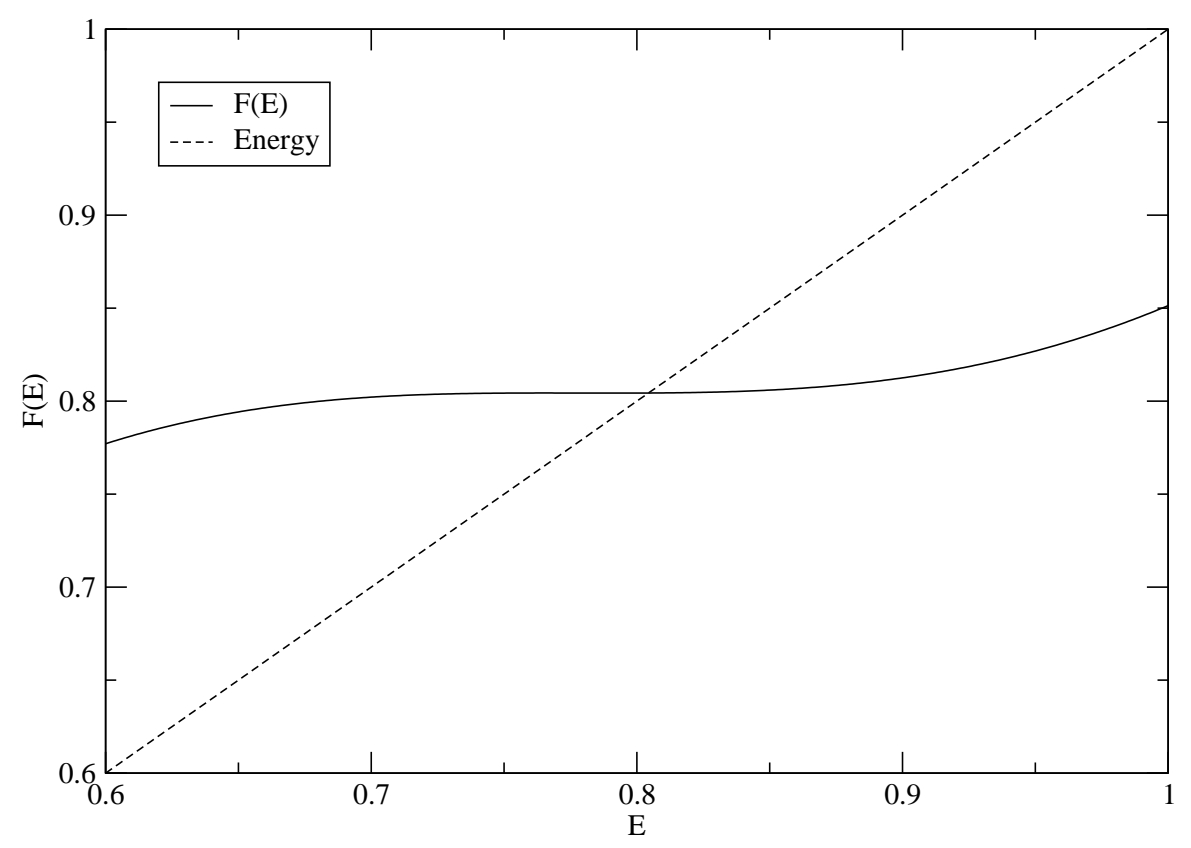

Figure 3.1: The intersection of $\mathrm{F}(\mathrm{E})$ and $\mathrm{E}$ for the $\epsilon=0.01$ case 
Discrete Variable Representation (DVR) method described in. ${ }^{54}$

Table 3.1: Results for the anharmonic oscillator with $\gamma=2$ and $\epsilon=0.01$

\begin{tabular}{|c|c|c|}
\hline Exact Energy & $E_{D V R}$ & 0.8038 \\
\hline Variational Energy & $<\hat{H}>$ & 0.8125 \\
\hline Corrected Energy & $E_{S C}$ & 0.8043 \\
\hline
\end{tabular}

The percentage of the exact correction $\Delta E$ that our method captures is thus $88.5 \%$ which is in good agreement with our original guess. The percentage error for the Corrected Energy is $0.12 \%$ as opposed to the percentage error for just the Variational Energy which is around $1.1 \%$ from the values in Table 3.1 Although in this case, it is clear that we would be quite content with the variational result, we show that the corrected energy is a factor of 10 more accurate and this would be a huge improvement for problems that variational methods find harder to deal with.

We would like, now, to take a closer look at the behavior of these functions over a broader range of energy. (In the previous case we only used a very small window ranging only \pm 0.5 around the variational energy)) Figure 3.2 shows us an effectively zoomed out version of Figure 3.1. In Figure 3.2, we see that there appear to be multiple points of intersection of the two curves, something that was not obvious when we looked at a narrower window of the Fourier transform. We know that the 


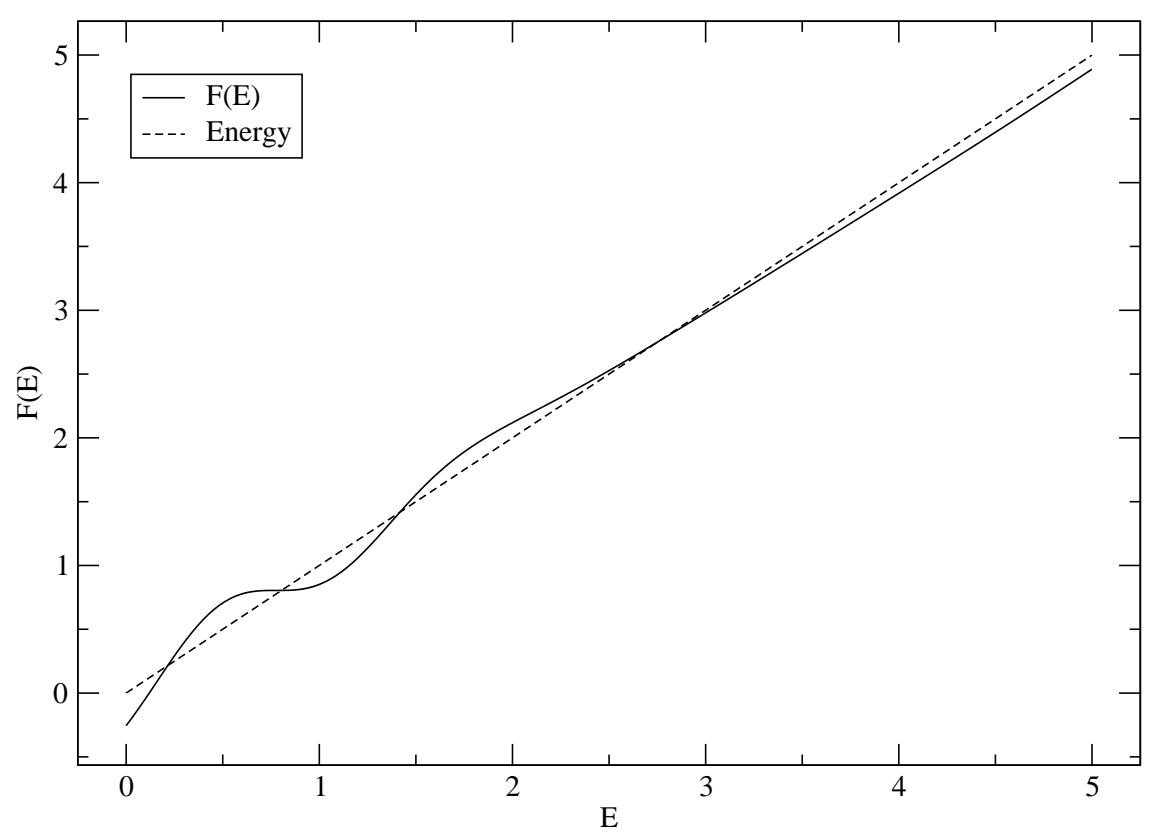

Figure 3.2: The intersection of $\mathrm{F}(\mathrm{E})$ and $\mathrm{E}$ for the $\epsilon=0.01$ case - Figure 3.1 over a larger energy window

variational result overestimates the exact value, so we are looking for a negative correction term This allows us to disregard all positive roots and only pick out the negative roots of Eq. (2.53). In other words, we only look at the values for the corrected energy that are below the variational energy. But, since our equation is pseudo-quadratic (where the coefficients are themselves functions of the variable) we are not entirely surprised to find more than one value, even after eliminating all options greater than the variational result. This raises the question of how to choose the 'right' intersection and how to identify a pattern that we can exploit to make the right choice consistently.

During the derivation we emphasized that our result, while derived as a correction 
to the variational result, does not make any demands on the accuracy of the primary calculation. It is, naturally, to our advantage to choose as accurate a starting point as possible, but as we show in Figure 3.3, we can improve upon any result by about $10 \%$.

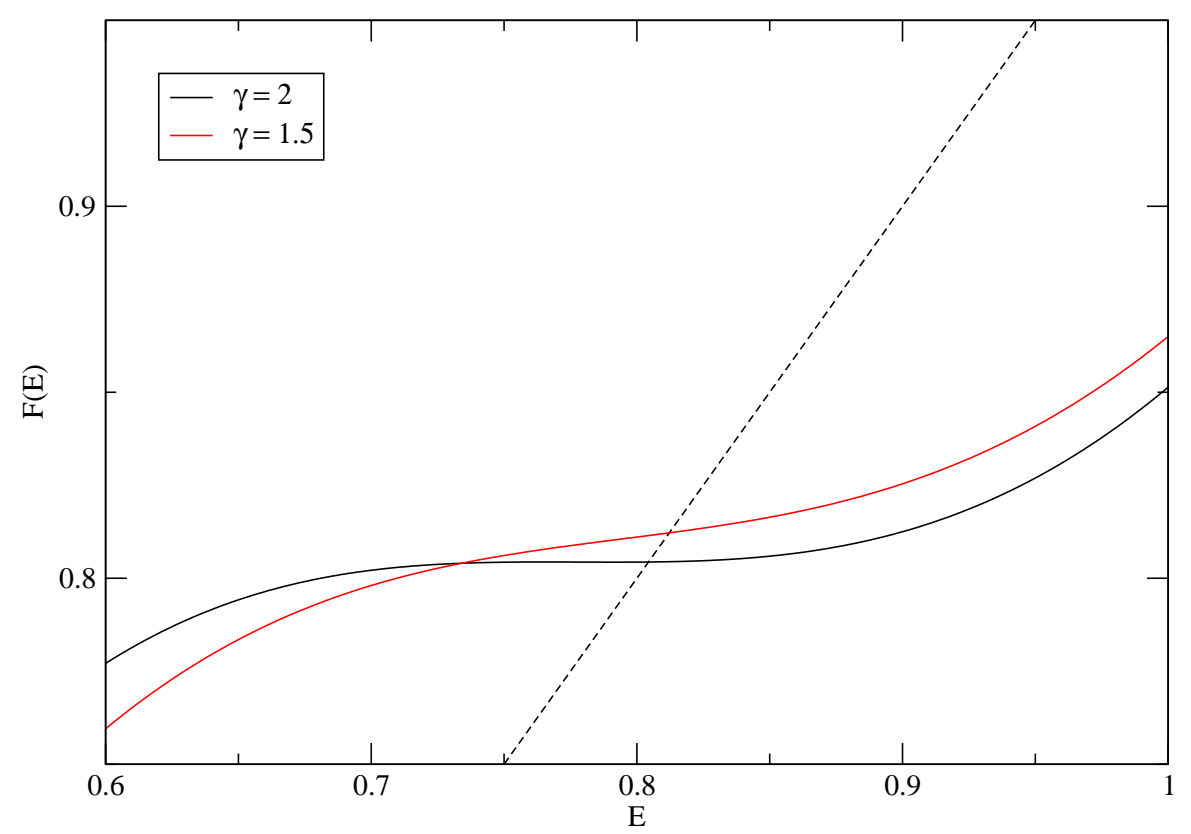

Figure 3.3: The same as Figure 3.1 but with a bad guess for the 'variational' wavefunction with $\gamma=1.5$ and $\epsilon=0.01$

In Figure 3.3 we choose to start with a coherent state of width $\gamma=1.5$ which is clearly not the best variational wavefunction. For this case, the various energy values obtained are shown in 3.2 (the Exact one is, of course, the same).

Percentage Error here for the variational result is $8.9 \%$ and for the SC-Corrected Energy it is about $1 \%$. We manage to recover almost $90 \%$ of the correction term in this case. 
There is one other parameter that we need to discuss. The Green's functions are calculated from the propagator by

$$
\hat{G}(E)=\frac{1}{i \hbar} \int_{0}^{\infty} d t e^{i(E+i \epsilon) t / \hbar} e^{-i \hat{H} t / h b a r}
$$

There is the factor $\epsilon$ that we use in the Fourier transform to dampen the oscillations (or another way of looking at this factor is that it is used to deal with the poles of the Green's function); ideally, $\epsilon \rightarrow 0$. In practice, we can choose $\epsilon$ to take on any value that allows us to smooth out our function and see features better. As

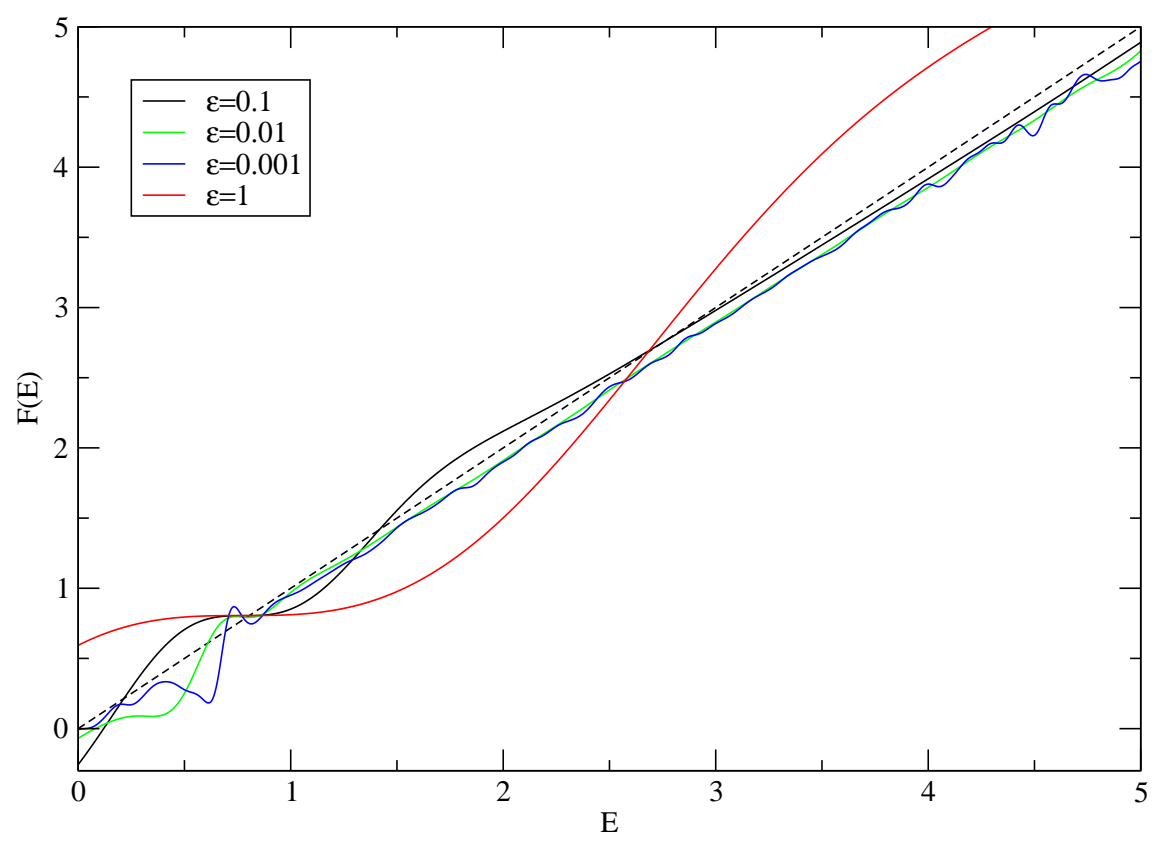

Figure 3.4: Anharmonic oscillator $\gamma=2$ and over a range of $\epsilon$ values. We look to see how much the accuracy of the calculation is affected by changing these values.

shown in Figure 3.4 we see that by influencing the smoothness of the function over a given interval, we automatically affect how many times it intersects the energy 
line. In this case, for instance over a range of energies from 0 to the variational result, only a large (relatively!) value of $\epsilon=1.0$ chooses out just one intersection point. We also note that in Figure 3.4, there is a common point at which all the curves cross the energy line, but a closer inspection shows Figure 3.5 that what appears to be the same point is actually a range of value from $0.78-0.802$ which is a sizeable error bar given the magnitude of numbers we are comparing. The most

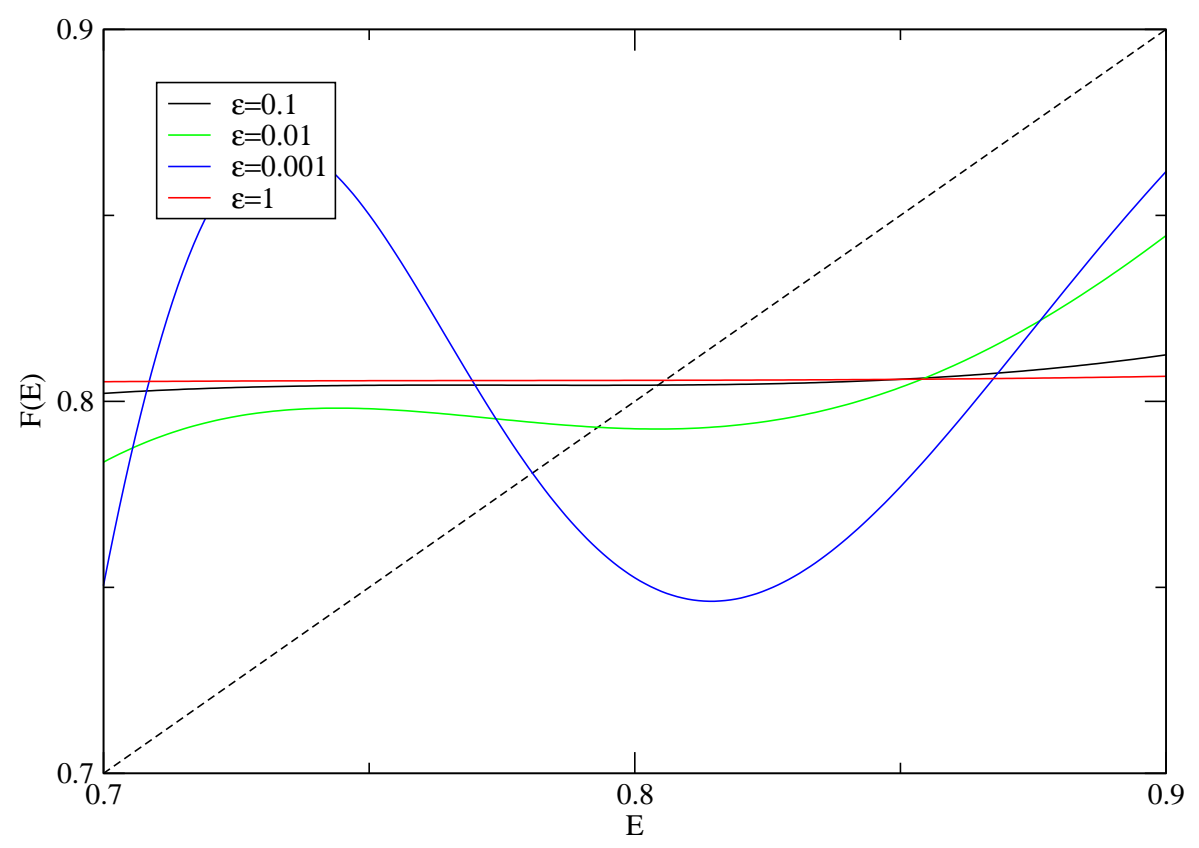

Figure 3.5: Zoomed in version of Figure 3.4

worrying here, is that given that we know the exact result, we would assume that the smallest value of $\epsilon$ that is in best keeping with our derivation where we look for the zero limit of this quantity will give the best result. We see that in this case, at least, the best value is from $\epsilon=0.01$ and not $\epsilon=0.001$. It also becomes clear 
that since our method is not bound from below by the exact energy. This may be a problem that is limited to the current case and simply a result of numerical error in the Fourier transform, but something we need to keep a close watch on as we analyze the results of the other models.

\section{IV.2 Quartic Oscillator}

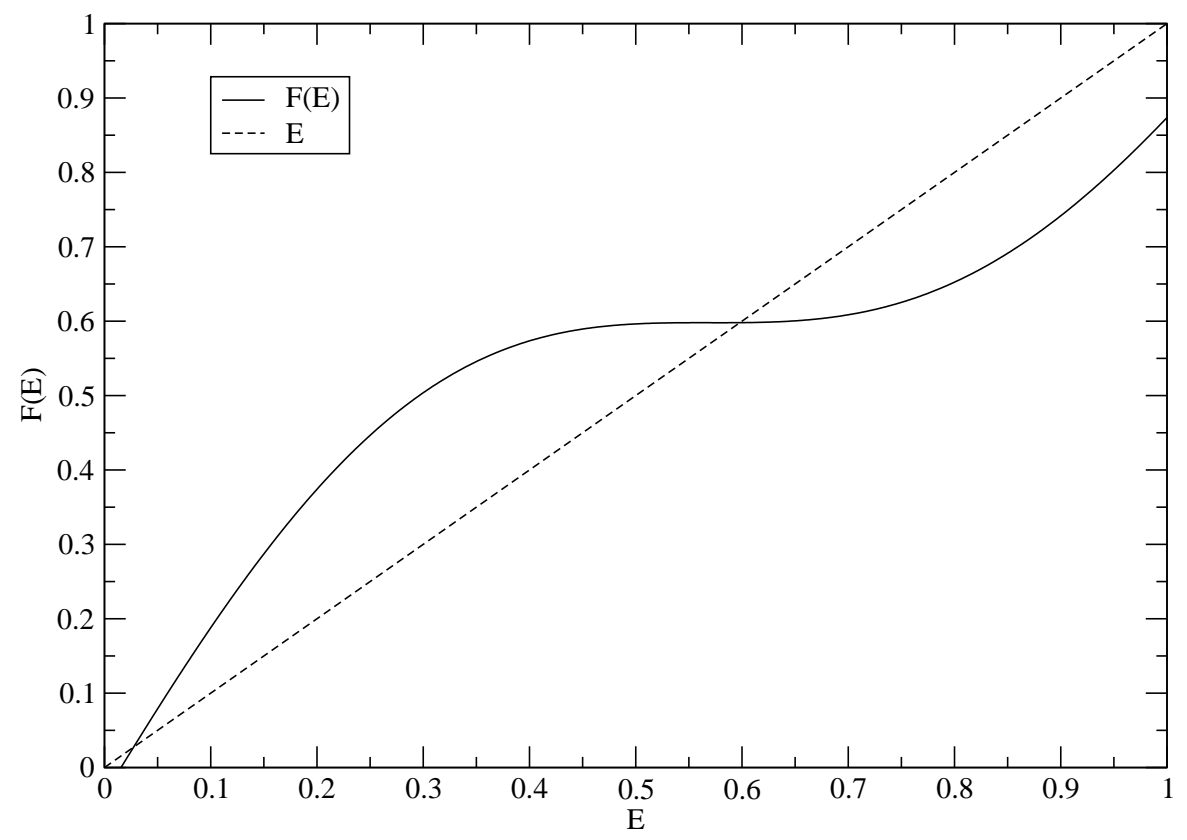

Figure 3.6: Energy of a Quartic Oscillator with $\gamma=1.61$ and $\epsilon=0.001$.

The Quartic Oscillator model is more challenging problem and we show the results of $\lambda=0.7$ case in Figure 3.6. The choice of this particular values is somewhat arbitrary. Again, since we can rescale our coordinates, one example is sufficient to study the model. For $\lambda=0.7$ 
The error percentages for the variational result and the SC-corrected energy are $2 \%$ and $0.02 \%$ respectively as shown in 3.3 . However, the choice of corrected energy once again involved using multiple epsilon values (shown in Figure 3.7 and 3.8) and a manual inspection of the graphs to decide which of the many intersection points was the relevant root. The one improvement over the previous anharmonic oscillator is that here, the smallest $\epsilon$ value that is still a relatively smooth function gives us the best result.

On the one hand, we are beginning to see what might pass for a pattern. The corrected energy (and therefore more accurate energy) appears to be simply the point where the two curves intersect that is the closest point lower than the variational energy. However, this is a somewhat ad-hoc solution though and it is hard to generalize this in a sensible manner to all systems, without stopping to check!

\section{IV.3 Harmonium Atom}

The last model system we look at is the Harmonium Atom. Figure 3.9 shows the results for the $k=0.25$ case; Figure 3.11 for $k=0.32$ and Figure 3.13 for $k=5.1$.

The Figures 3.10, 3.12, 3.14 zoom in on the point of intersection for the different $\epsilon$ values. The results are tabulated in 3.4. The last column in this table $\Delta E_{r} \%$ is the percentage of the correction term recovered semiclassically.

The various tables and figures all show that our method is quite effective in obtaining at least a part of the correlation energy for a variational calculation. 
Table 3.2: Results for anharmonic oscillator with $\gamma=1.5$ and $\epsilon=0.01$

\begin{tabular}{|c|c|c|}
\hline Variational Energy & $<\hat{H}>$ & 0.875 \\
\hline Corrected Energy & $E_{S C}$ & 0.812 \\
\hline
\end{tabular}

Table 3.3: Results for the Quartic Oscillator with $\gamma=1.61$ and $\epsilon=0.001$

\begin{tabular}{|c|c|c|}
\hline Exact Energy & $E_{D V R}$ & 0.5931 \\
\hline Variational Energy & $<\hat{H}>$ & 0.6050 \\
\hline Corrected Energy & $E_{S C}$ & 0.5930 \\
\hline
\end{tabular}

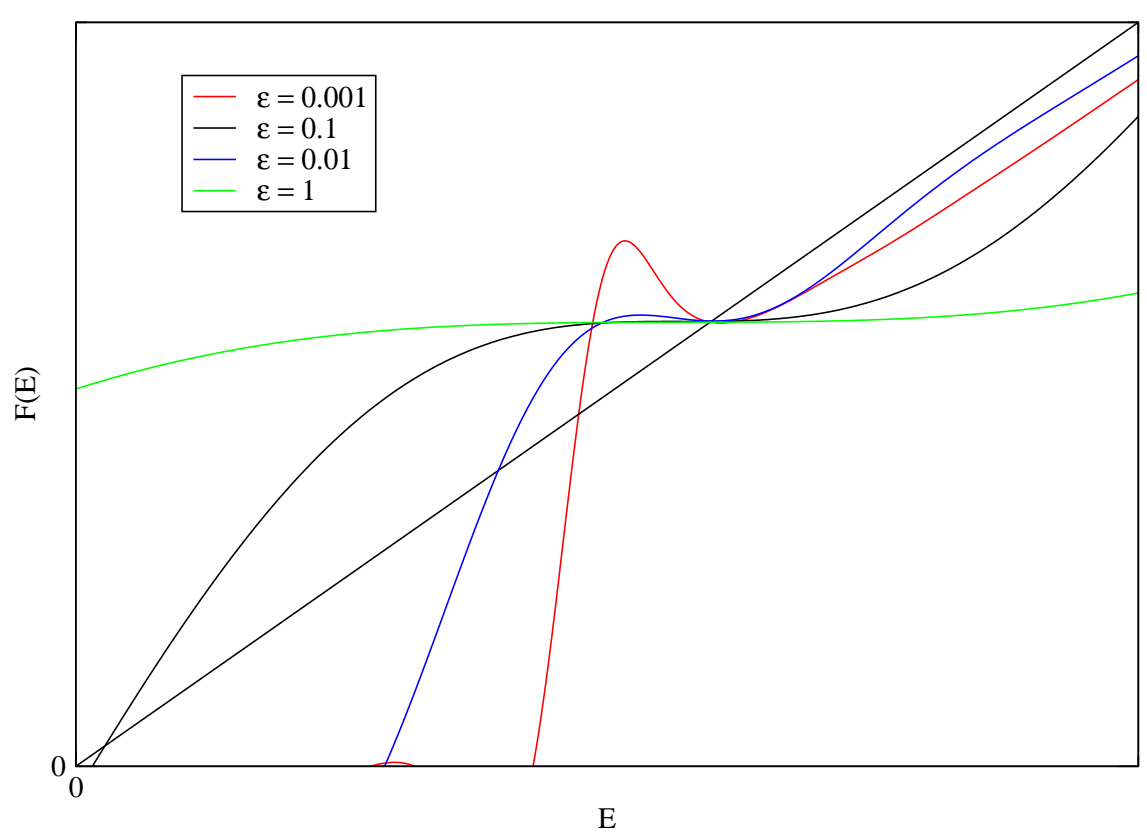

Figure 3.7: Quartic oscillator with $\gamma=1.61$ and $\epsilon=0.001$ with several $\epsilon$ values. 


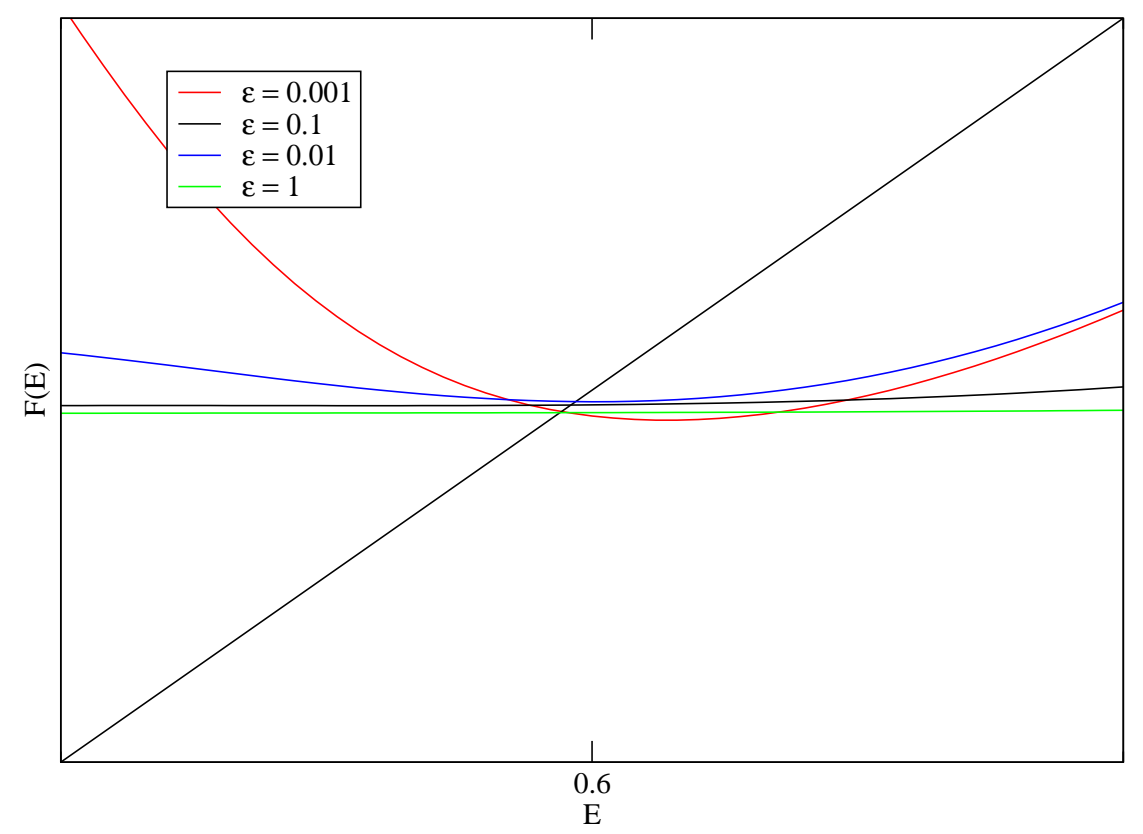

Figure 3.8: Figure 3.7 Zoomed in to see intersection point.

Table 3.4: Results for the Harmonium Atom with different $k$ values

\begin{tabular}{|c|c|c|c|c|c|c|c|}
\hline$k$ & $\gamma$ & $E_{D V R}$ & $<\hat{H}>$ & $E_{S C}$ & $\%$ Error $<\hat{H}>$ & $\%$ Error $E_{S C}$ & $\Delta E_{r} \%$ \\
\hline 0.25 & 0.42 & 2.00 & 2.04 & 2.01 & 2.00 & 0.50 & 75 \\
\hline 0.32 & 0.48 & 2.23 & 2.27 & 2.25 & 1.79 & 0.90 & 50 \\
\hline 5.12 & 2.08 & 7.92 & 7.97 & 7.95 & 0.63 & 0.38 & 40 \\
\hline
\end{tabular}

However, it appears to be very sensitive to the $\epsilon$ value and this may make any type of automation difficult. 


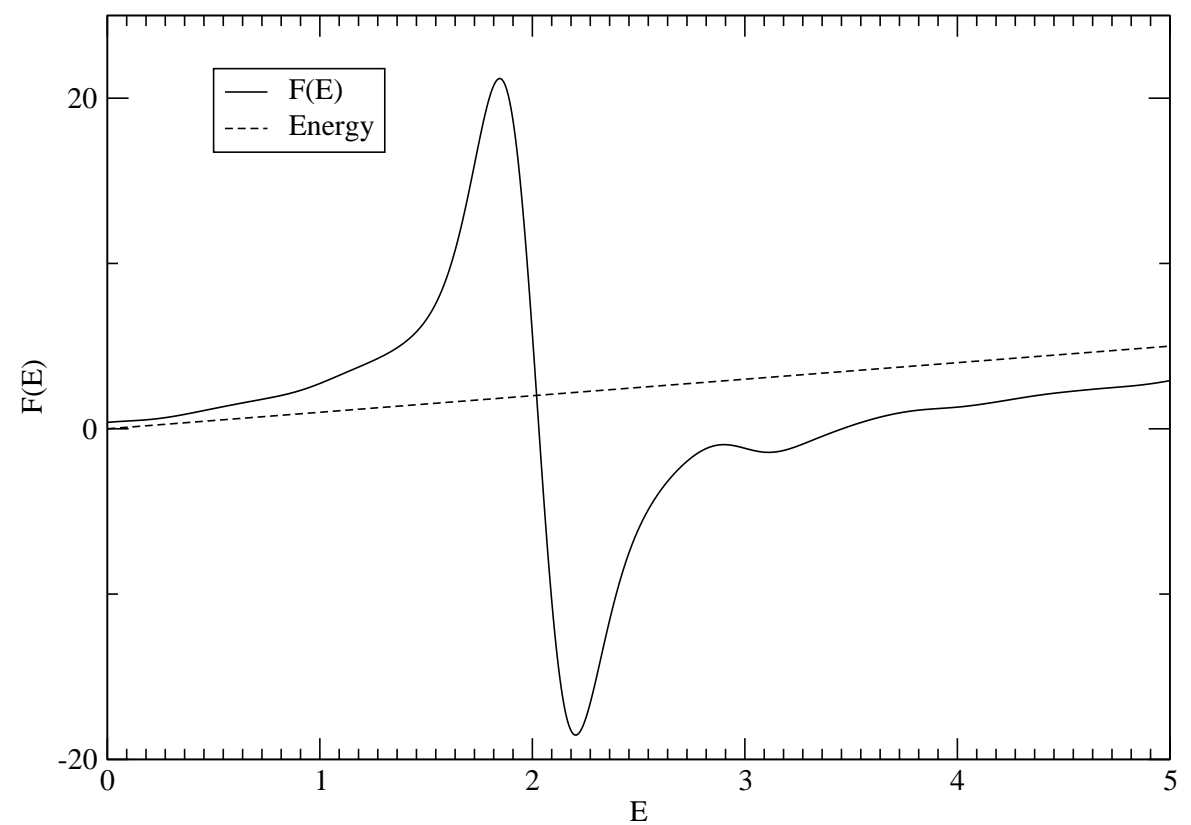

Figure 3.9: Energy intersection for Harmonium Atom with $\mathrm{k}=0.25$ and $\epsilon=0.001$ 


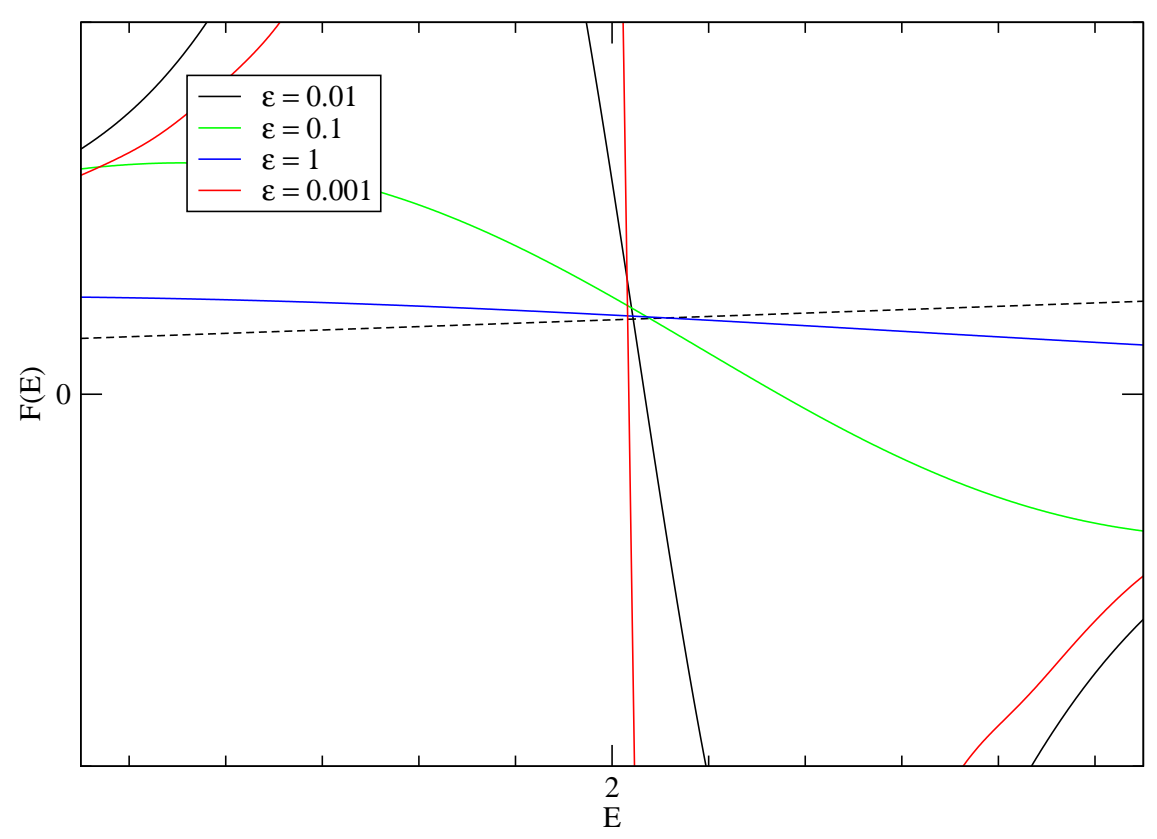

Figure 3.10: Harmonium atom of Figure 3.9 zoomed in.

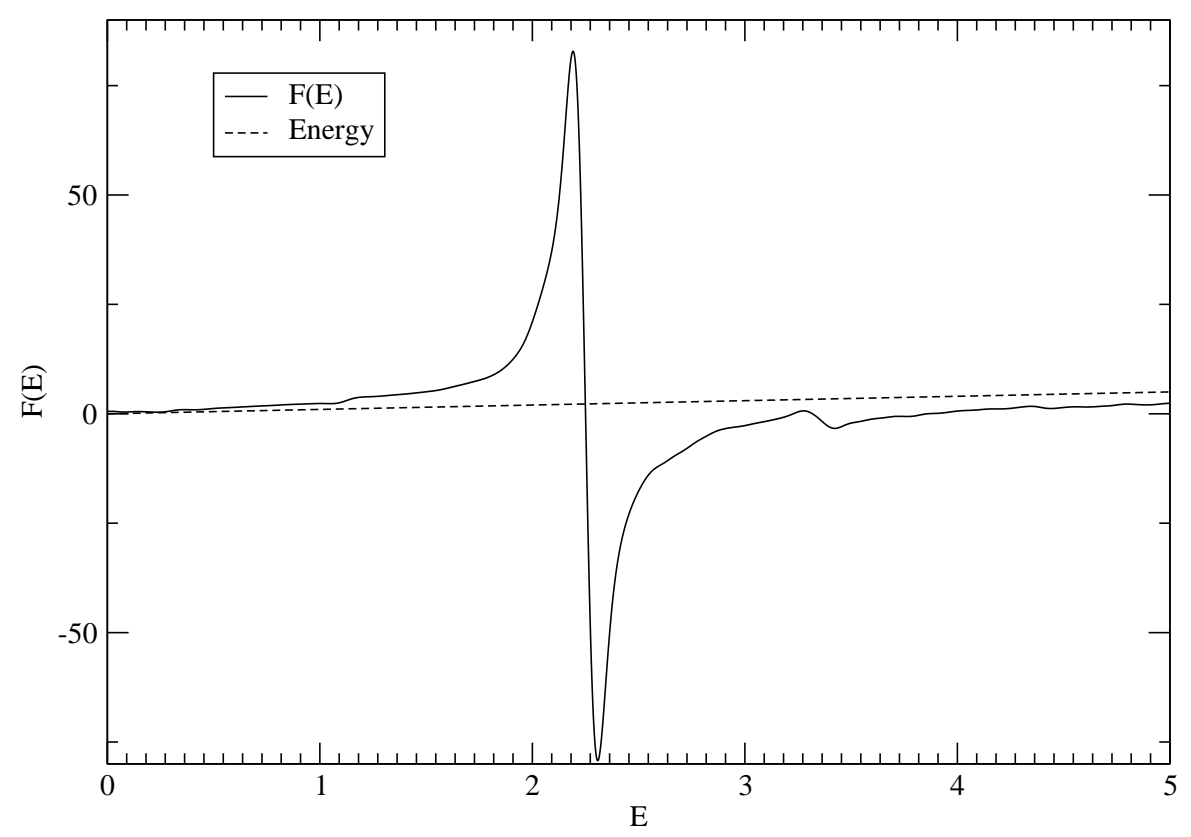

Figure 3.11: Energy intersection for Harmonium Atom with $\mathrm{k}=0.32$ and $\epsilon=0.001$ 


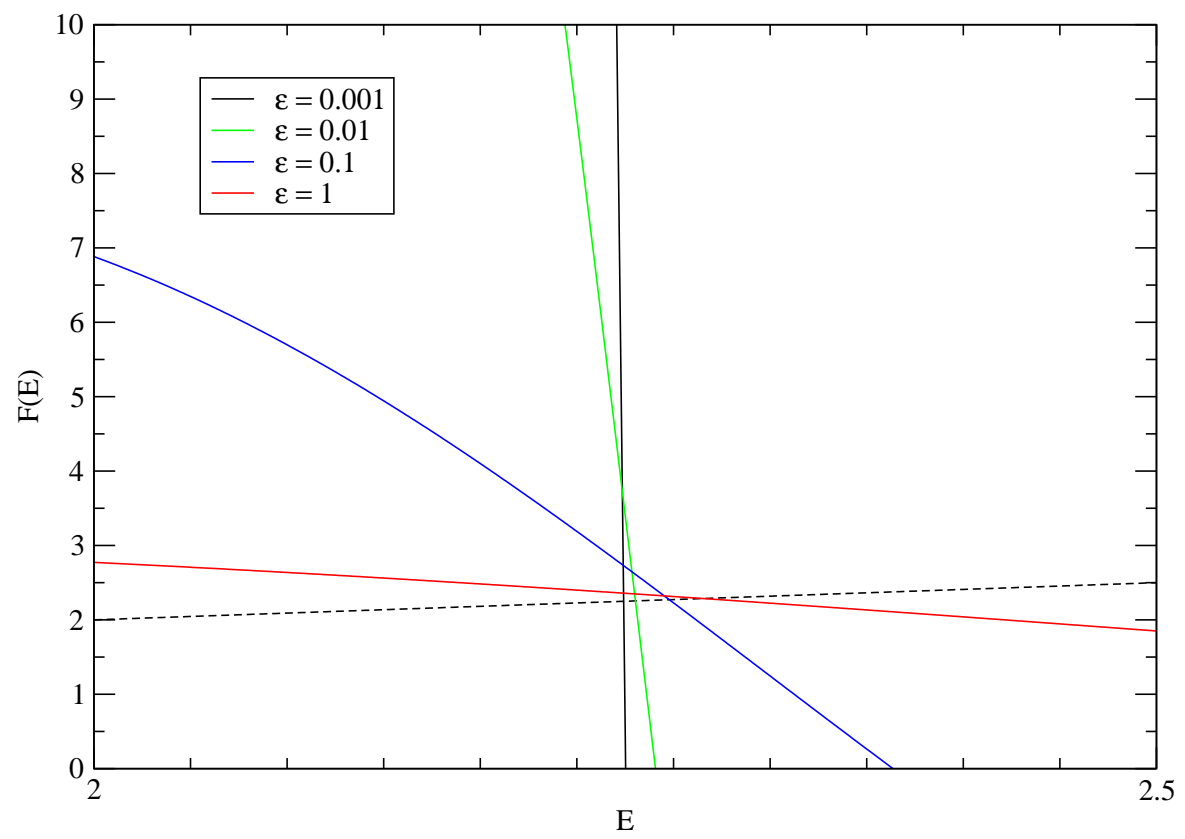

Figure 3.12: Harmonium atom of Figure 3.11 zoomed in. 


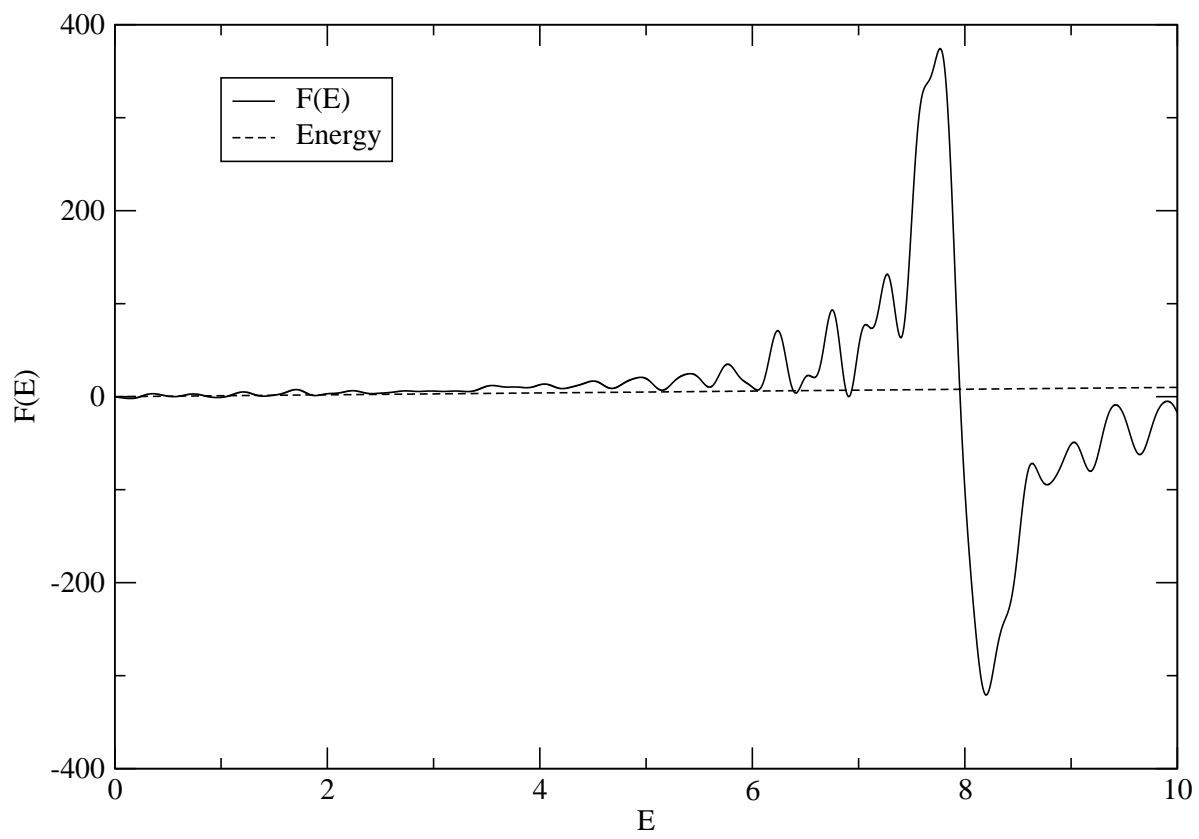

Figure 3.13: Energy intersection for Harmonium Atom with $\mathrm{k}=5.1$ and $\epsilon=0.01$ 


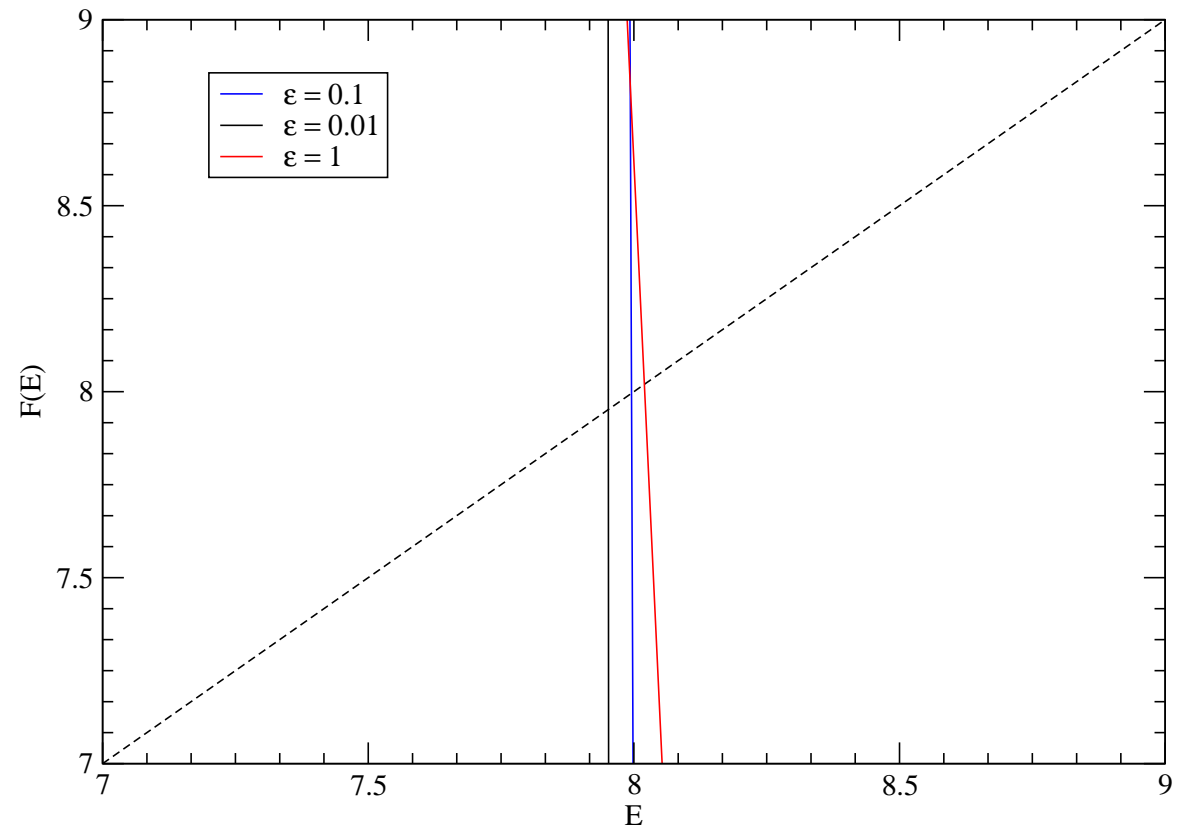

Figure 3.14: Harmonium Atom of Figure 3.13 zoomed in. 


\section{Chapter 4}

\section{Accessing Metastable States and}

\section{Long Time Dynamics - Time}

\section{Dependent Monte Carlo Sampling}

\section{Introduction}

In a previous chapter, the various form of the IVR have been described. In the most popular form, the HK-IVR the sampling function to carry out the monte carlo integration over initial trajectory conditions is the Husimi distribution. This distribution is just the modulus square of the overlap of two coherent states - the reference state with the coherent state of initial conditions -

The simulation of long time dynamics or being able to describe rare events accu- 
rately is quite a challenge to all dynamical methods. The IVR is no different as we go to longer time scales a lot of noise starts to make an appearance in, for example, a energy spectrum calculation. One way to reduce the noise is to simply run a larger and larger number of trajectories to makes sure that even the rare events are well represented in the average statistics. This solution, while effective in some case, can become far too cumbersome and expensive.

In this chapter, we describe a time dependent sampling that is a potential alternate solution to the problem of long time dynamics. This method is inspired ${ }^{55}$ by Diffusion Monte Carlo and the $\log$ derivative ${ }^{56}$ method to a certain extent. The method is applied to the problem of metastable states of ozone and also some long time water simulations. ${ }^{55}$

\section{Theory}

The Log-derivative algorithm was first used to solve multichannel scattering equations $^{57}$ and later modified and used by other groups to solve inelastic and reactive scattering problems. ${ }^{58-60}$ A log-derivative formalism for the semiclassical prefactor has also been described and we will discuss it in further detail in a later chapter 6 In the current context, the log-derivative procedure is the inspiration for the 
implementation of a time-dependent Monte Carlo sampling scheme.

Consider an autocorrelation function, with the HK-IVR expression for the propagator.

$$
A(t)=\left(\frac{1}{2 \pi \hbar}\right)^{N} \int d \mathbf{p}_{\mathbf{0}} \int d \mathbf{q}_{\mathbf{0}} C_{t}\left(\mathbf{p}_{\mathbf{0}}, \mathbf{q}_{\mathbf{0}}\right) e^{i S_{t}\left(\mathbf{p}_{\mathbf{0}}, \mathbf{q}_{\mathbf{0}}\right) / \hbar}\left\langle\psi_{i} \mid \mathbf{p}_{\mathbf{t}}, \mathbf{q}_{\mathbf{t}}\right\rangle\left\langle\mathbf{p}_{\mathbf{0}}, \mathbf{q}_{\mathbf{0}} \mid \psi_{i}\right\rangle
$$

The standard sampling function is the time-independent Husimi distribution $w_{H}$ described already in Eq. (2.2). In this section we refer to this as our zero-time sampling function.

$$
w_{0}\left(\mathbf{p}_{\mathbf{0}}, \mathbf{q}_{\mathbf{0}}\right)=\left\langle\psi_{i} \mid \mathbf{p}_{\mathbf{0}}, \mathbf{q}_{\mathbf{0}}\right\rangle\left\langle\mathbf{p}_{\mathbf{0}}, \mathbf{q}_{\mathbf{0}} \mid \psi_{i}\right\rangle
$$

The integral can now be represented as

$$
A(t)=N_{0}\left\langle A_{e}\left(\mathbf{p}_{\mathbf{0}}, \mathbf{q}_{\mathbf{0}} ; t\right)\right\rangle_{w_{0}\left(\mathbf{p}_{\mathbf{0}}, \mathbf{q}_{\mathbf{0}}\right)}
$$

where

$$
A_{e}\left(\mathbf{p}_{\mathbf{0}}, \mathbf{q}_{\mathbf{0}} ; t\right)=\left(\frac{1}{2 \pi \hbar}\right)^{N} C_{t}\left(\mathbf{p}_{\mathbf{0}}, \mathbf{q}_{\mathbf{0}}\right) e^{i S_{t}\left(\mathbf{p}_{\mathbf{0}}, \mathbf{q}_{\mathbf{0}}\right) / \hbar} \frac{\left\langle\psi_{i} \mid \mathbf{p}_{\mathbf{t}}, \mathbf{q}_{\mathbf{t}}\right\rangle}{\left\langle\mathbf{p}_{\mathbf{0}}, \mathbf{q}_{\mathbf{0}} \mid \psi_{i}\right\rangle}
$$

and the normalization constant is

$$
N_{0}=\int d \mathbf{p}_{\mathbf{0}} \int d \mathbf{q}_{\mathbf{0}} w_{0}\left(\mathbf{p}_{\mathbf{0}}, \mathbf{q}_{\mathbf{0}}\right)
$$

The Monte Carlo estimator here $A_{e}$ is the oscillatory term that is at the center of the 'sign' problem and leads to slow convergence of the integrand especially at longer times.

The idea here is that the region of phase space that is most important is constantly changing as the system evolves in time. We propose the use of a time dependent 
sampling function ${ }^{55}$ It is usually easier to use a sampling functions that is real and positive, so keeping this in mind, we choose suitable parts of the integrand.

We assume here that the reference state $\psi_{i}$ is of the form

$$
\left\langle\mathbf{q} \mid \psi_{i}\right\rangle=\left\{\frac{\gamma_{i}}{\pi}\right\}^{1 / 4} e^{-\frac{\gamma_{i}}{2}\left(\mathbf{q}-\mathbf{q}_{\mathbf{i}}\right)^{2}}
$$

We now look at the product of the overlap of the reference state with the initial coherent state and its overlap with the final coherent state at time $t$.

$$
\begin{aligned}
\left\langle\psi_{i} \mid \mathbf{p}_{\mathbf{t}}, \mathbf{q}_{\mathbf{t}}\right\rangle\left\langle\mathbf{p}_{\mathbf{0}}, \mathbf{q}_{\mathbf{0}} \mid \psi_{i}\right\rangle= & \left(\gamma_{i} \gamma\right)^{\frac{1}{2}}\left(\frac{2}{\gamma_{i}+\gamma}\right) e^{-\frac{\gamma_{i} \gamma}{2\left(\gamma_{i}+\gamma\right)}\left(\mathbf{q}_{\mathbf{0}}-\mathbf{q}_{\mathbf{i}}\right)^{2}} \\
& e^{-\frac{1}{2 \hbar^{2}\left(\gamma_{i}+\gamma\right)} \mathbf{p}^{2}-\frac{\gamma_{i} \gamma}{2\left(\gamma_{i}+\gamma\right)}\left(\mathbf{q}_{\mathbf{t}}-\mathbf{q}_{\mathbf{i}}\right)^{2}-\frac{1}{2 \hbar^{2}\left(\gamma_{i}+\gamma\right)} \mathbf{p}^{2}} e^{i \phi_{t}} \\
= & w_{t}\left(\mathbf{p}_{\mathbf{0}}, \mathbf{q}_{\mathbf{0}} ; t\right) e^{i \phi}
\end{aligned}
$$

The function $w_{t}$ is thus the time dependent sampling function. The phase of the above expression is grouped with the rest of the integrand and is of the form

$$
\phi_{t}\left(\mathbf{p}_{\mathbf{0}}, \mathbf{q}_{\mathbf{0}}\right)=\frac{\gamma_{i}}{\hbar\left(\gamma_{i}+\gamma\right)}\left[-\mathbf{p}_{\mathbf{t}} \cdot\left(\mathbf{q}_{\mathbf{t}}-\mathbf{q}_{\mathbf{i}}\right)+\mathbf{p}_{\mathbf{0}} \cdot\left(\mathbf{q}_{\mathbf{0}}-\mathbf{q}_{\mathbf{i}}\right)\right]
$$

The need for such a time dependent sampling function becomes clear in cases where the overlap between the sampling function $w_{0}$ and the one at time $t, w_{t}$ is minimal. This clearly will lead to poor sampling at long times and makes it proportionally hard to obtain a converged result for the integrand.

We are now faced with two practical questions in the implementation of the sampling function defined in Eq. (2.7). First, we need to be able to normalize the 
function $w_{t}$ before it can be used as a sampling function in the integration.

$$
N(t)=\int d \mathbf{p}_{\mathbf{0}} \int d \mathbf{q}_{\mathbf{0}} w_{t}\left(\mathbf{p}_{\mathbf{0}}, \mathbf{q}_{\mathbf{0}}\right)
$$

Second, an efficient scheme to implement this potentially problematic time dependent sampling must be described.

\section{II.1 The Normalization Constant}

The normalization constant here is

$$
N(t)=\int d \mathbf{q}_{\mathbf{0}} \int d \mathbf{p}_{\mathbf{0}} w_{t}\left(\mathbf{p}_{\mathbf{0}}, \mathbf{q}_{\mathbf{0}}\right)
$$

Clearly, this is a non-trivial integration since $w_{t}$ is a function of time evolved momentum and position $\left(\mathbf{p}_{\mathbf{t}}, \mathbf{q}_{\mathbf{t}}\right)$.

Using a log-derivative type methodology here seems to be the right approach. One way is to evaluate the correlation function itself in this manner.

$$
\frac{\dot{A(t)}}{A(t)}=\frac{\left\langle\dot{A}_{e}\left(\mathbf{p}_{\mathbf{0}}, \mathbf{q}_{\mathbf{0}} ; t\right)\right\rangle_{w_{t}}}{\left\langle A_{e}\left(\mathbf{p}_{\mathbf{0}}, \mathbf{q}_{\mathbf{0}} ; t\right)\right\rangle_{w_{t}}}
$$

The angular brackets indicate the estimator and the subscript to the brackets defines the sampling function which is used to Monte Carlo integrate over the estimator.

Since the LHS of Eq. (2.11) is the time derivative of the log of the correlation function, the solution for $A(t)$ is given by,

$$
A(t)=A(0) e^{\int_{0}^{t} d t^{\prime} \frac{\dot{A}\left(t^{\prime}\right)}{A\left(t^{\prime}\right)}}
$$


The drawback with taking this approach is that both $A(t)$ and $\dot{A}(t)$ are complicated, involving the evaluation of Monodromy matrices.

The other way to approach this problem is to evaluate the Normalization constant using the log-derivative method. This gives us our sampling function at different times.

$$
\dot{N}(t)=\int d \mathbf{p}_{\mathbf{0}} \int d \mathbf{q}_{\mathbf{0}} \dot{w}_{t}\left(\mathbf{p}_{\mathbf{0}}, \mathbf{q}_{\mathbf{0}}\right)
$$

where, from Eq. (2.7) we can easily see that

$$
\begin{aligned}
\dot{w}_{t}\left(\mathbf{p}_{\mathbf{0}}, \mathbf{q}_{\mathbf{0}}\right) & =\left[-\frac{\gamma_{i} \gamma}{m\left(\gamma_{i}+\gamma\right)} \mathbf{p}_{\mathbf{t}} \cdot\left(\mathbf{q}_{\mathbf{t}}-\mathbf{q}_{\mathbf{i}}\right)+\left(\frac{1}{\gamma_{i}+\gamma}\right) \frac{\partial V}{\partial \mathbf{q}_{\mathbf{t}}} \cdot \mathbf{p}_{\mathbf{t}}\right] w_{t}\left(\mathbf{p}_{\mathbf{0}}, \mathbf{q}_{\mathbf{0}}\right) \\
& =f_{t}\left(\mathbf{p}_{\mathbf{0}}, \mathbf{q}_{\mathbf{0}}\right) \cdot w_{t}\left(\mathbf{p}_{\mathbf{0}}, \mathbf{q}_{\mathbf{0}}\right)
\end{aligned}
$$

From Eq. (2.14) and Eq. (2.10), we now have

$$
\dot{N}(t)=\int d \mathbf{p}_{\mathbf{0}} \int d \mathbf{q}_{\mathbf{0}} f_{t} w_{t}
$$

This means that the $\frac{\dot{N}(t)}{N(t)}$ using Eq. (2.15) and Eq. (2.10)

$$
\frac{\dot{N}(t)}{N(t)}=\left\langle f_{t}\right\rangle_{w_{t}}
$$

It is possible to obtain the normalization constant from Eq. (2.16) from the fact that the left hand side is the time derivative of the log of the normalization constant.

$$
N(t)=(2 \pi \hbar)^{N / 2} e^{\int_{0}^{t} \frac{\dot{N}\left(t^{\prime}\right)}{N\left(t^{\prime}\right)} d t^{\prime}}
$$




\section{II.2 The Sampling Function}

The actual sampling function in its normalized form is

$$
\rho_{t} \mathbf{p}_{\mathbf{0}}, \mathbf{q}_{\mathbf{0}}=\frac{w_{t}\left(\mathbf{p}_{\mathbf{0}}, \mathbf{q}_{\mathbf{0}}\right)}{N_{t}\left(\mathbf{p}_{\mathbf{0}}, \mathbf{q}_{\mathbf{0}}\right)}
$$

The fact that our sampling function changes in time, is just another way of saying that the we wish to vary the importance of the different phase space points generated at time zero.

We look closely at the way this sampling function evolves in time.

$$
\begin{aligned}
\dot{\rho}_{t} & =\left(\frac{\dot{w}_{t}}{w_{t}}-\frac{\dot{N}_{t}}{N_{t}}\right) \rho_{t} \\
& =\left(f_{t}-\frac{\dot{N}_{t}}{N_{t}}\right) \rho_{t}
\end{aligned}
$$

This equation resembles very closely the Diffusion Monte Carlo equation, which describes the time evolution of the wavefunction as,

$$
\frac{\partial \psi(x)}{\partial s}=\left[D \nabla-\left(V(x)-E_{0}\right)\right] \psi(x)
$$

The kinetic part of this equation is described by the diffusion process and the potential part by the auto-catalysis process.

In drawing the parallel between Eq. (2.19) and Eq. (2.20) it becomes clear that the sampling function does not have a diffusion term. This means that once the initial ensemble $\left(\mathbf{p}_{\mathbf{0}}, \mathbf{q}_{\mathbf{0}}\right)$ is generated as per the sampling function $\rho_{0}\left(\mathbf{p}_{\mathbf{0}}, \mathbf{q}_{\mathbf{0}}\right)$. The actual set of points remains unchanged, but the population of trajectories at each of these points does change 
We further note that in Eq. (2.19) while we know the function $f_{t}$, the remaining

part of the equation $\frac{\dot{N}(t)}{N(t)}$ is not known apriori. If we use just $f_{t}$ to evolve the equation, the number of trajectories in no longer preserved and will be different for different times. In order to conserve the number of trajectories we follow a two step process.

First, we do a rough trajectory evolution from 0 to time $t$ and calculate the normalization constant from this run. This leads to our second step, which is to now do a full scale calculation of the correlation function with Monodromy matrices thrown in as well. At every time step (these can be pretty far apart and need not correspond to the trajectory time steps), we can use an appropriate function to describe the relative importance of the various phase space points, which in turn determine how many trajectories contribute from each point of the initial ensemble and which points do not contribute at all.

\section{II.3 The Sampling Algorithm}

The sampling function evolves in time according to Eq. (2.19). The expression for $\rho(t)$ becomes

$$
\rho(t)=\rho(0) e^{\int_{0}^{t}\left(f_{t^{\prime}}-\frac{\dot{N}\left(t^{\prime}\right)}{N\left(t^{\prime}\right)} d t^{\prime}\right.}
$$

As stated before, $f_{t}$ is known. We do a preliminary run to determine this part of the equation and then use it to weight the individual phase space points and 
decide which regions are to be made more important and which ones less. We wish to follow these changes as best we can, without having the calculation become too tedious. As discussed before, there is no 'diffusion' term so we introduce an artificial shake term time that allows us to generate an extra point in an 'important' neighborhood while eliminating points that are in less important regions thereby preserving the total number of points in time.

To make this clearer, we describe a sampling algorithm that it carried out after the first purely classical run.

Step 1 :

At the $i^{\text {th }}$ time step, for each point $\left(\mathbf{p}_{\mathbf{0}}, \mathbf{q}_{\mathbf{0}}\right)$ in the sampling set we evaluate an importance function of the form

$$
K\left(\mathbf{p}_{\mathbf{0}}, \mathbf{q}_{\mathbf{0}}\right)=e^{\left(\left\langle f_{t}\right\rangle_{w_{t}}\right)}
$$

Step 2:

In this step we wish to translate the importance of a point, into an actual increase in the number of points in its neighborhood that will contribute to the integrand. The number of points that should be in this region is calculated as $\operatorname{int}(K-1)$ where we subtract one to account for the existing point. We note here that $\operatorname{int}(K)$ gives us the nearest smaller integer. 
The probability of increasing the number of points in this vicinity is then,

$$
u=K-\operatorname{int}(K)
$$

If we are increasing the number of points, we also need a probability of eliminating points. In this case, it is clear that we wish to eliminate points where $K<1$ with a probability of $1-K$.

Step 3 : Since we have already calculated the normalization factor, we now use this set of points $\left(\mathbf{p}_{\mathbf{0}}, \mathbf{q}_{\mathbf{0}}\right)$ and do a regular Monte Carlo walk around each of these with $w_{t}$ as the weight function.

We then repeat these steps for as many time steps as we are looking at. This calculation can be carried out a coarser grid than we would normally do, since the time steps where we stop and evaluate the correlation function can typically be bigger than the trajectory time steps.

\section{Applications}

\section{III.1 1D Ozone}

The Ozone potential we model is an 1D PES which was developed to study the energy levels of metastable states of ozone near dissociation. ${ }^{61}$ The potential is of the form,

$$
V(r)=V_{0}\left(1-e^{-\alpha\left(r-r_{0}\right)}\right)^{2}+\frac{\hbar^{2} J(J+1)}{2 \mu r^{2}}
$$


The first part of the potential is a simple Morse potential and the second term is a centrifugal term where we can choose the angular momentum level to be studied. In this case, we use the parameters for

$$
\begin{aligned}
V_{0} & =1.132 \mathrm{eV} \\
\alpha & =2 a . u . \\
r_{0} & =2.4 a . u .
\end{aligned}
$$

The initial state is chosen to be a gaussian centered at the top of the dissociation barrier with $x_{0}=4.64$ a.u. and $\gamma=4$

\section{III.2 Water}

A 3D test of this method was carried out by Yimin $^{55}$ with a model of the water molecule in normal coordinates. The water potential is a PJT2 potential developed by Polyansky et al. ${ }^{62}$

\section{Discussion}

We have already stated that the reason time dependent sampling is necessary is because as the sample set evolves in time, we find that it is restricting to use the zero time sampling function and very often the overlap of the important regions at time $t$ and time zero is not good. In a series of Figures 4.1(a) -4.1(f) we show importance maps of the sampling function. It becomes clear that the important 
regions are spreading - in other words a single gaussian centered at initial conditions is no longer sufficient.

The figures show the most important regions in red and as time progresses we can see how this one two-dimensional gaussian region splits into several different regions of importance. The results of the autocorrelation function for the $1 \mathrm{D}$ ozone potential are plotted in figures 4.1 and 4.2 which contain the real and imaginary parts of the function respectively We see that the HK-IVR with regular sampling does quite well in this case, however at longer times it takes fewer trajectories to converge our time dependent sampling IVR. In the results shown, 360000 trajectories were used to achieve the regular result, where only 3600 were required for our technique.

We also show in Figure 4.3 the exact and calculated (by log derivative) normalization constant for this example and show that there is a good agreement between these two results. Similarly for the water model the normalization constant is shown in Figure 4.4 and the real and imaginary parts of the autocorrelation function are shown in Figure 4.5 and 4.6 respectively. We see that although there is not much to choose between the two methods initially, but at longer times it becomes evident that time dependent sampling is far more efficient. Also, equally importantly, we used much fewer trajectories for the time dependent method - 50,000 as opposed to 500,000 for the regular sampling. 


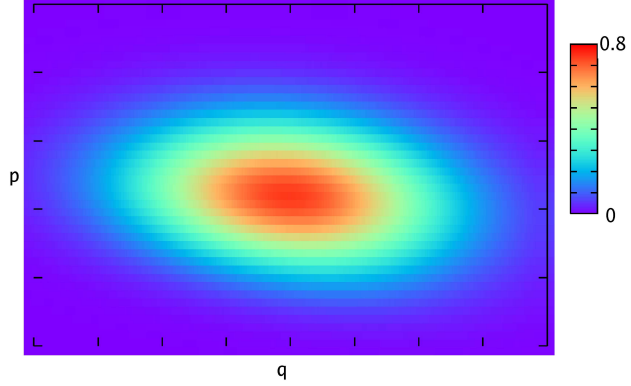

(a) Sampling function at time 0 a.u

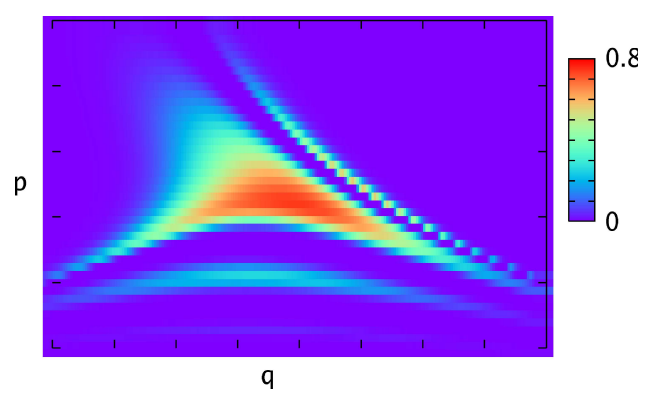

(c) Sampling function at time 30 a.u.

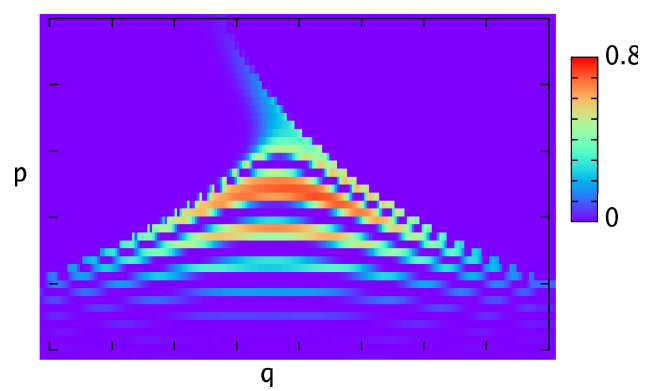

(e) Sampling function at time 70 a.u.

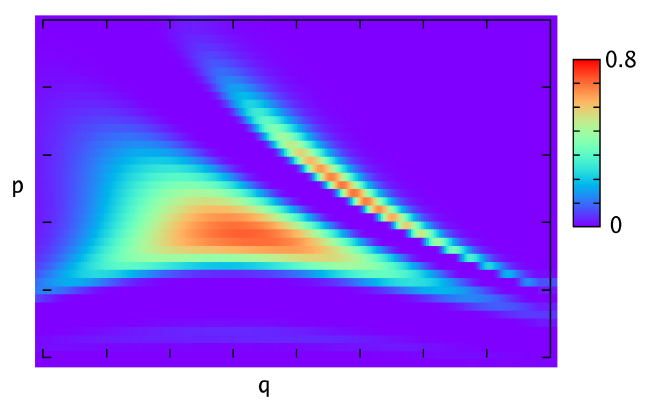

(b) Sampling function at time 20 a.u.

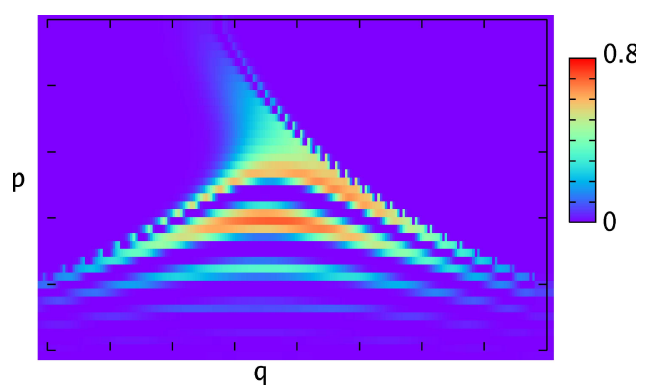

(d) Sampling function at time 50 a.u.

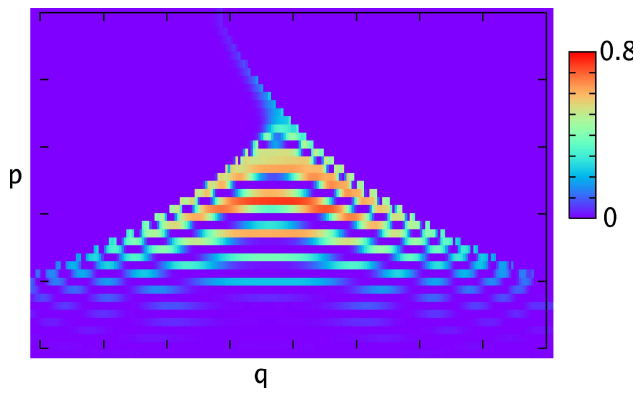

(f) Sampling function at time 90 a.u. 


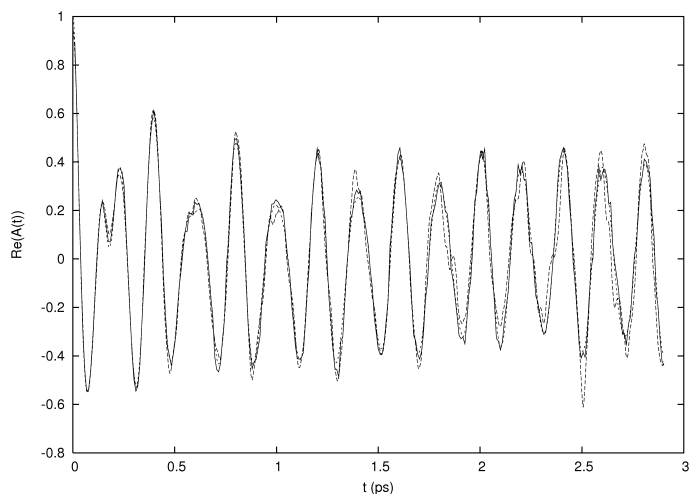

Figure 4.1: Real part of autocorrelation function for Ozone. The dashed line represents the regular SC-IVR calculation and the time dependent sampling method is the full line

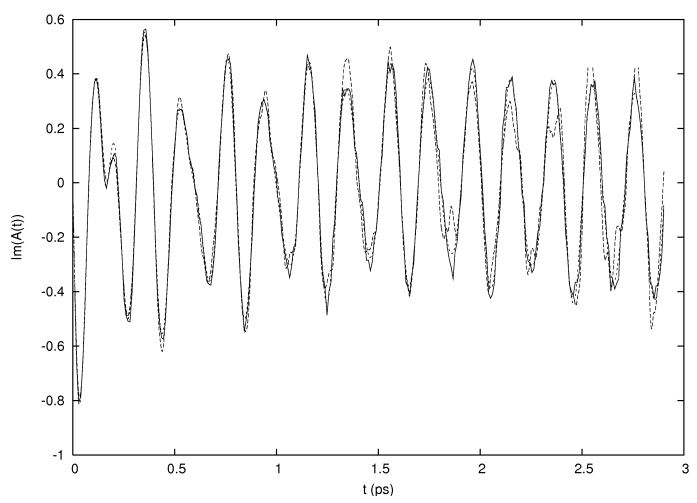

Figure 4.2: Imaginary part of autocorrelation function for Ozone. The dashed line represents the regular SC-IVR calculation and the time dependent sampling method is the full line 


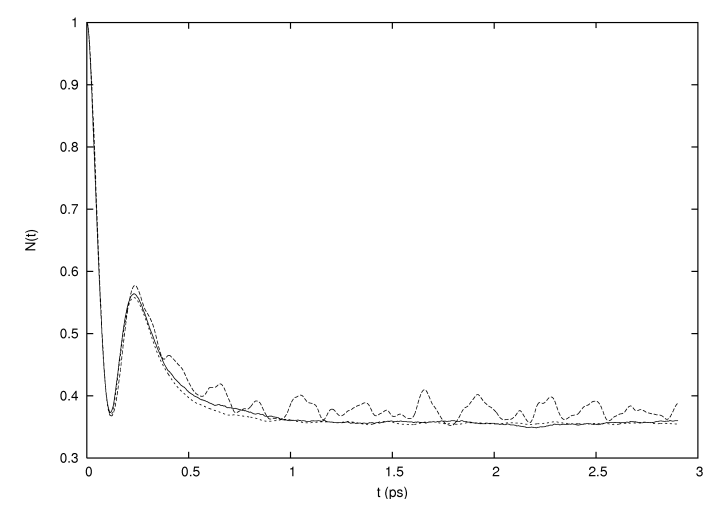

Figure 4.3: The Normalization constant is obtained exactly (full line) or with the Log-Derivative method.

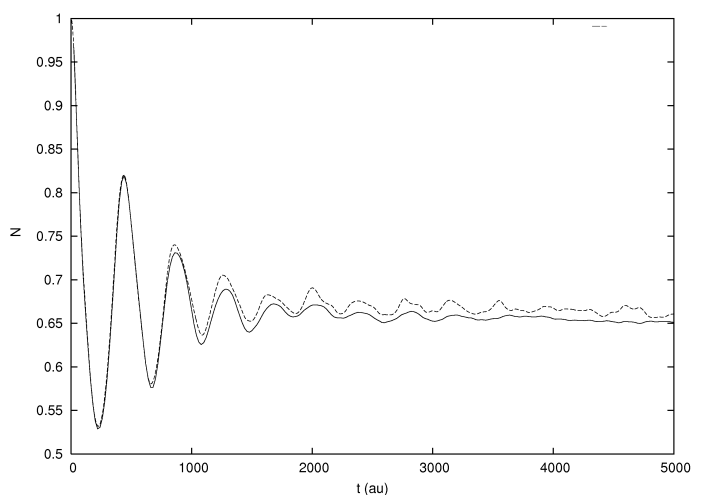

Figure 4.4: The Normalization constant is obtained exactly (full line) or with the Log-Derivative method (dashed line). 


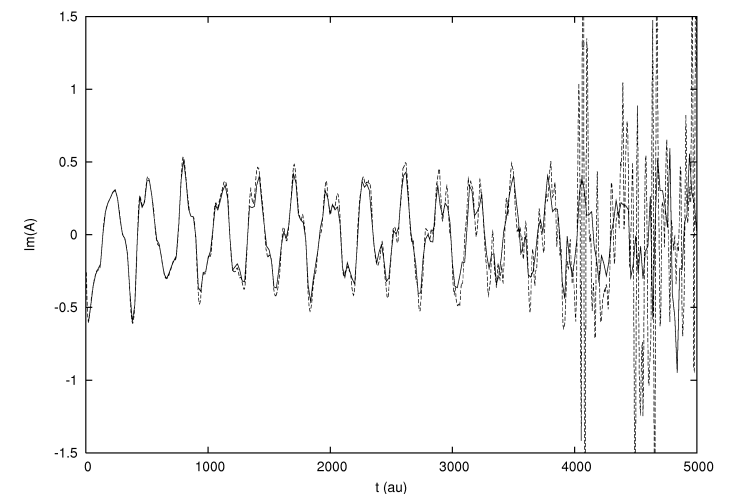

Figure 4.5: Imaginary part of the autocorrelation function for the 3D model of water. The dashed line is the regular sampling method and the full line is time dependent sampling.

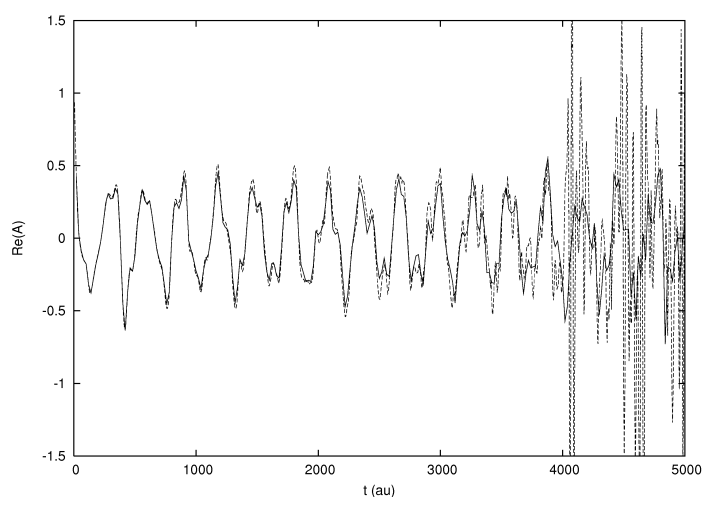

Figure 4.6: Real part of autocorrelation function for the 3D model of water. The dashed line is the regular sampling method and the full line is the time dependent sampling. 


\section{Chapter 5}

\section{Semiclassical Approach to}

\section{Nonadiabatic Dynamics}

\section{Introduction}

Classical molecular dynamics (MD) simulations are the most common and generally applicable way to describe dynamical processes in large molecular systems. When electronically non-adiabatic processes are involved (those that involve transitions between different Born-Oppenheimer (BO) potential energy surfaces) it is natural to try to generalize MD simulations to deal with them. This has led to a variety of mixed quantum-classical (QC) models, where the electronic degrees of freedom are treated quantum mechanically via an electronic wavefunction determined by the time-dependent Schroedinger equation, and the nuclear degrees 
of freedom (dof) classically as numerically computed classical trajectories. The time dependence for the electronic dof comes from the dependence of the potential energy surfaces and their couplings on the nuclear coordinates, which vary with time along classical trajectories.

The various mixed $\mathrm{QC}$ models differ primarily in the forces, or equivalently the effective potential surfaces that determine the classical motion of the nuclei. Most such approaches can be classified as either Ehrenfest (mean field) models or surfacehopping models. Tully ${ }^{63}$ has given an excellent discussion of the differences of these two approaches as well as the strengths and weaknesses of each. Briefly, in Ehrenfest models one computes the force on the nuclei by averaging the electronic potential surface (matrix) using the instantaneous electronic wave function, while in surface hopping approaches the force is determined from one (adiabatic) potential surface or another, with instantaneous hops between different adiabatic surfaces permitted according to some hopping algorithm.

A positive feature of the Ehrenfest model is that it is a well defined approximation, while surface-hopping models have a number of ad hoc features which, though physically reasonable, may be difficult to justify. A significant shortcoming of the Ehrenfest model is that the nuclear trajectories are determined by the average PES even in the asymptotic region of a scattering problem, where the nuclear trajectories should, of course, be determined by one PES or another depending on the electronic state. The mean field aspect of the Ehrenfest model is not able to 
describe this correlation.

A significant contribution to the theory was made by Neria and Nitzan ${ }^{64}$ in pointing out that the failure of these mixed QC models results from the fact that the nuclear dof are not described by a wavefunction, but only as classical trajectories. Though their analysis was based only on the Golden Rule (lowest order perturbation theory), it correctly identified the problem and suggested some ways to try to overcome it, for instance, by assigning a Gaussian wavepacket (nuclear wavefunction) to the classical trajectories of the nuclei. Rossky et al ${ }^{65}$ picked up on this idea and made good use of it, and more recently Truhlar et al ${ }^{66}$ have also used it to construct more refined surface hopping models. (Both of these groups have focused on determining a decoherence times for the nuclear wavefunction to collapse to one electronic PES or another.)

The initial value representation (IVR) of semiclassical (SC) theory ${ }^{20,36}$ provides a natural and correct description of electronically non-adiabatic processes. This is perhaps not surprising since the nuclear (and electronic) dof do have a wavefunction in the SC description. It is interesting, though, that the equations of motion that determine the nuclear trajectories are precisely the Ehrenfest equations, but by processing the trajectories though $\mathrm{SC}$ theory, the nuclear motion emerges (in a scattering problem, as the present examples are) on one PES or another, not on the average PES. 
To apply SC theory to a vibronic system (one with electronic and nuclear dof), we use the Meyer-Miller-Stock-Thoss(MM-ST) classical analog model for the electronic degrees of freedom. This replaces the $\mathrm{N}$ electronic states involved in the process by $\mathrm{N}$ classical degrees of freedom, specifically a harmonic oscillator for each electronic state. All dof - those of the nuclei and those of the N 'electronic' oscillators - are then treated semiclassically via the IVR methodology (involving trajectories computed in the full coordinate and momentum space of nuclear and 'electronic' dof). Though $\mathrm{MM}^{67}$ developed this classical model for the electronic degrees of freedom as an approximate way to be able to treat electronic and nuclear dynamics on a equal footing, the more recent derivation of the model by Stock and Thoss $(\mathrm{ST})^{68}$ shows that it is actually not a model (i.e approximation), but rather an exact representation of the full vibronic system. In other words, if one were to take the MM-ST vibronic Hamiltonian and implement it quantum mechanically, the exact vibronic dynamics would be obtained. The only approximation here is that it is implemented semiclassically via the IVR.

It has been previously shown that ${ }^{69}$ the approach described above captured transmission probabilities accurately for Tully's ${ }^{63}$ three model systems. In this paper we further show that the IVR and some of its variations can indeed provide a complete dynamical picture of nonadiabatic systems both in terms of instantaneous populations of states as well as the quantum coherences between them. In the theory section, we first gives a brief summary of the Ehrenfest method, followed 
with an introduction to the MM-ST description of the electronic-nuclear system, We utilize the Forward-Backward(FB) version of the IVR approach, which makes the calculations efficient and yet retains the ability to describe quantum coherence effects correctly. As seen in the examples treated in Section IV, if one makes the cruder linearized approximation to the SC-IVR (which reduces to the classical Wigner model), one loses the ability to describe coherence; the results of this linearized, or LSC-IVR approximation become essentially those of the traditional Ehrenfest model, with the nuclear trajectory emerging (incorrectly) on the average of the electronic PES's. With the more rigorous FB-IVR, however, the nuclear trajectory emerges on one potential energy surface or the other.

\section{Theory}

\section{II.1 The Ehrenfest Method}

The Ehrenfest method takes a mean field approach to nuclear time evolution. It is assumed that we can separate the wavefunction into a fast moving electronic part and a slow-moving nuclear part.

$$
\psi(\mathbf{r}, \mathbf{R} ; t)=\psi_{1}(\mathbf{r} ; t) . \psi_{2}(\mathbf{R} ; t)
$$

We can now write down the time dependent Schroödinger equation for the indi- 
vidual wavefunctions as

$$
\begin{aligned}
{\left[-\frac{\hbar^{2}}{2 \mu_{r}} \nabla_{\mathbf{r}}^{2}+V_{1}^{e f f}(\mathbf{r} ; t)\right] \psi_{1}(\mathbf{r}, t) } & =i \hbar \frac{\partial \psi_{1}(\mathbf{r} ; t)}{\partial t} \\
{\left[-\frac{\hbar^{2}}{2 \mu_{R}} \nabla_{\mathbf{R}}^{2}+V_{2}^{e f f}(\mathbf{R} ; t)\right] \psi_{2}(\mathbf{R}, t) } & =i \hbar \frac{\partial \psi_{2}(\mathbf{R} ; t)}{\partial t}
\end{aligned}
$$

The effective potential energy surfaces are average fields integrated over either $\mathbf{r}$ or $\mathbf{R}$ depending on which one we are looking at.

$$
\begin{aligned}
V_{1}^{e f f}(\mathbf{r} ; t) & =\left\langle\psi_{2}(\mathbf{R} ; t)|V(\mathbf{r}, \mathbf{R})| \psi_{2}(\mathbf{R} ; t)\right\rangle_{R} \\
V_{2}^{e f f}(\mathbf{R} ; t) & =\left\langle\psi_{1}(\mathbf{r} ; t)|V(\mathbf{r}, \mathbf{R})| \psi_{1}(\mathbf{r} ; t)\right\rangle_{r}
\end{aligned}
$$

The nuclei here are taken to move along a classical trajectory, so we assume that the coordinates $\mathbf{R}$ and their corresponding momenta $\mathbf{P}$ obey Hamilton's equations of motion. We therefore have,

$$
\begin{aligned}
\dot{\mathbf{P}} & =-\frac{\partial V_{2}^{e f f}}{\partial \mathbf{R}} \\
\dot{\mathbf{R}} & =\frac{\mathbf{P}}{\mu_{R}}
\end{aligned}
$$

The effective potential energy for the fast moving part of the system is then obtained as an average at each time over the weighted classical trajectories

$$
V_{1}^{e f f}(\mathbf{r} ; t)=\frac{1}{N_{t}} \sum_{\alpha=1}^{N_{t}} V\left(\mathbf{r}, \mathbf{R}_{\alpha}(t)\right)
$$

The method ensures that each subsystem feels an averaged out force due to the other and thus describes a 'mean-field' path for our system to evolve along in time. The average path while useful in some situations describes all properties averaged 
to a single probability and cannot therefore, describe multiple state probabilities that we expect from Quantum Mechanics.

\section{II.2 The Meyer-Miller-Stock-Thoss (MMST) Hamiltonian}

$\mathrm{MM}^{67}$ arrived at their classical model for electronic degrees of freedom in the following way. If $V_{k k^{\prime}}(\mathbf{R}(t)), k, k^{\prime}=1, \ldots N$ is the $\mathrm{NxN}$ diabatic electronic potential matrix for $\mathrm{N}$ electronic states, where the nuclear coordinates follow some given trajectory $\mathbf{R}(t)$, and the electronic wavefunction is given by an expansion in terms of the $\mathrm{N}$ diabatic electronic states,

$$
\left|\Psi_{e l}(t)\right\rangle=\sum_{k=1}^{N}\left|\phi_{k}\right\rangle c_{k}(t),
$$

then the time-dependent Schroedinger equation for the electronic amplitudes reads $($ with $\hbar=1)$

$$
i \dot{c_{k}}(t)=\sum_{k^{\prime}=1}^{N} V_{k k^{\prime}}(\mathbf{R}(t)) c_{k^{\prime}}(t)
$$

MM noted that if the complex-valued electronic amplitudes $c_{k}(t)$ were written in terms of a magnitude and phase,

$$
c_{k}=\sqrt{n_{k}+\frac{1}{2}} e^{-i q_{k}}
$$

and $\left(n_{k}, q_{k}\right)$ considered to be canonically conjugate action-angle variables (generalized coordinates and momenta), then Hamilton's equations of motion for the 
electronic action-angle variables,

$$
\begin{array}{r}
\dot{q_{k}}=\frac{\partial H_{e l}(\mathbf{n}, \mathbf{q} ; t)}{\partial n_{k}} \\
\dot{n_{k}}=-\frac{\partial H_{e l}(\mathbf{n}, \mathbf{q} ; t)}{\partial q_{k}}
\end{array}
$$

are identical to the time-dependent Schrödinger equation of Eq. (2.10) if one defines the classical electronic Hamiltonian as

$$
\begin{aligned}
H_{e l}(\mathbf{n}, \mathbf{q} ; t) & =\sum_{k, k^{\prime}=1}^{N} c_{k}^{*}(t) V_{k k^{\prime}}(\mathbf{R}(t)) c_{k^{\prime}}(t) \\
& =\sum_{k=1}^{N} n_{k} V_{k k}(\mathbf{R}(t))+2 \sum_{k<k^{\prime}=1}^{N} V_{k k^{\prime}}(\mathbf{R}(t)) \sqrt{n_{k}+\frac{1}{2}} \sqrt{n_{k^{\prime}}+\frac{1}{2}} \cos \left(q_{k}-q_{k^{\prime}}\right)
\end{aligned}
$$

where the constant term $\frac{1}{2} \sum_{k=1}^{N} V_{k k}$ (which is independent of $n_{k}, q_{k}$ ) has been subtracted in obtaining Eq. (2.13). When the nuclear kinetic energy, $\mathbf{P}^{2} / 2 \mu$, is now added to the electronic Hamiltonian of Eq. (2.13), the full classical vibronic Hamiltonian is then given in terms the nuclear $(\mathbf{R}, \mathbf{P})$ and electronic $(\mathbf{n}, \mathbf{q})$ coordinates and momenta as

$$
\begin{aligned}
H(\mathbf{P}, \mathbf{R}, \mathbf{n}, \mathbf{q}) & =\frac{\mathbf{P}^{2}}{2 \mu}+\sum_{k=1}^{N} n_{k} V_{k k}(\mathbf{R}) \\
& +2 \sum_{k<k^{\prime}=1}^{N} V_{k k^{\prime}}(\mathbf{R}) \sqrt{n_{k}+\frac{1}{2}} \sqrt{n_{k^{\prime}}+\frac{1}{2}} \cos \left(q_{k}-q_{k^{\prime}}\right)
\end{aligned}
$$

MM used action-angle variables for the electronic dof because they were implementing the model within the (very crude) the semi-classical procedure, whereby the initial conditions (at $t=0$ ) for the action variables were $n_{i}(0)=1$ for the initial 
electronic state $\mathrm{i}, n_{k}(0)=0$ for all other states $k \neq i$ and with all angle variables chosen to be $2 \pi *$ random number. The final values of the action variables resulting from computing classical trajectories (in the full nuclear + electronic set of variables) with these initial conditions and this Hamiltonian were then histogrammed in the typical quasi-classical procedure.

Even though MM specified initial conditions in terms of action angle variables as noted above, they typically transformed to Cartesian 'electronic' variables to carry out the calculations (because they are simpler and better behaved numerically). Defining the electronic oscillator coordinates and momenta in the usual way,

$$
\begin{aligned}
& x_{k}=\sqrt{2 n_{k}+1} \cos \left(q_{k}\right) \\
& p_{k}=-\sqrt{2 n_{k}+1} \sin \left(q_{k}\right)
\end{aligned}
$$

the Hamiltonian of Eq. (2.14) becomes

$$
\begin{aligned}
H(\mathbf{P}, \mathbf{R}, \mathbf{p}, \mathbf{x})= & \frac{\mathbf{P}^{2}}{2 \mu}+\sum_{k=1}^{N} V_{k k}(\mathbf{R}) \frac{1}{2}\left(p_{k}^{2}+x_{k}^{2}-1\right) \\
& +\sum_{k<k^{\prime}=1}^{N} V_{k k^{\prime}}(\mathbf{R})\left(p_{k} p_{k^{\prime}}+x_{k} x_{k^{\prime}}\right)
\end{aligned}
$$

and this is precisely the Hamiltionian that $\mathrm{ST}^{68}$ obtained by a different, more rigorous procedure. Furthermore, the derivation by ST makes it clear that this model is actually an exact representation of the vibronic system so that, if the Hamiltonian of Eq. (2.16) were taken to be a Hamiltonian operator in the usual way, then exact vibronic dynamics would be obtained from the resulting Schrödinger 
equation. The only approximation is therefore the SC-IVR approximation for the propagator.

An interesting observation from this classical vibronic Hamiltonian, Eq. (2.16), concerns the force that the nuclei experience along a classical trajectory. The Newtonian version of the classical equations of motion gives,

$$
\begin{aligned}
\mu \ddot{\mathbf{R}}(t)= & -\sum_{k=1}^{N} \frac{1}{2}\left(p_{k}^{2}+x_{k}^{2}-1\right) \frac{\partial V_{k k}(\mathbf{R})}{\partial \mathbf{R}} \\
& -\sum_{k<k^{\prime}=1}^{N}\left(p_{k} p_{k^{\prime}}+x_{k} x_{k^{\prime}}\right) \frac{\partial V_{k k^{\prime}}(\mathbf{R})}{\partial \mathbf{R}},
\end{aligned}
$$

or if this is expressed in terms of the original electronic amplitudes $c_{k}$ it reads

$$
\mu \ddot{\mathbf{R}}(t)=-\sum_{k, k^{\prime}=1}^{N} c_{k}^{*} \frac{\partial V_{k k^{\prime}}(\mathbf{R})}{\partial \mathbf{R}} c_{k^{\prime}},
$$

which is recognized to be the Ehrenfest force. The traditional Ehrenfest model would choose initial conditions for the amplitudes as $c_{i}(0)=1$ for the initial state $\mathrm{i}$, and $c_{k}(0)=0$ for all other states much like the 'quasi-classical' model originally used by MM.

For the SC-IVR, however, the initial conditions for the electronic degrees of freedom are specified by the initial electronic-oscillator wavefunction in coordinate space $x_{k}$. Therefore for initial electronic state $\mathrm{i}$, the initial oscillator for that is one quantum of excitation, and all the other modes $k \neq i$ have zero quanta of excitation (corresponding to the ground state). The initial electronic-oscillator wavefunction 
for the $i^{\text {th }}$ electronic state is thus

$$
\begin{aligned}
\Phi_{i}\left(x_{1} . . x_{N}\right) & =\phi_{1}\left(x_{i}\right) \prod_{k=1, k \neq i}^{N} \phi_{0}\left(x_{k}\right) \\
& =\frac{\sqrt{(} 2)}{\pi^{N / 4}} x_{i} e^{-\frac{1}{2} \mathbf{x}^{2}},
\end{aligned}
$$

Finally, we note that the classical vibronic Hamiltonian in Eq. (2.16) is that for the diabatic electronic representation, and there is an analogous one for the adiabatic representation. In terms of Cartesian coordinates and momentum of the electronic oscillators (as in Eq. (2.16)), it is

$$
H(\mathbf{P}, \mathbf{R}, \mathbf{p}, \mathbf{x})=\sum_{k=1}^{N} \frac{1}{2}\left(p_{k}^{2}+x_{k}^{2}-1\right) E_{k}(\mathbf{R})+\frac{|\mathbf{P}+\boldsymbol{\Delta} \mathbf{P}|^{\mathbf{2}}}{2 \mu}
$$

where $E_{k}(\mathbf{R})$ are the usual adiabatic, or, Born-Oppenheimer potential energy surfaces, and $\Delta \mathbf{P}$ is given by

$$
\boldsymbol{\Delta} \mathbf{P}=\sum_{k<k^{\prime}=1}^{N} \hbar\left\langle\psi_{k} \mid \frac{\partial \psi_{k^{\prime}}}{\partial \mathbf{R}}\right\rangle\left(x_{k} p_{k^{\prime}}-x_{k^{\prime}} p_{k}\right)
$$

where the electronic matrix elements in Eq. (2.21) are the usual non-adiabatic derivative coupling.

\section{Applications}

Calculations have been carried out for model systems consisting of 2 electronic states and 1 nuclear degree of freedom (translation), the same kind of models Tully used earlier for testing various surface-hopping approaches. A diabatic electron 
matrix $V_{n n^{\prime}}(R), n, n^{\prime}=1,2$ characterizes the 2 electronic states. The overall system thus consists of 3 degrees of freedom, for which the classical vibronic Hamiltonian of Eq. (2.16) is

$$
\begin{gathered}
H\left(P, R, p_{1}, x_{1}, p_{2}, x_{2}\right)=\frac{P^{2}}{2 \mu}+V_{11}(R) \frac{1}{2}\left(p_{1}^{2}+x_{1}^{2}-1\right)+V_{22}(R) \frac{1}{2}\left(p_{2}^{2}+x_{2}^{2}-1\right) \\
+V_{12}(R)\left(p_{1} p_{2}+x_{1} x_{2}\right)
\end{gathered}
$$

Figures 5.1 and 5.2 show the diabatic (and also the adiabatic) potential functions for the two specific models that are treated; the specific forms of the diabatic electronic matrix for model 1 are

$$
\begin{aligned}
& V_{11}(R)=V_{0} \tanh (a R) \\
& V_{22}(R)=-V_{0} \tanh (a R) \\
& V_{12}(R)=C e^{-D R^{2}}
\end{aligned}
$$

and for model 2 are

$$
\begin{aligned}
& V_{11}(R)=V_{1} \tanh (a R) \\
& V_{22}(R)=-V_{2} \tanh (a R) ; \quad V_{1}>V_{2} \\
& V_{12}(R)=C e^{-D(R+f)^{2}}
\end{aligned}
$$

Model 1 is identical to one of the examples Tully used, and the parameters used here are $V_{0}=0.01, \mathrm{a}=1.6, \mathrm{C}=0.005, \mathrm{D}=1$. Model 2 is an asymmetric system where one channel has a very high energetic threshold and the parameters used in 
this case are $V_{1}=0.04, V_{2}=0.01, \mathrm{C}=0.005, \mathrm{D}=1, \mathrm{f}=0.7$.

The quantity calculated in all cases is the probability distribution of final nuclear

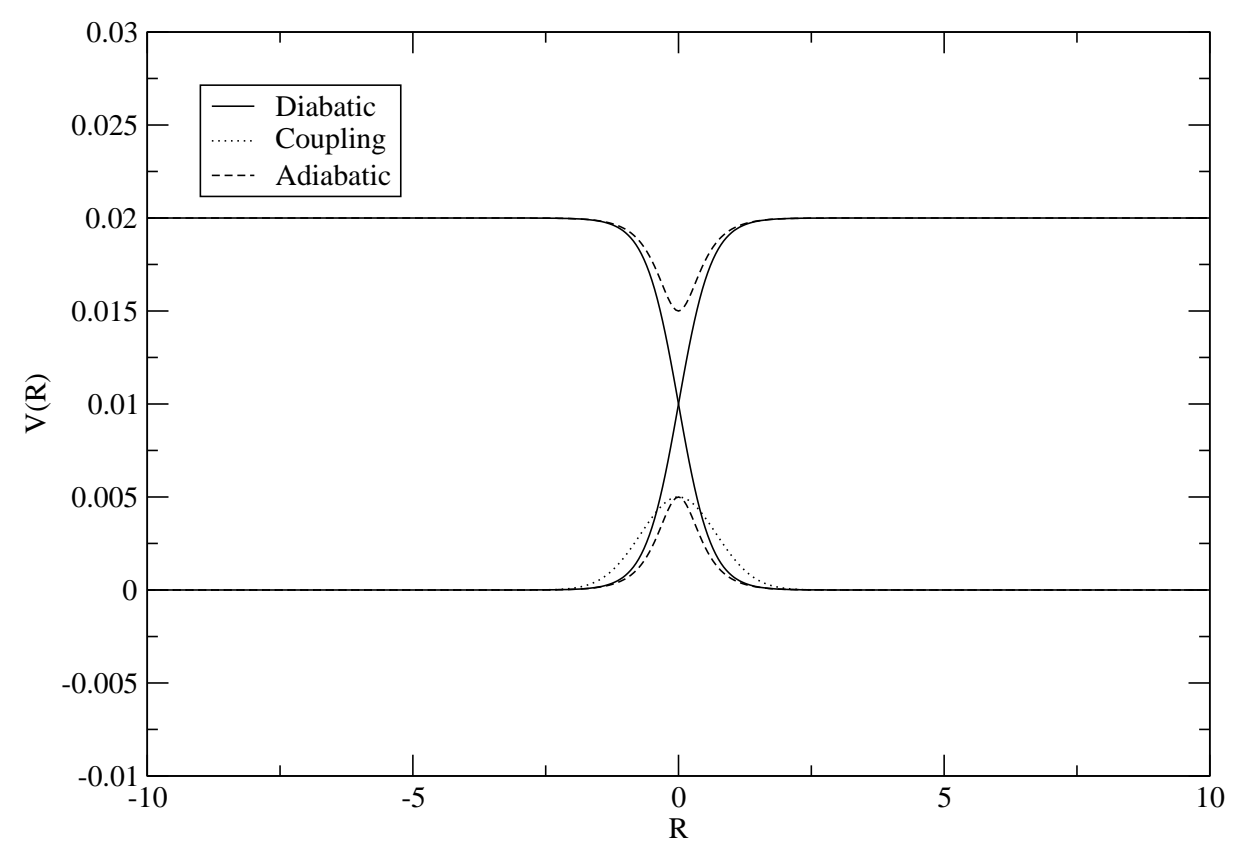

Figure 5.1: The Potential Curves in both Diabatic and Adiabatic Representation for Model 1

translational momentum, which can be expressed as the long time limit of the A-B correlation function of Eq. (3.8), where operator $\hat{A}$ is the projection operator for the initial state,

$$
\left|\Psi_{i}\right\rangle\left\langle\Psi_{i}\right|
$$

and operator $\hat{B}$ is

$$
\delta\left(P_{f}-\hat{P}\right)
$$




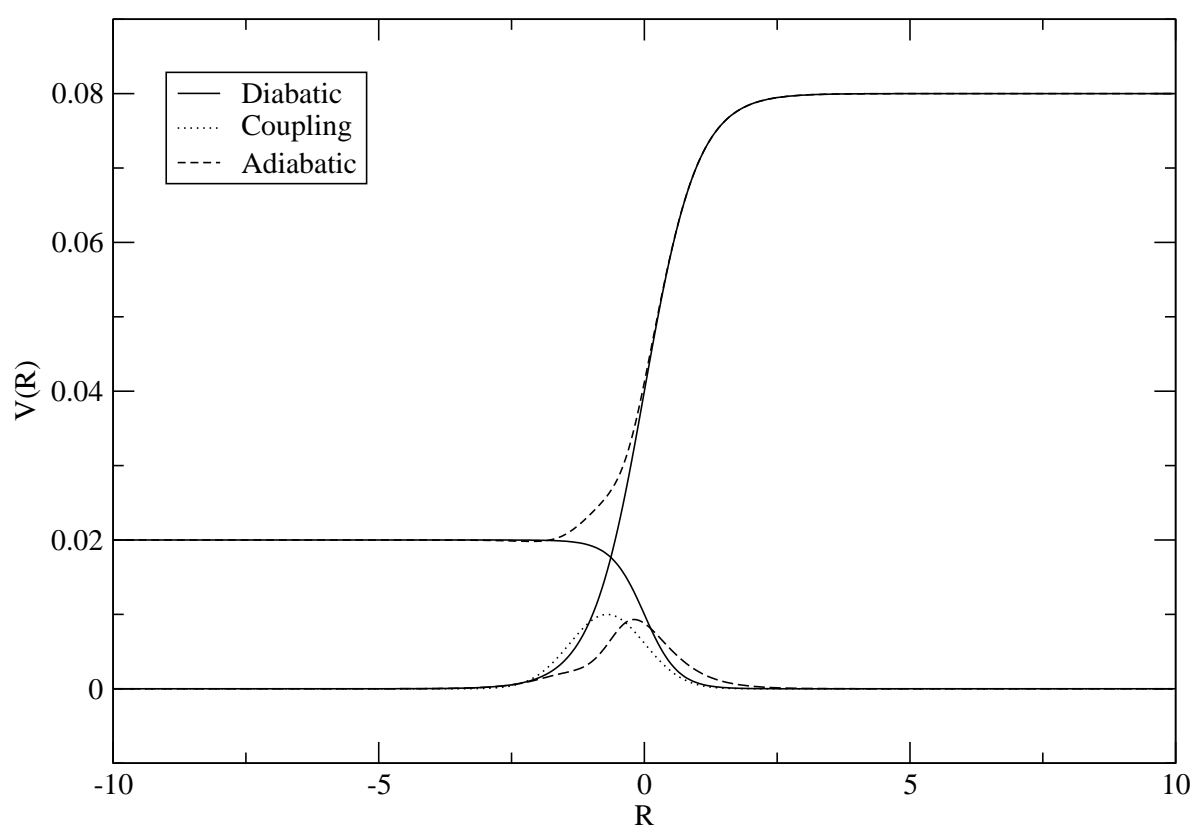

Figure 5.2: The Potential Curves in both Diabatic and Adiabatic Representation for Model 2

The correlation function is therefore,

$$
\begin{aligned}
C\left(P_{f} ; t\right) & =\lim _{t \rightarrow \infty} \operatorname{tr}\left[\left|\Psi_{i}\right\rangle\left\langle\Psi_{i}\right| e^{i \hat{H} t / \hbar} \delta\left(P_{f}-\hat{P} e^{-i \hat{H} t / \hbar}\right]\right. \\
& =\lim _{t \rightarrow \infty}\left\langle\Psi_{i}\left|e^{i \hat{H} t / \hbar} \delta\left(P_{f}-\hat{P}\right) e^{-i \hat{H} t / \hbar}\right| \Psi_{i}\right\rangle
\end{aligned}
$$

The initial state is chosen to be a translational coherent state (with a relatively well-defined value of the initial translational momentum) in electronic state 1 ,

$$
\Psi_{i}\left(x_{1}, x_{2}, P, R\right)=\left(\frac{\gamma}{\pi}\right)^{1 / 4} e^{-\frac{\gamma}{2}\left(R-R_{i}\right)^{2}} e^{i P_{i}\left(R-R_{i}\right)}\left(\frac{2}{\pi}\right)^{\frac{1}{2}} x_{1} e^{-\frac{1}{2}\left(x_{1}^{2}+x_{2}^{2}\right)}
$$

The initial position $R_{i}$ is chosen well to the left of the curve-crossing region $\left(R_{i}=-5\right)$, the initial momentum is positive, with trajectories headed toward the crossing region. 
For the LSC-IVR/classical Wigner approximation, it is necessary to calculate the

Wigner function for operator $\hat{A}$; this can be done analytically in this case, giving

$$
\begin{aligned}
A_{w} & =\left(\left|\Psi_{i}><\Psi_{i}\right|\right)_{w} \\
& =(2)^{3} e^{-\gamma\left(R-R_{i}\right)^{2}} e^{-\frac{1}{\gamma}\left(P-P_{i}\right)^{2}} e^{-\left(x_{1}^{2}+p_{1}^{2}+x_{2}^{2}+p_{2}^{2}\right)}\left(2 x_{1}^{2}+2 p_{1}^{2}-1\right)
\end{aligned}
$$

Operator $\hat{B}$ remains unchanged under the Wigner transformation.

For the FB-IVR implementation here, we Fourier expand the delta function of operator $\hat{B}$, so that Eq. (3.27) becomes

$$
C\left(P_{f} ; t\right)=(2 \pi \hbar)^{-1} \int_{-\infty}^{\infty} d \bar{R} e^{i \bar{R} \cdot P_{f} / \hbar}\left\langle\Psi_{i}\left|e^{i \hat{H} t} e^{-i \hat{P} \bar{R} / \hbar} e^{-i \hat{H} t}\right| \Psi_{i}\right\rangle
$$

and the FB-IVR is used to evaluate $\tilde{P}(\bar{R})$

$$
\begin{aligned}
\tilde{P}(\bar{R}) \equiv & \left\langle\Psi_{i}\left|e^{i \hat{H} t} e^{-i \hat{P} \bar{R} / \hbar} e^{-i \hat{H} t}\right| \Psi_{i}\right\rangle \\
= & (2 \pi \hbar)^{-F} \int d \mathbf{p}_{0} \int d \mathbf{q}_{0}\left\langle\Psi_{i} \mid \mathbf{p}_{0}^{\prime}, \mathbf{q}_{0}^{\prime}\right\rangle\left\langle\mathbf{p}_{0}, \mathbf{q}_{0} \mid \Psi_{i}\right\rangle \\
& C_{0}\left(\mathbf{q}_{0}, \mathbf{p}_{0}\right) e^{i S_{0}\left(\mathbf{q}_{0}, \mathbf{p}_{0}\right)} / \hbar
\end{aligned}
$$

The FB trajectory for Eq. (3.31) begins with initial conditions $\left(\mathbf{p}_{0}, \mathbf{q}_{0}\right)$, evolves to time $\mathrm{t}$ at which the translational coordinate undergoes a jump in the nuclear coordinate $R_{t} \rightarrow R_{t}+\bar{R}$ and then propagates back to time $0,\left(\mathbf{p}_{0}^{\prime}, \mathbf{q}_{0}^{\prime}\right)$ being the final values. Once $\tilde{P}(\bar{R})$ is thus calculated by this FB-IVR procedure, its Fourier 
transform gives $C\left(P_{f} ; t\right)$ by Eq. $(3.30)$

The Exact FB-IVR expression in this case is :

$$
\begin{aligned}
C\left(P_{f} ; t\right)= & (2 \pi \hbar)^{-F} \int d \mathbf{q}_{0} \int d \mathbf{p}_{0} \int d \mathbf{q}_{t}^{\prime} \mathcal{D}_{t}^{f}\left(\mathbf{q}_{0}, \mathbf{p}_{0} ; \gamma_{o}\right) \mathcal{D}_{-t}^{b}\left(\mathbf{q}_{t}^{\prime}, \mathbf{p}_{t}, \gamma_{o}^{\prime}\right) \delta\left(p_{t}^{r}-p_{f}\right) \\
& e^{\frac{i}{\hbar} S_{t}\left(q_{0}, p_{0}\right)} e^{\frac{i}{\hbar} S_{-t}\left(q_{t}^{\prime}, p_{t}\right)} e^{\frac{i}{\hbar} \mathbf{p}_{t} \cdot\left(\mathbf{q}_{t}^{\prime}-\mathbf{q}_{t}\right)}\left\langle\Psi_{i} \mid \mathbf{p}_{o}^{\prime}, \mathbf{q}_{o}^{\prime} ; \gamma_{o}^{\prime}\right\rangle\left\langle\mathbf{p}_{o}, \mathbf{q}_{o} ; \gamma_{o} \mid \Psi_{i}\right\rangle
\end{aligned}
$$

The trajectory has the same forward-backward structure as in the FB-IVR case. However, the position jump at time $t$ is over both the nuclear and the electronic co-ordinates.

\section{Discussion}

First, Figures 5.3 and 5.4 show the results of the FB-IVR and the EFB-IVR compared with exact quantum results for Model 1, at two incident translational energies, 0.03 and 0.1 hartree, respectively. These distributions show two peaks at positive momentum, corresponding to the two electronic states in which the system may emerge. The peaks have finite width because the initial translational wavefunction has a (small) spread in translational energy. The area under the peaks give the transition probabilities from the initial electronic state to the two possible final states, either for transmission $\left(P_{f}>0\right)$ or reflection $\left(P_{f}<0\right)$. The basic conclusion from Figures 5.3 and 5.4 is that the FB-IVR and EFB-IVR both describe the process semi-quantitatively, with the FB-IVR showing less numerical 
noise than the EFB-IVR (at least with our present calculations).

Figures 5.5 and 5.6 show the results of the FB-IVR calculations, compared to

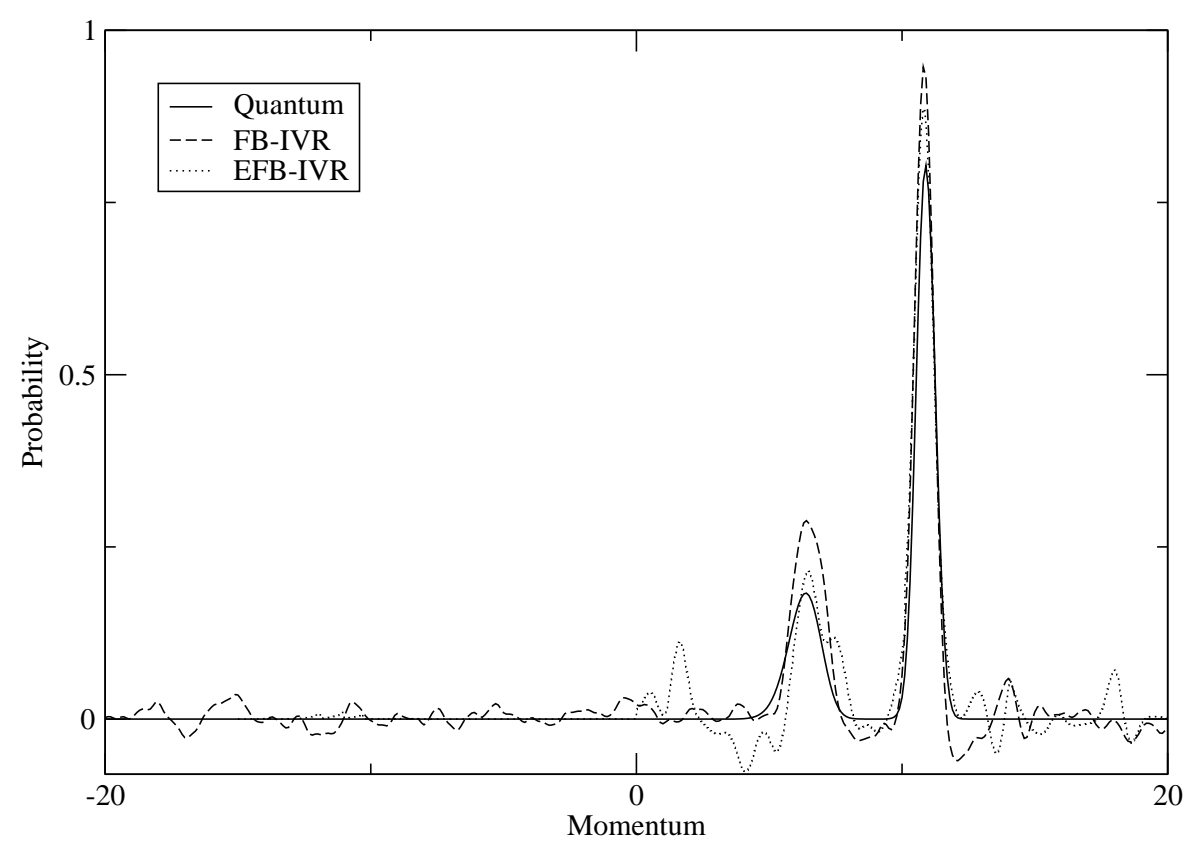

Figure 5.3: Comparison of results of FB-IVR, Exact FB-IVR and exact Quantum calculations for Model 1 with an initial energy of 0.03 Hartree

the exact quantum results, for Model 2 at the same two translational energies. For the lower energy case Figure 5.5 the excited electronic state is energetically forbidden (i.e., it is a closed channel) in transmission $\left(P_{f}>0\right)$, but both states are open for reflection $\left(P_{f}<0\right)$; the FB-IVR (and quantum result) correctly show one peak for positive momentum and two peaks for negative momentum. And again, the FB-IVR agrees well with the correct quantum result. At the higher energy case, shown in figure 5.6, both electronic states are open in transmission, and one sees two peaks for positive momentum and essentially no peaks correspond- 


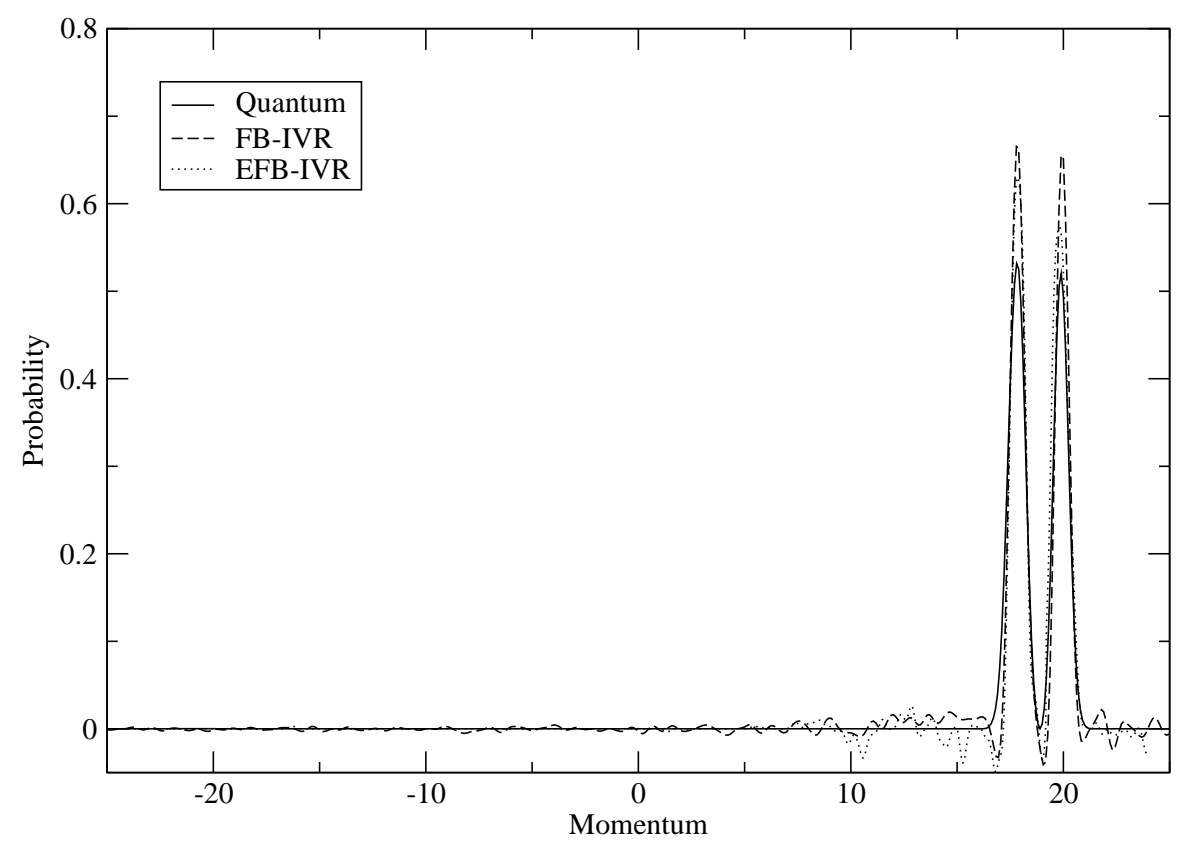

Figure 5.4: Comparison of results of FB-IVR, Exact FB-IVR and exact Quantum calculations for Model 1 with an initial energy of 0.1 Hartree

ing to reflected transitions. The Figures 5.7-5.10 show the results of the classical Wigner/LSC-IVR model, and also the standard Ehrenfest model, for models 1 and 2, for two initial nuclear translational energies, 0.03 and 0.1 hartree. One sees in Figures 5.7 and 5.8 (Model 1) that for each energy there is only a single peak in the translational energy distribution, clearly demonstrating that the translational motion has emerged on an average electronic potential curve, not one curve or the other. One also sees that the classical Wigner/LSC-IVR model is essentially the same as the standard Ehrenfest model, the primary difference being that since the initial conditions for the electronic variables have a distribution (the Wigner distribution) in values - while there is no distribution in the initial electronic variables in 


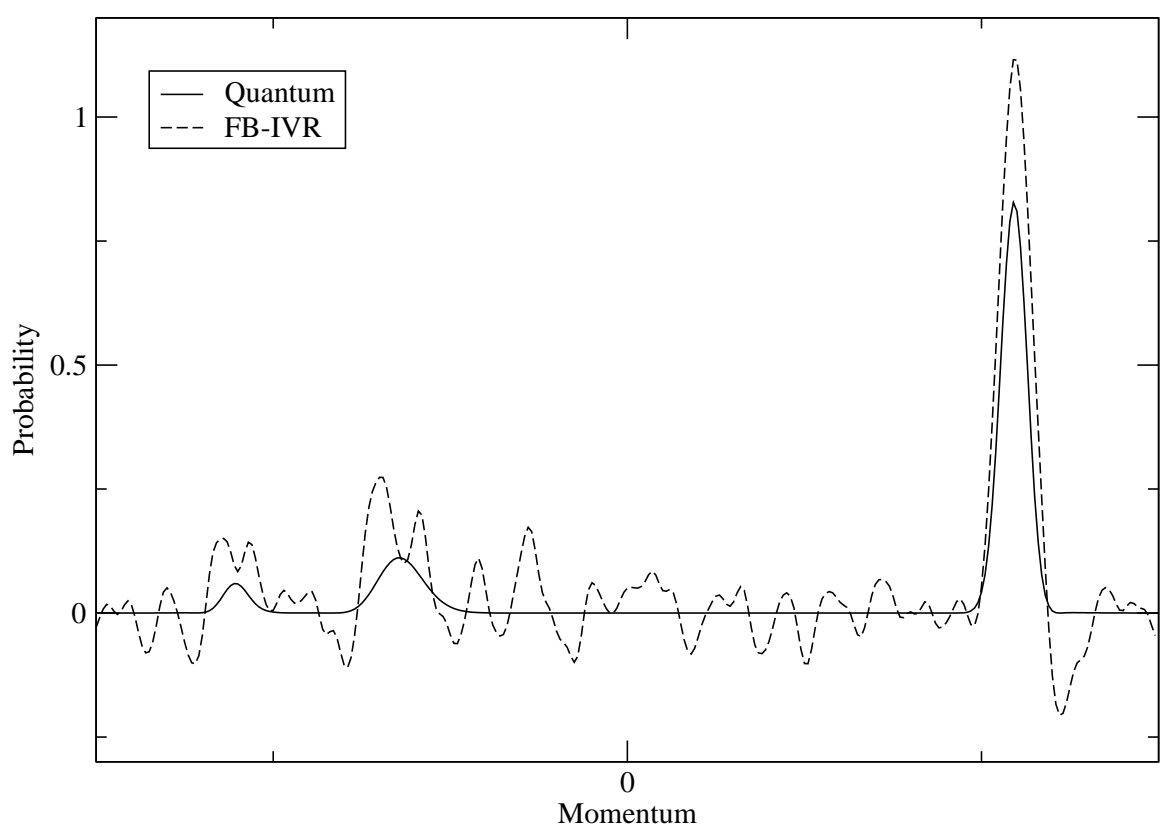

Figure 5.5: Comparison of results of FB-IVR, Exact FB-IVR and exact Quantum calculations for Model 2 with an initial energy of 0.03 Hartree

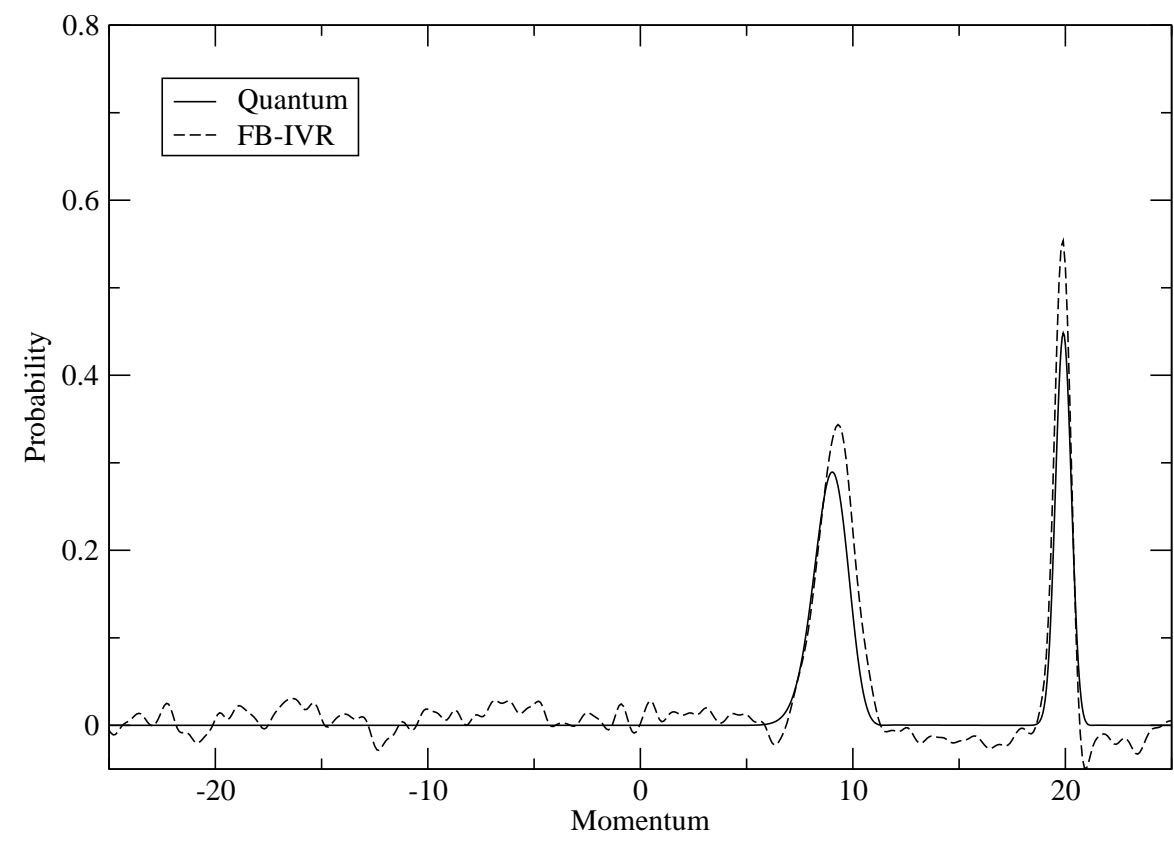

Figure 5.6: Comparison of results of FB-IVR, Exact FB-IVR and exact Quantum calculations for Model 2 with an initial energy of 0.1 Hartree 
the standard Ehrenfest model - there is a broadening in the very sharp peak given by the Ehrenfest model. (The Ehrenfest peak is in fact a delta function, so its width in Figures 5.7-5.10 is for visual purposes only.) Figures 5.9 and 5.10 shows similar behavior for Model 2; here the distribution given by the classical Wigner model is extremely broad for the lower energy case because there is a significant probability of reflection $\left(P_{f}<0\right)$ as well as transmission. The results in Figures 5.7-5.10 verify the unphysical behavior of the Ehrenfest model, and that the LSCIVR/classical Wigner model is essentially equivalent to it. These shortcomings are due to the fact that these models cannot describe quantum coherence/interference effects, which are necessary to properly quantize the electronic degrees of freedom.

Finally, Figures 5.11 and 5.12 show FB-IVR calculations for Model 1 at the two energies, comparing the results given by using the diabatic electronic representation (as did all calculations above) and the adiabatic electronic representation with Hamiltonian given by Eq. (2.20). The results should of course be the same, and within an extremely small numerical error they are.

The SC-IVR and LSC-IVR along with the MM-ST Hamiltonian have already been shown to be useful in the study of nonadiabatic dynamics. This section succesfully shows the effectiveness of the FB-IVR, which allows us to better evaluate quantities that involve double phase space integrals. We also further discuss the application of the LSC-IVR and compare it favorably to the Ehrenfest model. We also demonstrate the representation independent nature of our methods. It is im- 


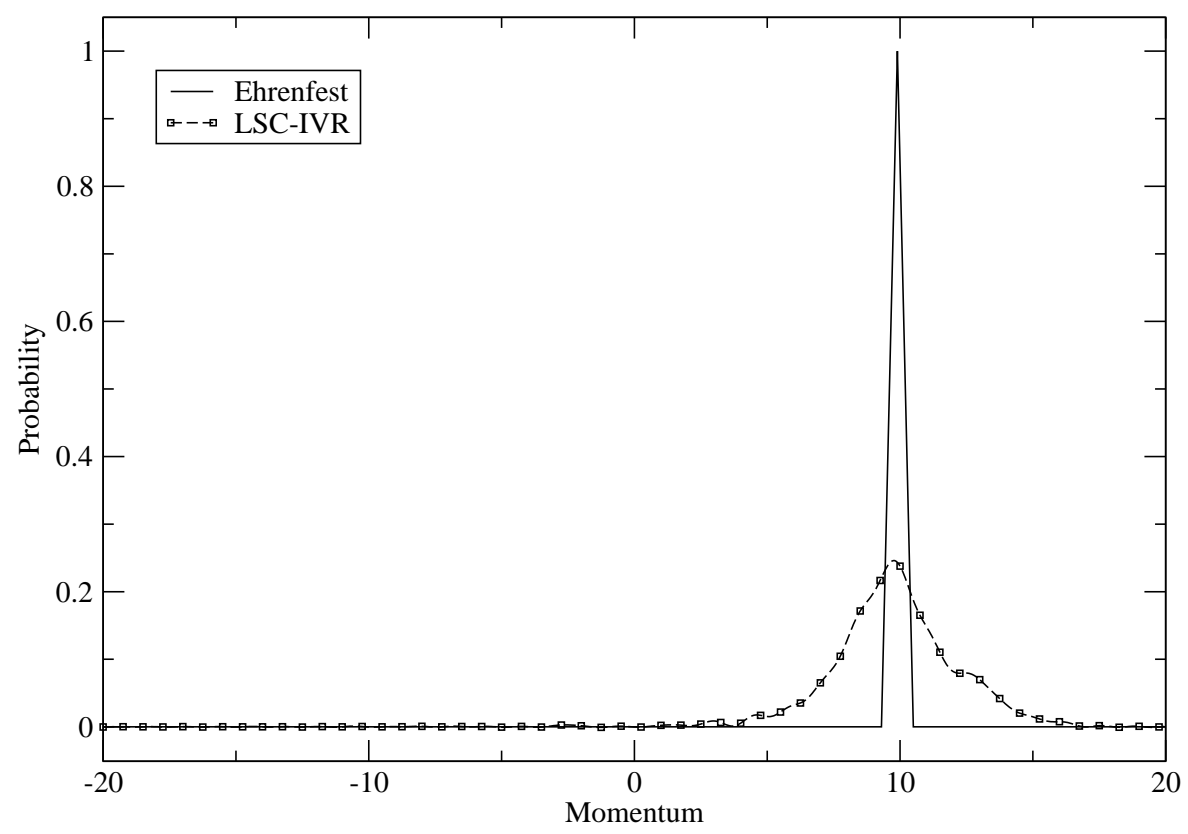

Figure 5.7: Linearised IVR and Ehrenfest results for Model 1. Initial Energy = 0.03 Hartree

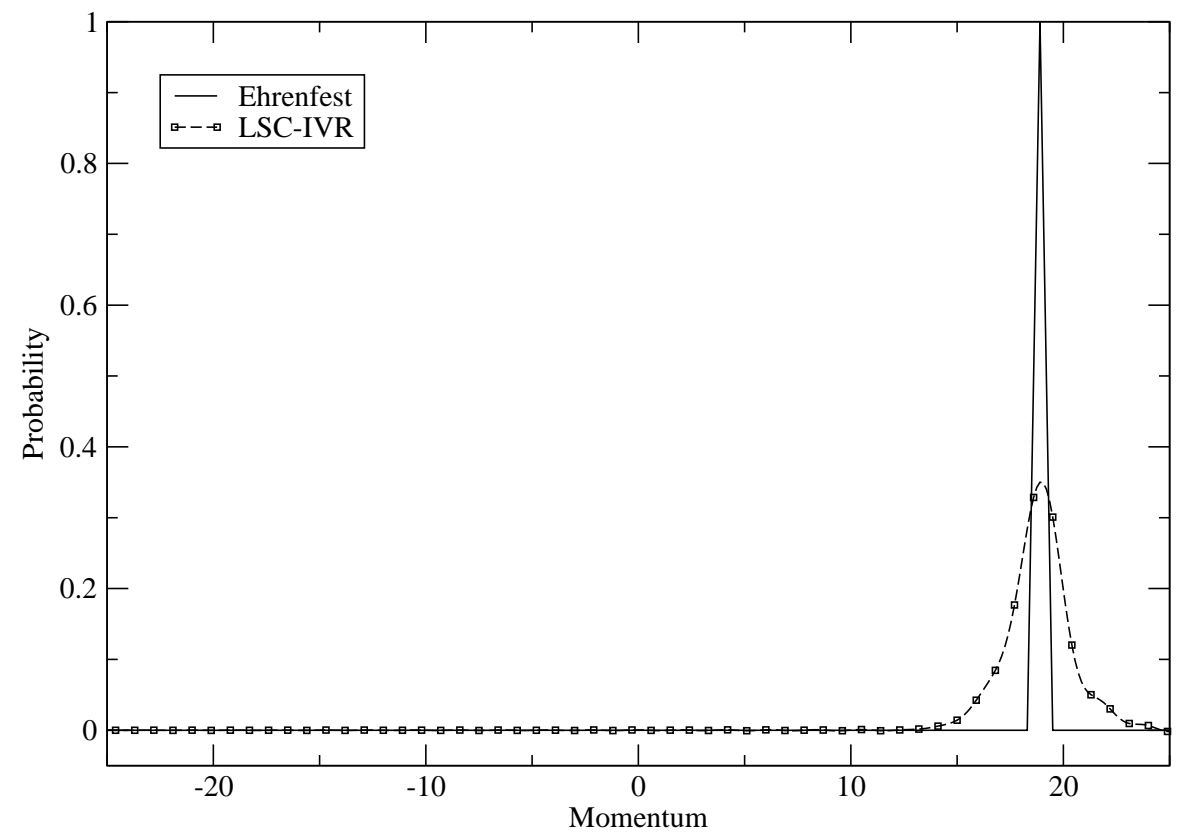

Figure 5.8: Linearised IVR and Ehrenfest results for Model 1. Initial Energy = 0.1 Hartree 


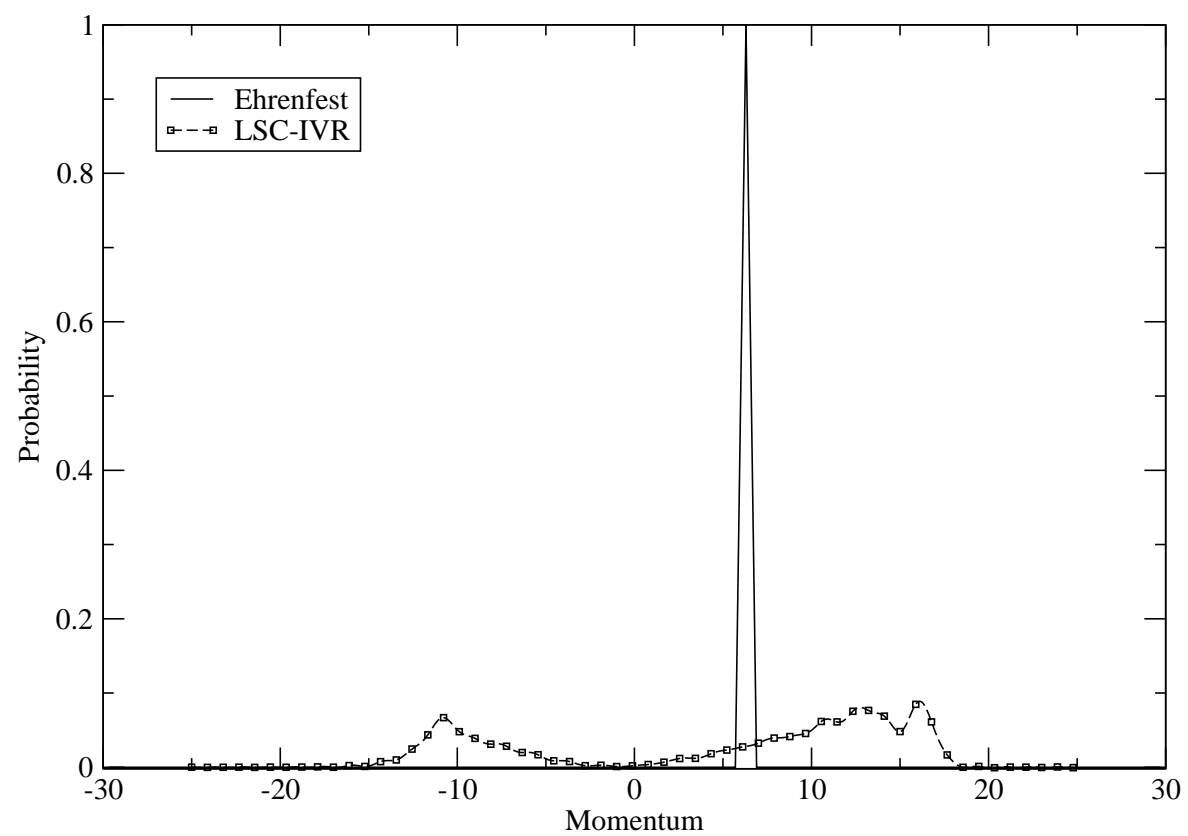

Figure 5.9: Linearised IVR and Ehrenfest results for Model 2. Initial Energy = 0.03 Hartree

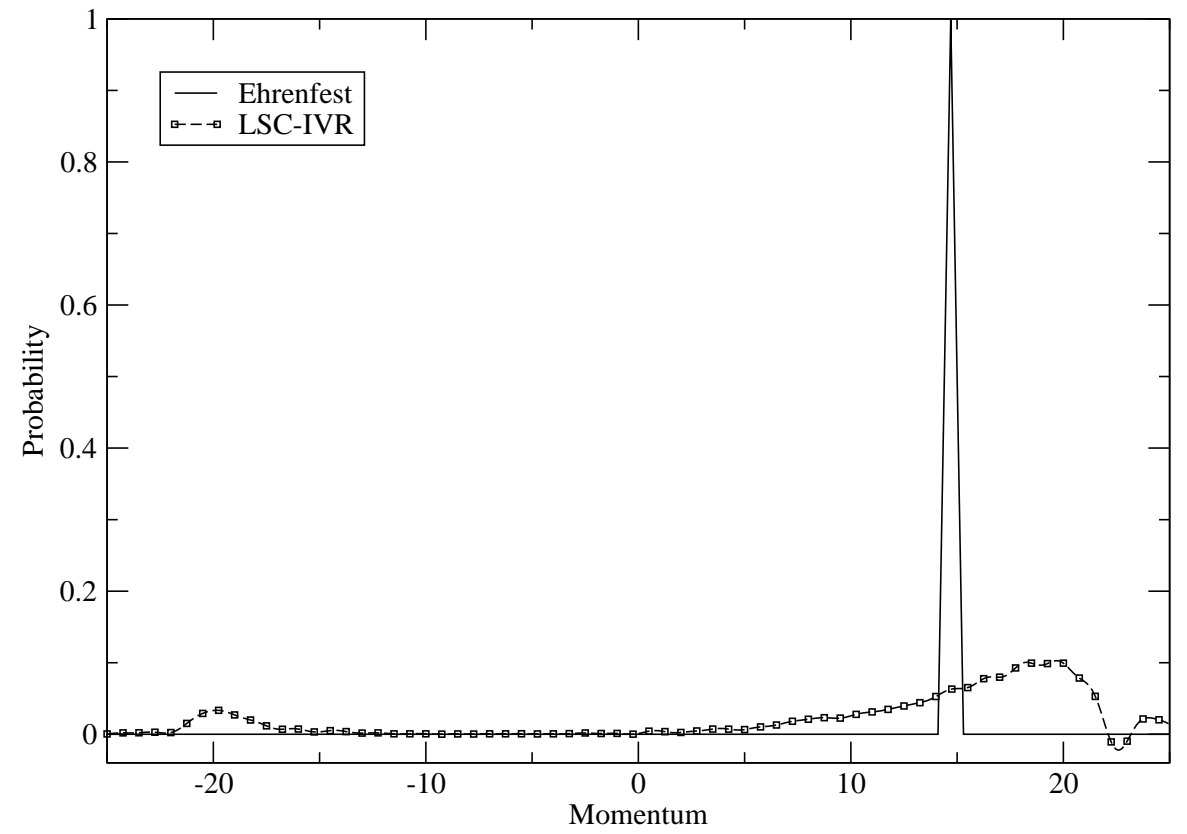

Figure 5.10: Linearised IVR and Ehrenfest results for Model 2. Initial Energy $=$ 0.1 Hartree 


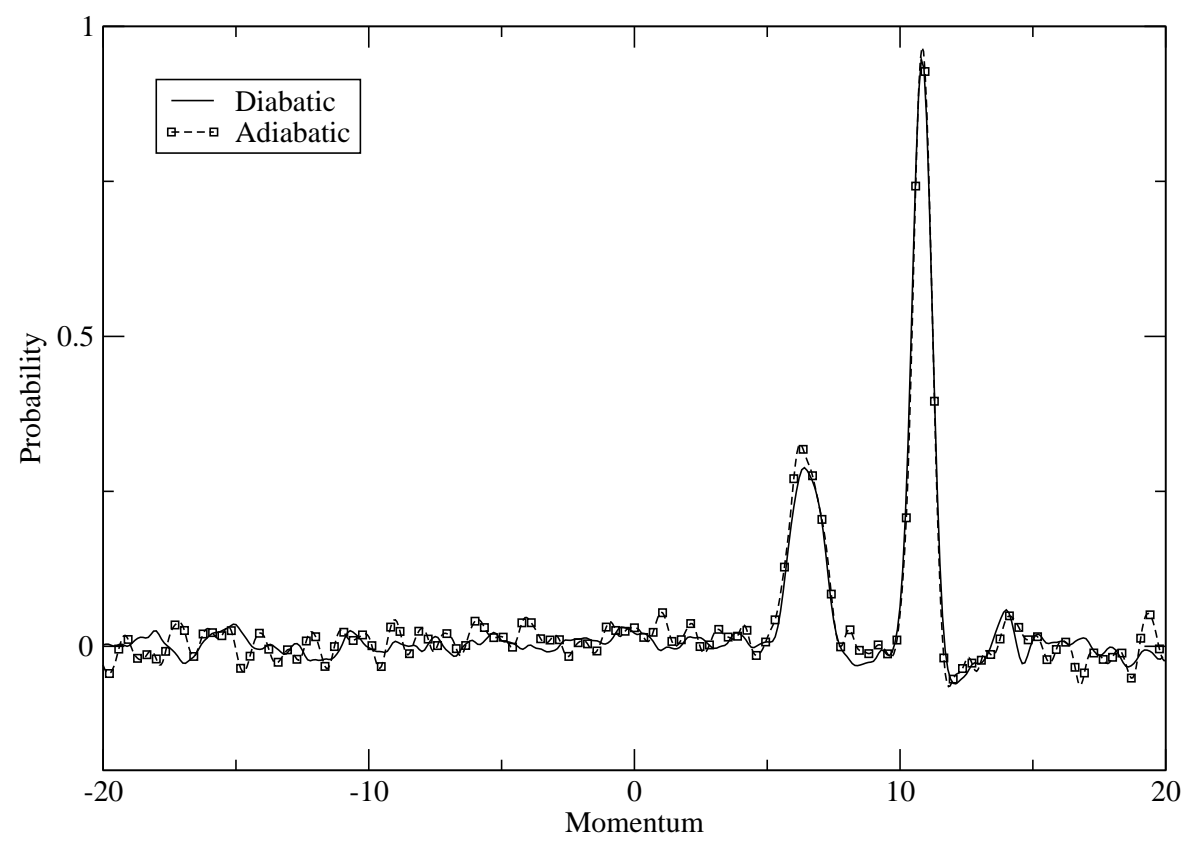

Figure 5.11: Comparison of the Adiabatic and Diabatic results for Model 1, with an initial energy 0.03 Hartree

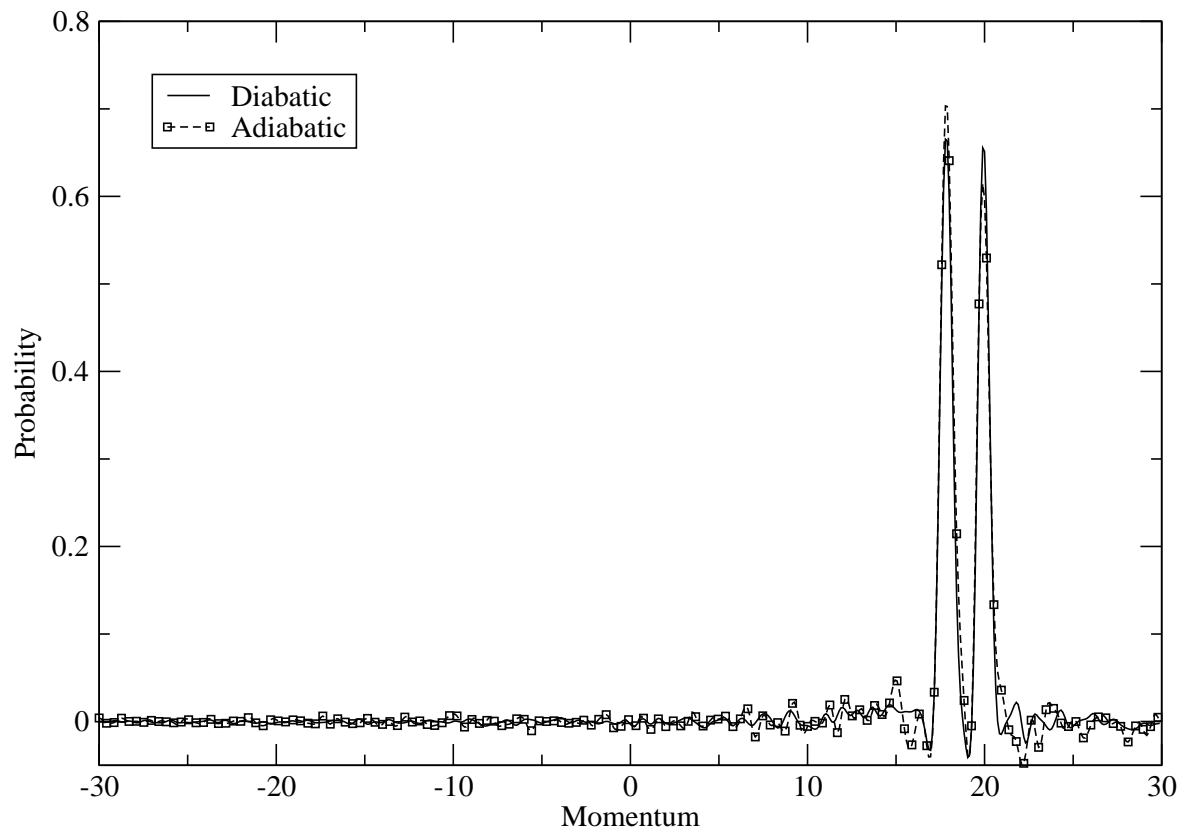

Figure 5.12: Comparison of the Adiabatic and Diabatic results for Model 1, with an initial energy 0.1 Hartree 
portant to note that both the FB-IVR and LSC-IVR are feasible for extension to multidimensional systems and in particular, the ability of FB-IVR to model nonadiabatic dynamics almost exactly shows that there need be no compromise on the incorporation of quantum effects in order to do so.

\section{Future Work}

We hope to use the techniques described above to simulate the behavior of more realistic systems. One system in particular we are looking at is the photodissociation of $\mathrm{Na}-\mathrm{FH}$ into $\mathrm{NaF}+\mathrm{H}$ or $\mathrm{NaH}+\mathrm{F}$. There is an avoided crossing in this particular system and with the model potential developed and used by Truhlar et al. ${ }^{70}$ We hope to show that the IVR can be used to predict the branching ratio. 


\section{Chapter 6}

\section{Tuning between the Quantum and}

\section{Classical Limits of SC-IVR -}

\section{Filinov Transformation}

\section{Introduction}

In dealing with complex systems, it becomes apparent in many cases that quantum effects are to a large extent important only for a few degrees of freedom. This makes it hard to justify dealing with the practical difficulties of treating large systems semiclassically in order to extract quantum effects from such a small part of the system. There are approaches that deal with this by doing a full quantum calculation for the relevant subsystem and treating the rest of the system classically. 
In such methods, the problem is putting these two parts together in a coherent way to obtain information about the complete system. For instance, if we think about this in terms of a system bath model, ${ }^{71}$ the bath has zero point energy effects that can affect the system through coupling between these modes. There is therefore, still virtue in doing a semiclassical simulation, but we would like to be able to be able to control exactly how much information we extract the quantum effects associated with the individual degree of freedom in the system.

Several exact and approximate forms of the IVR introduced to evaluate correlation functions were described earlier. Of these, the LSC-IVR is clearly the most easy to implement, while the EFB-IVR or the DHK-IVR are clearly the most difficult by virtue of there 'exact'-ness. The LSC-IVR is capable of capturing quantum effects in a limited way; coherence and interference effects remain entirely beyond its reach as was shown, for instance, in the chapter on nonadiabatic dynamics. This then, would be our tool of choice when working with the bath modes of a system-bath model, for example. While choosing the tools for the 'system' part, we would go with a more exact formalism such as the EFB-IVR. The ideal approach would allow us to do both in one calculation.

In this chapter, we describe exactly such a method. The Modified Filinov transformation is applied to a DHK-IVR expression for a correlation function. We show that the filinov parameter acts as a tuning factor such that in one limit we obtain an LSC-IVR type of formalism and in the other limit, we obtain an EFB-IVR ex- 
pression. Using this Tuning-IVR (TIVR), we can now do exactly what we talked about in the previous paragraph. The filinov factor appears as a diagonal matrix whose dimensions are equal to the number of degrees of freedom in the system and by tuning each element of the diagonal we can determine which limit we would like to look at for the corresponding degree of freedom (or if we want to settle for some intermediate level too.)

This idea is based on previous work done to derive a Generalized FB-IVR (GFBIVR $)^{72}$ and is an extension of that derivation. The GFB-IVR tuned between the FB-IVR and the DHK-IVR. In the next section we describe our approach and the derivation of this TIVR and show its application with a 1D model and a systembath model.

\section{Theory}

\section{II.1 Modified Filinov Transformation}

The modified Filinov method was originally developed to help carry out Monte Carlo integration of Feynman path integrals. ${ }^{73}$ Here we briefly outline the details of the method. Consider a one-dimensional integral of the form

$K=\int_{-\infty}^{\infty} d x e^{i S(x)}$ 
We can insert unity in the form of a normalized gaussian integral

$$
1=\int_{-\infty}^{\infty} d x_{0} \sqrt{\frac{B}{2 \pi}} e^{-\frac{1}{2} B\left(x-x_{0}\right)^{2}}
$$

The order of integration is interchanged and we obtain

$K=\int_{-\infty}^{\infty} d x_{0} \sqrt{\frac{B}{2 \pi}} \int_{-\infty}^{\infty} d x e^{i S(x)-\frac{1}{2} B\left(x-x_{0}\right)^{2}}$

The Gaussian factor makes the values of $x$ near $x_{0}$ contribute the most to the value of the integral. This allows us to expand the function $S(x)$ about $x_{0}$ to 2 nd order. $S(x) \approx S\left(x_{0}\right)+S^{\prime}\left(x_{0}\right)(x-x 0)+\frac{1}{2} S^{\prime \prime}\left(x_{0}\right) *\left(x-x_{0}\right)^{2}$

and the integral over $x$ can now be carried out

$$
\begin{aligned}
K & \approx K(B) \\
& \equiv \int_{-\infty}^{\infty} d x_{0} e^{i S\left(x_{0}\right)\left[1-\frac{i S^{\prime \prime}\left(x_{0}\right)}{B}\right]^{-\frac{1}{2}}} e^{-\frac{\frac{1}{2} S^{\prime}\left(x_{0}\right)^{2}}{\left[B-i S^{\prime \prime}\left(x_{0}\right)\right]}}
\end{aligned}
$$

In the original Filinov method B was taken to be real. The modified Filinov method uses the fact that Eq. (2.1) is valid if B is complex and approximately true if B is a function of $x_{0}$. So we now allow $\mathrm{B}$ to be such a function and further make a specific choice

$B\left(x_{0}\right)=i S^{\prime \prime}\left(x_{0}\right)+c^{-1}$

where $\mathrm{c}$ is some constant. The equation then reduces to

$$
\begin{aligned}
K & \approx K(c) \\
& \equiv \int_{-\infty}^{\infty} d x e^{i S(x)}\left[1+i c S^{\prime \prime}(x)\right]^{\frac{1}{2}} e^{-\frac{1}{2} c\left[S^{\prime}(x)\right]^{2}}
\end{aligned}
$$

where the integration variable has been renamed $x$ The Monte Carlo integration is then performed with the unnormalized sampling 
function that is in the integrand. We note that in the limit of $c \rightarrow \infty$, the transformation is equivalent to evaluating the stationary phase approximation to the integral.

\section{II.2 GFB-IVR}

The Generalized FB-IVR utilizes the modified Filinov method described in the previous section to a somewhat different end. It has also been noted that in the limit of $c \rightarrow \infty$ the integrand in Eq. (2.4) approaches the stationary phase limit. We apply this idea to the double HK-IVR for a generic correlation function that has already been described in a previous chapter. We look at the expression as being of the form

$I=\int d \mathbf{z} g(\mathbf{z}) e^{i f(\mathbf{z})}$

where we define the vector $\mathbf{z}$ as a 4 N-dimensional vector of form $\mathbf{z}=\left(\mathbf{q}_{\mathbf{t}}^{\prime}, \mathbf{p}_{\mathbf{t}}^{\prime}, \mathbf{q}_{\mathbf{0}}, \mathbf{p}_{\mathbf{0}}\right)$ and

$g(\mathbf{z})=C_{t}\left(\mathbf{p}_{\mathbf{0}}, \mathbf{q}_{\mathbf{0}}\right) C_{-t}\left(\mathbf{p}_{\mathbf{t}}^{\prime}, \mathbf{q}_{\mathbf{t}}^{\prime}\right) \Pi_{j=1}^{N}\left(\frac{\gamma_{j}}{\pi}\right)^{\frac{1}{2}}$

The phase function $f(\mathbf{z})$ involves the action and the exponents of various coherent state(s) present in the expression. We will not reproduce the GFB-IVR derivation here since it is described in detail elsewhere. ${ }^{72}$ 


\section{II.3 A Tuning IVR (TIVR)}

The procedure we follow for this derivation closely follows the derivation of the GFB-IVR. We apply the modified filinov method to the double HK-IVR; it deviates from the previous only in that we make sure to differentiate between the width of the coherent state and time zero and at time $t$ and later use this to our advantage. This gives us,

$$
\begin{aligned}
C_{A B}(t)= & \left(\frac{1}{2 \pi \hbar}\right)^{F} \int d \mathbf{p}_{\mathbf{0}} \int d \mathbf{q}_{\mathbf{0}} \int d \mathbf{p}_{\mathbf{t}}^{\prime} \int d \mathbf{q}_{\mathbf{t}}^{\prime}\left\langle\mathbf{p}_{\mathbf{0}}, \mathbf{q}_{\mathbf{0}} \mid \psi_{i}\right\rangle\left\langle\psi_{i} \mid \mathbf{p}_{\mathbf{0}}^{\prime}, \mathbf{q}_{\mathbf{0}}^{\prime}\right\rangle \\
& \left\langle\mathbf{p}_{\mathbf{t}}^{\prime}, \mathbf{q}_{\mathbf{t}}^{\prime} \mid \mathbf{p}_{\mathbf{t}}, \mathbf{q}_{\mathbf{t}}\right\rangle F\left(\mathbf{p}_{\mathbf{t}}^{\prime}, \mathbf{q}_{\mathbf{t}}^{\prime}, \mathbf{p}_{\mathbf{t}}, \mathbf{q}_{\mathbf{t}}\right) e^{i\left(S_{t}+S_{-t}\right)} C_{F} \\
& e^{-\frac{1}{2} \mathbf{c}_{\mathbf{q}}\left(\mathbf{q}_{\mathbf{t}}^{\prime}-\mathbf{q}_{\mathbf{t}}\right)^{2}} e^{-\frac{1}{2} \mathbf{c}_{\mathbf{p}}\left(\mathbf{p}_{\mathbf{t}}^{\prime}-\mathbf{p}_{\mathbf{t}}\right)^{2}}
\end{aligned}
$$

We define a few of the variables and functions that appear in our equation here. $F\left(\mathbf{p}_{\mathbf{t}}^{\prime}, \mathbf{q}_{\mathbf{t}}^{\prime}, \mathbf{p}_{\mathbf{t}}, \mathbf{q}_{\mathbf{t}}\right)$ is defined as

$$
\left\langle\mathbf{p}_{\mathbf{t}}^{\prime}, \mathbf{q}_{\mathbf{t}}^{\prime}|\hat{B}| \mathbf{p}_{\mathbf{t}} \mathbf{q}_{\mathbf{t}}\right\rangle=\left\langle\mathbf{p}_{\mathbf{t}}^{\prime}, \mathbf{q}_{\mathbf{t}}^{\prime} \mid \mathbf{p}_{\mathbf{t}}, \mathbf{q}_{\mathbf{t}}\right\rangle F\left(\mathbf{p}_{\mathbf{t}}^{\prime}, \mathbf{q}_{\mathbf{t}}^{\prime}, \mathbf{p}_{\mathbf{t}}, \mathbf{q}_{\mathbf{t}}\right)
$$

and the prefactor is defined by

$$
C_{F}=\operatorname{det}\left|C_{t}\left(p_{0}, q_{0}\right) C_{-t}\left(p_{t}^{\prime}, q_{t}^{\prime}\right) \frac{\left(\mathbf{K}+\mathbf{i} \mathbf{J}^{\mathbf{T}} \mathbf{c}\right)}{\mathbf{K}}\right|^{\frac{1}{2}}
$$

Before taking either of the Filinov parameter to its stationary phase limit, we first go make the transformation to the EFB-IVR limit by taking $\gamma_{t} \rightarrow \infty$; The 
exactness of our equation thus far does not change. We now have,

$$
\begin{aligned}
C_{A B}(t)= & \left(\frac{1}{2 \pi \hbar}\right)^{F / 2} \int d \mathbf{p}_{\mathbf{0}} \int d \mathbf{q}_{\mathbf{0}} \int d \mathbf{p}_{\mathbf{t}}^{\prime}\left\langle\mathbf{p}_{\mathbf{0}}, \mathbf{q}_{\mathbf{0}} \mid \psi_{i}\right\rangle\left\langle\psi_{i} \mid \mathbf{p}_{\mathbf{0}}^{\prime}, \mathbf{q}_{\mathbf{0}}^{\prime}\right\rangle F\left(\mathbf{p}_{\mathbf{t}}^{\prime}, \mathbf{p}_{\mathbf{t}}, \mathbf{q}_{\mathbf{t}}\right) \\
& e^{i\left(S_{t}+S_{-t}\right)} \bar{C} e^{-\frac{1}{2} \mathbf{c}_{\mathbf{p}}\left(\mathbf{p}_{\mathbf{t}}^{\prime}-\mathbf{p}_{\mathbf{t}}\right)^{2}}
\end{aligned}
$$

(We further note that since we take the limit of large $\gamma_{t}$ we can assume the matrix to be a constant and thus not worry about the order of multiplication in the multidimensional case)

$$
\begin{aligned}
\pi \bar{C} & =\operatorname{det} \mid\left(\frac { 1 } { 4 \pi \gamma _ { 0 } } \left[\left(-\mathbf{M}_{q p}^{b} \gamma_{0}-i \mathbf{M}_{p p}^{b}\right)\left(i \mathbf{M}_{q q}^{f}+\gamma_{0} \mathbf{M}_{q p}^{f}\right)\right.\right. \\
& +\left(-\mathbf{M}_{q p}^{b} \gamma_{0}-i \mathbf{M}_{p p}^{b}\right)\left(i \gamma_{0} \mathbf{M}_{p p}^{f}-\mathbf{M}_{p q}^{f}\right) \mathbf{c}_{\mathbf{p}} \\
& \left.+\left(-\mathbf{M}_{q q}^{b} \gamma_{0}-i \mathbf{M}_{p q}^{b}\right) \mathbf{c}_{\mathbf{p}}\left(-\mathbf{M}_{q q}^{f}+i \gamma_{0} \mathbf{M}_{q p}^{f}\right)\right]\left.\right|^{\frac{1}{2}}
\end{aligned}
$$

We now examine both limits of the filinov parameter in 1D for simplicity.

First, the limit $\mathbf{c}_{\mathbf{p}} \rightarrow 0$. The momentum jump at time $t$ can have any values effectively since it is described by a gaussian of infinite width. The expression for the correlation function reduces to the EFB-IVR expression :

$$
\begin{aligned}
C_{A B}(t)= & \left(\frac{1}{2 \pi \hbar}\right)^{F / 2} \int d \mathbf{p}_{\mathbf{0}} \int d \mathbf{q}_{\mathbf{0}} \int d \mathbf{p}_{\mathbf{t}}^{\prime}\left\langle\mathbf{p}_{\mathbf{0}}, \mathbf{q}_{\mathbf{0}} \mid \psi_{i}\right\rangle\left\langle\psi_{i} \mid \mathbf{p}_{\mathbf{0}}^{\prime}, \mathbf{q}_{\mathbf{0}}^{\prime}\right\rangle \\
& F\left(\mathbf{q}_{\mathbf{t}}\right) e^{i\left(S_{t}+S_{-t}\right)} D_{f}
\end{aligned}
$$

where we have also made the further assumption (only in the interests of simplicity) that operator B is a function of only position space operator. The prefactor $D_{f}$ is now

$$
D_{f}=\left(\frac{1}{\sqrt{2 \pi}}\left(-i \gamma_{0} \mathbf{M}_{q p}^{b}+\mathbf{M}_{p p}^{b}\right)\right)^{\frac{1}{2}}\left(\frac{1}{\sqrt{2 \pi}}\left(\frac{1}{\gamma_{0}} \mathbf{M}_{q q}^{f}-i \mathbf{M}_{q p}^{f}\right)\right)^{\frac{1}{2}}
$$


Now lets look at the other limit (the stationary phase limit as defined before) where $\mathbf{c}_{\mathbf{p}} \rightarrow \infty$

$$
\begin{aligned}
& C_{A B}(t)=\left(\frac{1}{2 \pi \hbar}\right)^{F / 2} \int d \mathbf{p}_{\mathbf{0}} \int d \mathbf{q}_{\mathbf{0}} \quad\left\langle\mathbf{p}_{\mathbf{0}}, \mathbf{q}_{\mathbf{0}} \mid \psi_{i}\right\rangle\left\langle\psi_{i} \mid \mathbf{p}_{\mathbf{0}}^{\prime}, \mathbf{q}_{\mathbf{0}}^{\prime}\right\rangle \\
& F\left(\mathbf{q}_{\mathbf{t}}\right) e^{i\left(S_{t}+S_{-t}\right)} C_{H K}^{f u l l}
\end{aligned}
$$

In this limit, we note that the Filinov parametrized gaussian is reduced to a delta function

$$
\lim _{\mathbf{c}_{\mathbf{p}} \rightarrow \infty} e^{-\frac{1}{2} \mathbf{c}_{\mathbf{p}}\left(\mathbf{p}_{\mathbf{t}}^{\prime}-\mathbf{p}_{\mathbf{t}}\right)^{2}}=\delta\left(\mathbf{p}_{\mathbf{t}}^{\prime}-\mathbf{p}_{\mathbf{t}}\right)\left(\frac{2 \pi}{\mathbf{c}_{\mathbf{p}}}\right)^{\frac{1}{2}}
$$

The prefactor here is called $C_{H K}^{f u l l}$ by which we mean that the monodromy matrices that we evaluate are of the form $\mathbf{M}_{p p}^{f u l l}=\frac{\partial \mathbf{p}_{0}^{\prime}}{\partial \mathbf{p}_{\mathbf{0}}}$ and so on. Further we note that the coherent state limit set the position jump to zero and when the gaussian in momentum jump is reduced to a delta function, we no longer have a position jump either. Physically, we see that the trajectory that goes forward is identical with the backward trajectory; the action exactly cancels and the monodromy matrices at time $t$ are identical with their values at time zero, so the prefactor is just 1 . This further reduces our expression for the correlation function down to the form

$$
C_{A B}(t)=\left(\frac{1}{2 \pi \hbar}\right)^{F / 2} \int d \mathbf{p}_{\mathbf{0}} \int d \mathbf{q}_{\mathbf{0}}\left|\left\langle\mathbf{p}_{\mathbf{0}}, \mathbf{q}_{\mathbf{0}} \mid \psi_{i}\right\rangle\right|^{2} F\left(\mathbf{q}_{\mathbf{t}}\right)
$$

This expression looks a lot like the LSC-IVR formulation, except instead of the sampling being done with a Wigner distribution of initial states we have a Husimi distribution. 


\section{Applications}

In order to best showcase the properties of this TIVR we choose to first study a simple 1D system where there is an clear distinction between its behavior in a classical simulation and a quantum one. Once the effectiveness of this method in going between the two limits has been established, we move on to looking at a system-bath model which can demonstrate the ability of this system to extend itself to more degrees of freedom.

\section{III.1 Anharmonic Oscillator}

We look at the way the average position changes in time for an anharmonic oscillator. Initially, the 'exact' or 'quantum' time evolution follows the classical time evolution. However, the wavepacket begins to splits and dephase due to the anharmonicity and in time there is rephasing of the wavepacket to varying degrees. The dephasing and rephasing of the wavepacket can be seen by studying the change in average position with time. The correlation function for this property is based on the Eq. (3.8) where the operator A is a projection operator,

$$
\hat{A}=\left|\psi_{i}\right\rangle\left\langle\psi_{i}\right|
$$

Operator B is the position operator $\hat{x}$.

The correlation function is, thus of the form

$$
C_{A B}(t)=\left\langle\psi_{i}\left|e^{i \hat{H} t / \hbar} \hat{x} e^{-i \hat{H} t / \hbar}\right| \psi_{i}\right\rangle
$$


The Hamiltonian takes the form

$$
\hat{H}=\frac{\mathbf{p}^{2}}{2 m}+\frac{1}{2} \omega^{2} \mathbf{x}^{2}+a \mathbf{x}^{3}+b \mathbf{x}^{4}
$$

where we choose

$a=-0.1$ and $b=0.1$ and $w=\sqrt{(2)}$

The reference state is chosen to be the shifted ground state of the corresponding harmonic oscillator.

$$
\left\langle x \mid \psi_{i}\right\rangle=\left(\frac{\gamma}{\pi}\right) e^{-\frac{\gamma}{2}(x-1)^{2}}
$$

The width of the coherent state is chosen to be such that $\gamma=\omega$.

\section{III.2 System-Bath Model}

We now couple this anharmonic oscillator to a 30-dimensional bath of harmonic oscillators in an effort to see the beginnings of dissipation effects. ${ }^{74}$ The bath frequencies and coupling constants are distributed according to an Ohmic spectral density. The discretization of the spectral density function is carried out by a procedure described previously. ${ }^{75}$

The bath is characterized by its spectral density

$$
J(\omega)=\frac{\pi}{2} \sum_{j} \frac{\kappa_{j}^{2}}{\omega_{j}} \delta
$$

which is chosen to be in Ohmic form

$$
J(\omega)=\eta \omega e^{-\omega / \omega_{c}}
$$


The cut-off frequency is chosen to be $\omega_{c}=\omega$ and the maximum bath frequency is chosen to be $w_{\max }=2 \omega_{c}$. We also do the simulation in the weak coupling region where $\eta=0.1$

\section{III.3 Tracking the Prefactor}

It is worth discussing briefly what is involved in tracking the prefactor in Eq. (2.10). There is a difference between this and the other prefactors in the sense that we can no longer separate the monodromy matrices for the forward and backward trajectories. For instance, in Eq. (2.11) the two parts of the prefactor (the forward and the backward) can be calculated separately and tracked separately and then put together for the overall prefactor. In this case the prefactor is not really separable in that convenient way, so the procedure to evaluate this is a little more complicated. In the interests of storing as little information as possible, the method we use is as follows

First, the forward trajectory is evaluated in short time steps of $d t$ with the trajectory variables all stored at the end of the time evolution so that it can be used as the starting point for the next forward time evolution by $d t$. Next, the backward trajectory is started at time $n . d t$ and evolved back in time to zero. By combining the Monodromy matrices for the backward trajectory with the forward stored matrices, we can calculate and track the prefactor. 
In terms of the Maslov index, one further computational detail is to be noted. In the limit $\mathbf{c}_{\mathbf{p}} \rightarrow 0$, we know that the prefactor must reduce to the EFB-IVR limit. However, since we count the branch cuts for the non-separable form we will not necessarily end up with an identical solution. In order to ensure that the limits work properly, one trick we can use is to simply track the components of the EFBIVR prefactor and use the Maslov index obtained in this way with the Filinov parametrized prefactor. While this is exact in the one limit and irrelevant in the other limit, there is some ambiguity as to the validity of this procedure when $\mathbf{c}_{\mathbf{p}}$ lies somewhere in between. This is something we hope to further investigate.

\section{Discussion}

The results obtained thus far are promising, but we note that this work is ongoing and therefore none of the figures are in a final form.

For the anharmonic oscillator, we show the results of an exact (DVR) calculation in Figure 6.1 and compare that with the DHK-IVR and the EFB-IVR. It is clear that the EFB-IVR does not seem to be able to capture the quantum recurrences in the average position with time; however, there appears to be a loss of amplitude somewhat faster than expected and therefore smaller recurrences. To check the accuracy of our EFB-IVR we compare it with the DHK-IVR result where $\gamma_{t}$ is set to a large number. We definitely start to see some decrease in the amplitude of 


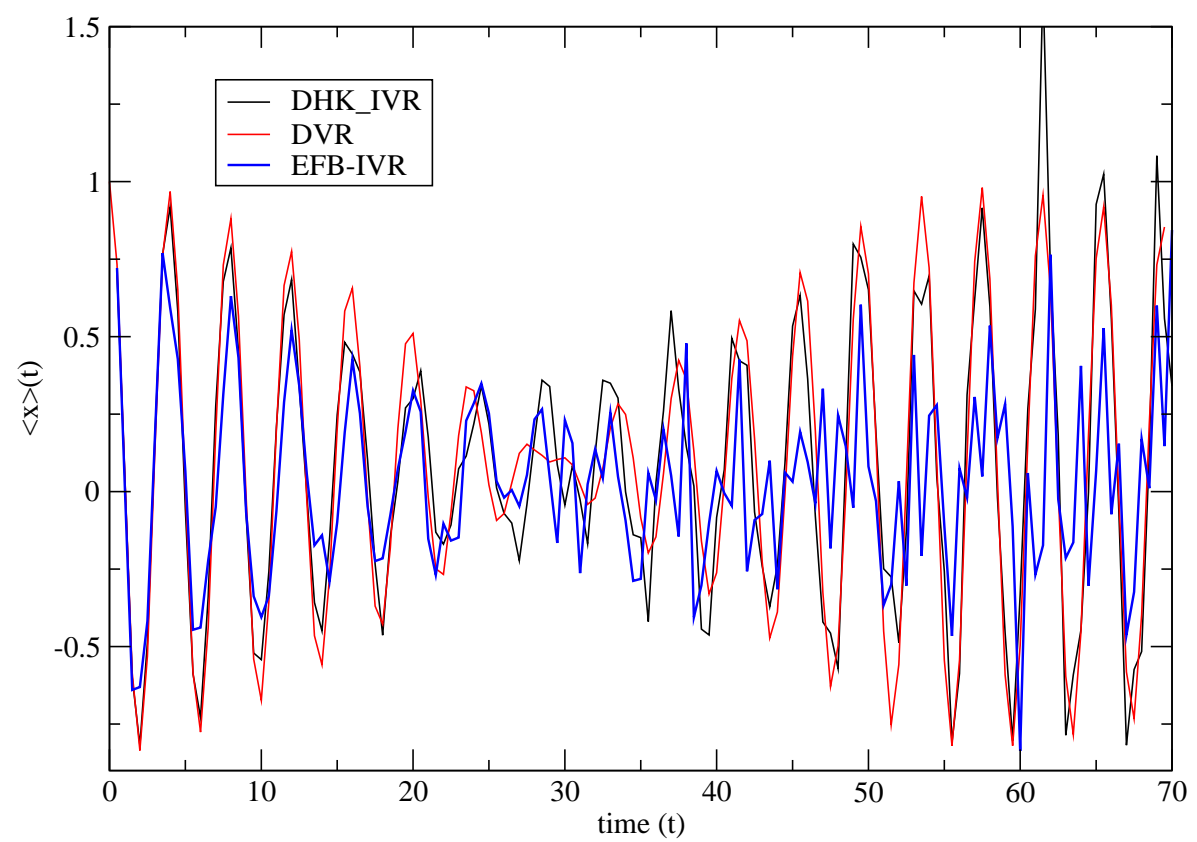

Figure 6.1: Comparison of the position correlation function for an Anharmonic oscillator obtained using DHK-IVR, EFB-IVR and the exact DVR

the oscillation even at short times in both cases as shown in figure Figure 6.2. In Figure 6.3 we show the results of tuning $\mathbf{c}_{\mathbf{p}}$ - and compare how well the different values capture quantum effects. In comparing the results of this calculation with the EFB-IVR we note that it does just as well if not mildly better - this is exactly what we want. The other limit of the calculation is the 'classical' limit and in figure Figure 6.4 we compare the large $\mathbf{c}_{\mathbf{p}}$ limit with a LSC-IVR calculation. The results show that while the behavior is well mimicked, there is still a puzzling fall off in the amplitude which needs further investigation.

We also show the results for a system bath calculation in the limits of $\mathbf{c}_{\mathbf{p}}$ in Figure 6.5. The recurrence effects seem to be well captured in the limit of $\mathbf{c}_{\mathbf{p}}$ being small 


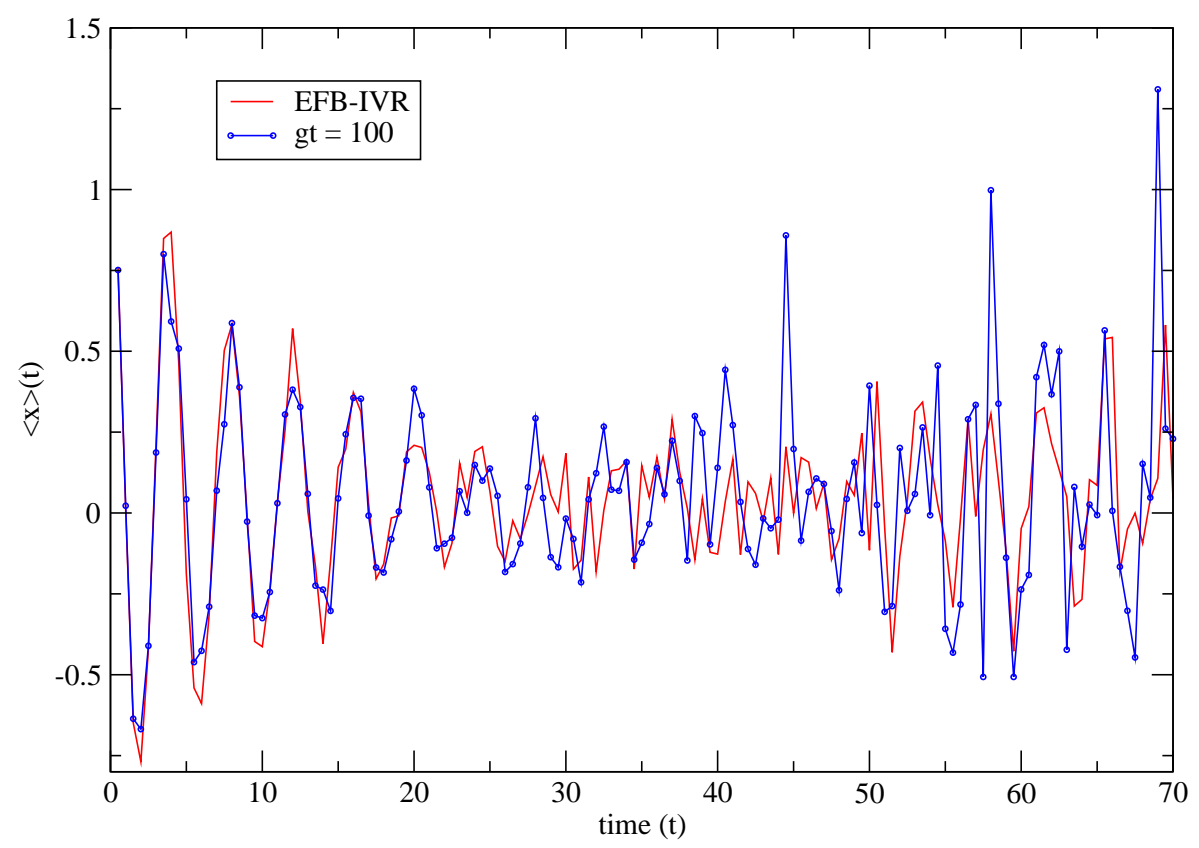

Figure 6.2: Examining the $\gamma_{t} \rightarrow \infty$ limit of the DHK-IVR with the EFB-IVR calculation.

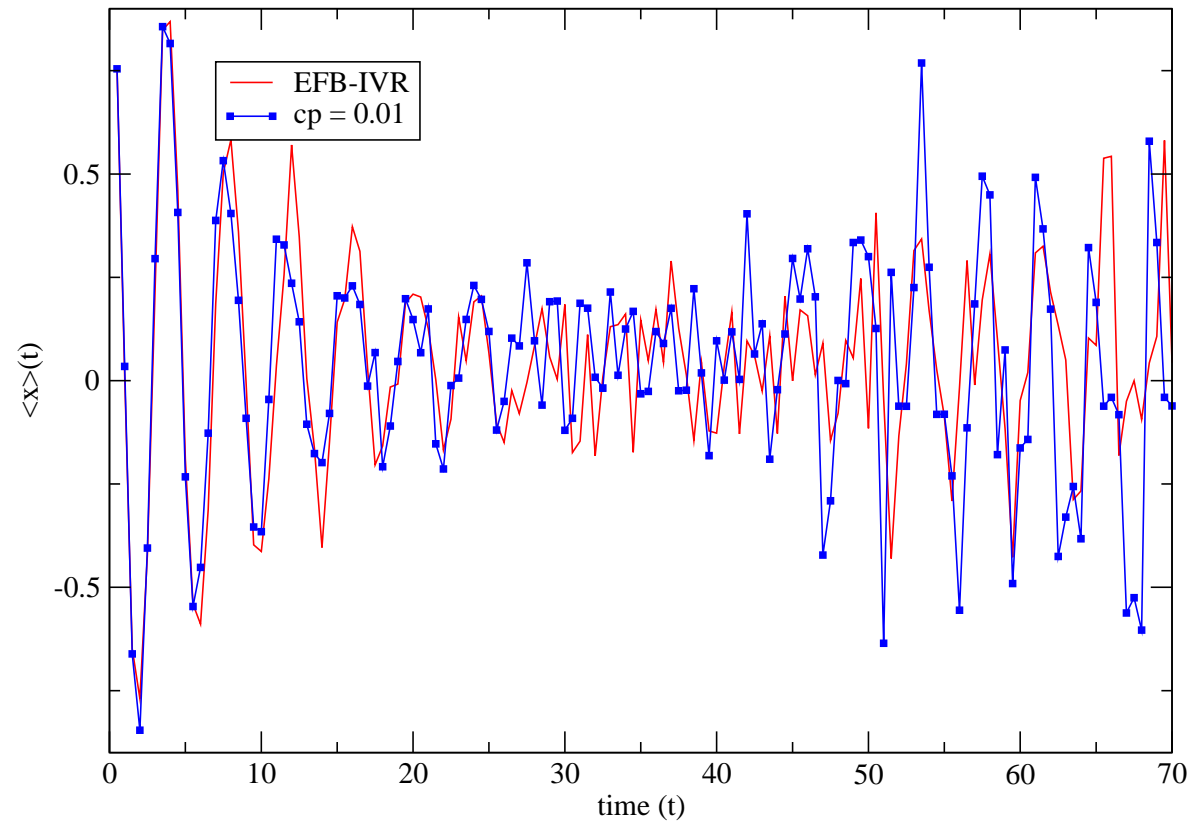

Figure 6.3: The Quantum limit of TIVR - comparing the $\mathbf{c}_{\mathbf{p}} \rightarrow 0$ limit with the EFB-IVR 


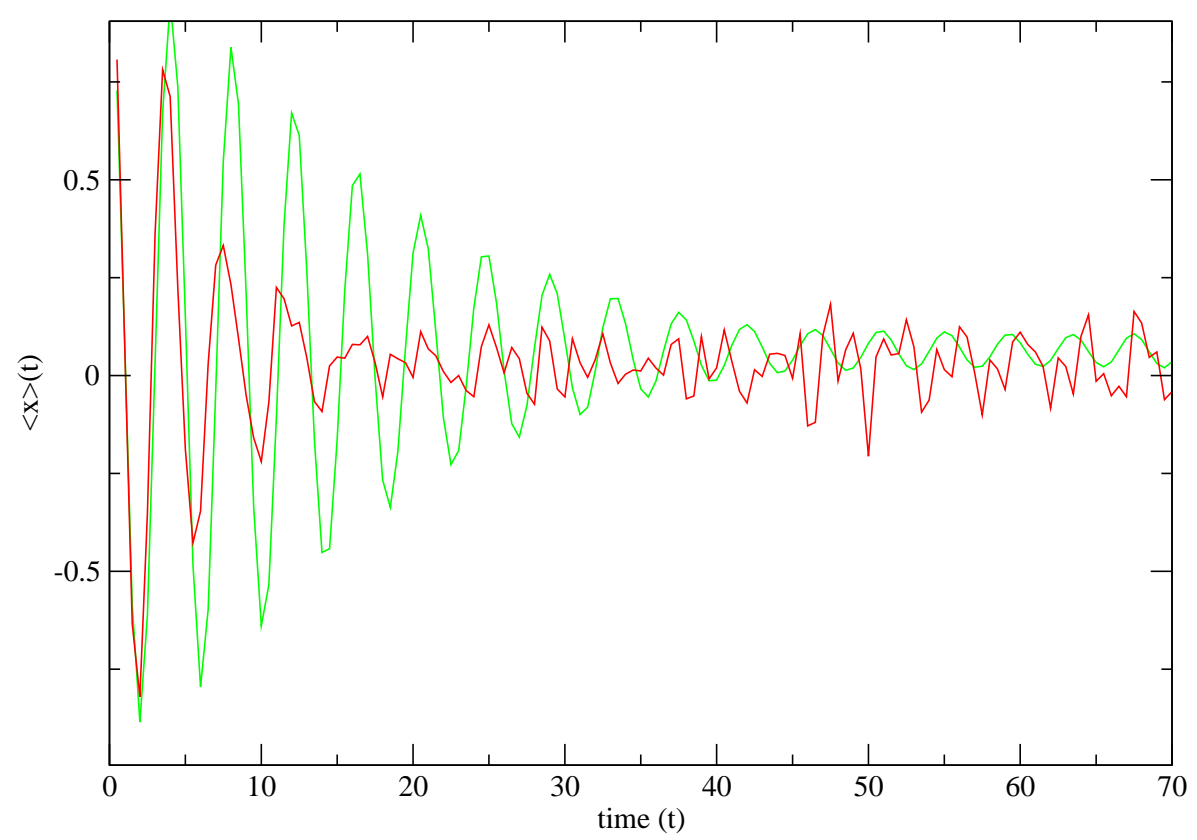

Figure 6.4: The classical limit of the TIVR - contrasting the LSC-IVR result with the limit $\mathbf{c}_{\mathbf{p}} \rightarrow \infty$.

and the problem of a unusually rapid decay in the amplitude of the oscillations appears to be mitigated in this case.

\section{Future Work}

A closer study is needed to make sure that our prefactor tracking technique is indeed legitimate.

One concern with the derivation itself is regarding taking the limits in a multidimensional case. The 1D case is straightforward and merely extending the idea implies that it works the same in higher dimensions, however proving this mathe- 


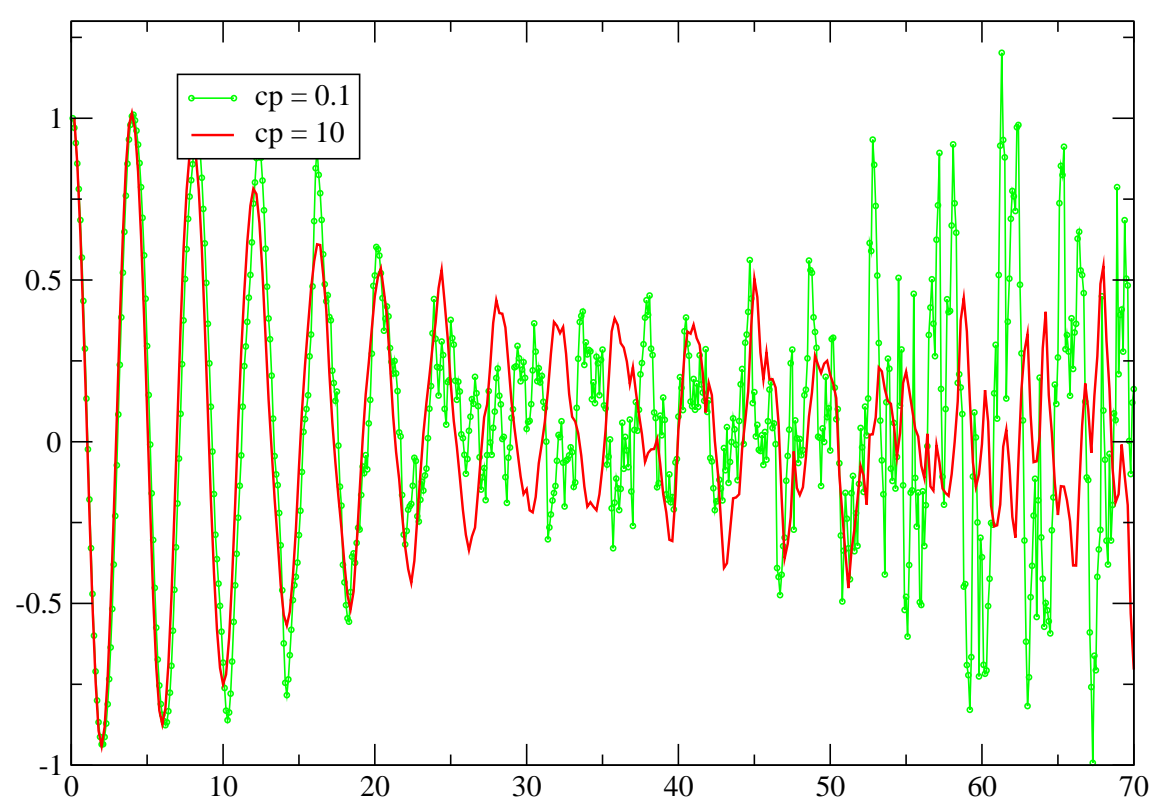

Figure 6.5: The position correlation for an anharmonic system coupled to 10 harmonic bath modes; The limits of a large and a small value of $c p$ are compared.

matically is somewhat trickier.

We would like to understand the physical reasons for the discrepancy between the EFB-IVR and the DHK-IVR which formally at least are equally exact.

Finally, once these details have been ironed out, we would like to study other system bath models which are used to study reaction mechanisms such as proton transfer. 


\section{Chapter 7}

\section{The Semiclassical Prefactor -}

\section{Exploring Alternate Evaluation}

\section{Methods}

\section{Introduction}

Semiclassically, the ultimate goal is to make quantum effects accessible to the Molecular Dynamics community with very little extra effort. The formulation of the SC-IVR for the most part makes use of information obtained from classical trajectories. However, we do require on additional piece of information and this is the Hessian Matrix. Many standard dynamics packages do not provide this de-facto. 
The prefactor in the SC-IVR expression is composed of various Monodromy matrices. The time evolution equations of these matrices involves the Hessian. The obvious way to remove the need for the Hessian is to eliminate the prefactor altogether, like in the LSC-IVR. However, this also reduces the methods ability to capture quantum coherence effects although it can still describe some tunneling and zero point energy effects. Another method to work around needing the Hessian was introduced by Light et $\mathrm{al}^{76}$ where a finite difference scheme (in position and momentum) is proposed to evaluate the Monodromy matrices directly at each time step and by using only the unitarity property of these matrices to carry out time evolution. This is discussed in more detail in the next section.

In this chapter we describe a method to obtain the Hessian by doing a finite difference calculation in time. The idea of such a finite difference is appealing because we have time step information for each trajectory and will therefore, need to do no extra work at all. A near exact scheme is drawn up to accurately calculate the value at each time step and then modified to an approximate scheme with an approximate prefactor that will ideally retain enough accuracy to allow us to obtain quantum effects; This work is ongoing and some preliminary results are shown to describe the progress made so far in documenting the success of this idea. 


\section{Theory}

\section{II.1 Semiclassical Prefactor}

In this first part of the theory section the semiclassical prefactor is described in some detail. The prefactor term in the IVR expression for the time propagator captures the interference between secondary trajectories that are quadratically close to the classical trajectory The expression involves monodromy matrix elements, whose time evolution equations are as follows.

$$
\begin{aligned}
\dot{\mathbf{M}}_{q q} & =\mathbf{M}_{p q} \\
\dot{\mathbf{M}}_{q p} & =\mathbf{M}_{p p}^{f} \\
\dot{\mathbf{M}}_{p q} & =-\frac{\partial^{2} V}{\partial \mathbf{q} \partial \mathbf{q}} \mathbf{M}_{q q} \\
\dot{\mathbf{M}}_{p p} & =-\frac{\partial^{2} V}{\partial \mathbf{q} \partial \mathbf{q}} \mathbf{M}_{q p}
\end{aligned}
$$

The prefactor (for the HK-IVR) takes the form

$C_{t}\left(p_{0}, q_{0}\right)=\operatorname{det}\left|\mathbf{M}_{q q}+\gamma^{-1} \mathbf{M}_{p p} \gamma-i \hbar \mathbf{M}_{q p} \gamma^{-1}+\frac{i}{\hbar} \gamma^{-1} \mathbf{M}_{p q}\right|^{\frac{1}{2}}$

Time evolution of the prefactor involves using the Hessian as well as taking the square root of the resulting complex number. The square root function for a complex number has a branch cut across the negative real axis, so in order to ensure proper continuity in time, we need to track the number of times the value crosses this branch cut. This is relevant to our discussion because this means that 
we need to check for the branch crossing at regular, relatively small time intervals which makes it necessary to also evaluate the Hessian at each of these time steps.

\section{II.2 Log-Derivative Method}

The log-derivative method described in $^{56}$ is primarily introduced as a way to make the evaluation of the prefactor easier. The prefactor in this case is shown to be

$$
C_{t}=\sqrt{\left(\operatorname{det}\left[\frac{1}{2}\left(\mathbf{1}+\frac{\mathbf{i}}{\hbar} \gamma^{-\mathbf{1}} \mathbf{R}_{\mathbf{t}}\right]\right)\right.} e^{\frac{1}{2} \int_{0}^{t} d t^{\prime} \operatorname{Tr}\left(\mathbf{R}_{t}^{\prime}\right)}
$$

where the matrix $\mathbf{R}_{t}$ is defined as

$$
\mathbf{R}_{t}=\dot{\mathbf{Q}}_{t} \mathbf{Q}_{t}^{-1}
$$

The $\mathbf{Q}$ matrix and its time derivative are

$$
\begin{gathered}
\mathbf{Q}=\mathbf{M}_{q q}+\frac{\hbar}{i} \mathbf{M}_{q p} \gamma \\
\dot{\mathbf{Q}}=\mathbf{M}_{p q}+\gamma^{-1} \mathbf{M}_{p p} \gamma
\end{gathered}
$$

It is pretty clear that these two matrices combined form the prefactor defined by Eq. (2.1). The reason for the name 'log-derivative' is also clear from the definition of $\mathbf{R}_{t}$ since

$$
\mathbf{R}_{t}=\frac{\partial \log \mathbf{Q}}{\partial t}=\dot{\mathbf{Q}}_{t} \mathbf{Q}_{t}^{-1}
$$

In practice, the time evolution of $\mathbf{R}$ starts with initial condition

$$
\mathbf{R}_{0}=\frac{\hbar}{i} \gamma
$$


and the equation of motion that it obeys is

$$
\dot{\mathbf{R}}_{t}=-\frac{\partial^{2} V}{\partial \mathbf{q} \partial \mathbf{q}}-\mathbf{R}_{t}^{2}
$$

The prefactor has a branch cut discontinuity when the real part becomes negative and the imaginary part goes through zero. It has been that the branch cut conditions for a prefactor of the form in Eq. (2.1) correspond to

$$
\begin{aligned}
1+\frac{1}{\mathbf{M}_{q q}^{2}+(\hbar \gamma) \mathbf{M}_{q p}^{2}} & <0 \\
\mathbf{M}_{p q} \mathbf{M}_{q q} & =-(\hbar \gamma)^{2} \mathbf{M}_{p q} \mathbf{M}_{q p}
\end{aligned}
$$

In one-dimension, the condition in Eq. (2.7) is never true since the left hand side is always positive; this means that the prefactor simply does not have a branch cut. In multidimensional problems, this is not as immediately obvious. Several systems were tested and it seems likely (although not a rigorous truth) that a negative real part occurring in conjunction with a vanishing imaginary part is an unlikely scenario.

An approximation to the exact prefactor in Eq. (2.1) can be made by assuming that the matrix $\mathbf{R}_{t}$ is slowly varying, or in other words, by setting

$$
\dot{\mathbf{R}}_{t}=0
$$

This leads to Johnson's multichannel WKB prefactor which has the form

$$
C_{t} \approx e^{-\frac{i}{\hbar} \int_{0}^{t} d t^{\prime} \sum_{j=1}^{N} \frac{1}{2} \hbar \omega_{j}\left(t^{\prime}\right)}
$$


where $\omega_{j}\left(t^{\prime}\right)^{2}$ are the eigenvalues of the Hessian matrix.

In the next few sections of this chapter, we describe methods to obtain the Hessian by finite difference and use this, in some cases, approximate Hessian to obtain the prefactor either with the log derivative or even the WKB method. While such a procedure will definitely be non-exact, there is still a high likelihood that it will prove a good enough to still capture quantum effects such as coherence.

\section{II.3 Finite Difference - Light Method}

The stability matrix $\mathbf{M}\left(t_{1}, t_{2}\right)$ of a given trajectory is the jacobian of the transformation from the point $\left(\mathbf{q}_{\mathbf{1}}, \mathbf{p}_{\mathbf{1}}\right)$ at $t_{1}$ to $\left(\mathbf{q}_{\mathbf{2}}, \mathbf{p}_{\mathbf{2}}\right)$ at time $t_{2}$ under the system Hamiltonian $\hat{H}$. The stability matrix is composed of monodromy matrices in the form

$$
M\left(t_{1}, t_{2}\right)=\left[\begin{array}{cc}
\frac{\partial \mathbf{p}_{2}}{\partial \mathbf{p}_{1}} & \frac{\partial \mathbf{p}_{2}}{\partial \mathbf{q}_{1}} \\
\frac{\partial \mathbf{q}_{2}}{\partial \mathbf{p}_{1}} & \frac{\partial \mathbf{q}_{2}}{\partial \mathbf{q}_{1}}
\end{array}\right]
$$

This stability Matrix time evolves as per the equation

$$
\frac{\mathbf{M}\left(\mathbf{t}_{\mathbf{1}}, \mathbf{t}_{\mathbf{2}}\right)}{d t}=\left(\begin{array}{cc}
0 & \frac{\partial^{2} H}{\partial \mathbf{q} \partial \mathbf{q}} \\
\frac{\partial^{2} H}{\partial \mathbf{p} \partial \mathbf{p}} & 0
\end{array}\right) \mathbf{M}\left(t_{1}, t_{2}\right)
$$

The Unitarity property of the stability matrix allows the use of the chain rule, which gives us

$$
\left.\mathbf{M}\left(\mathbf{t}_{\mathbf{3}}, \mathbf{t}_{\mathbf{1}}\right)=\mathbf{M}\left(\mathbf{t}_{\mathbf{3}}, \mathbf{t}_{\mathbf{2}}\right) \cdot \mathbf{M}\left(\mathbf{t}_{\mathbf{2}}, \mathbf{t}_{\mathbf{1}}\right)\right)
$$


where $M\left(t, t^{\prime}\right)$ indicates the monodromy matrix for time evolution between time $t^{\prime}$ and $t$. This property allows us to start with initial conditions for the matrix $\mathbf{M}$ at any intermediate time.

At each time, there is a central trajectory

$$
\mathbf{z}_{0}(\mathbf{t})=\mathbf{q}_{1}(\mathbf{t}), \mathbf{q}_{2}(\mathbf{t}), \ldots, \mathbf{q}_{\mathbf{N}}(\mathbf{t}), \mathbf{p}_{\mathbf{1}}(\mathbf{t}), \mathbf{p}_{\mathbf{2}}(\mathbf{t}), \ldots, \mathbf{p}_{\mathbf{N}}(\mathbf{t})
$$

At each time, a partial differentiation is performed numerically. In order to do this, an additional $2 \mathrm{~N}$ trajectories are propagated, with initial conditions that differ from $\mathbf{z}_{\mathbf{0}}(\mathbf{t})$ by a small displacement in one of the variables.For instance,

$$
\begin{aligned}
\mathbf{z}_{\mathbf{1}}(\mathbf{t}) & =q_{1}(t)+\Delta q_{1}, q_{2}(t), \ldots, q_{N}(t), p_{1}(t), p_{2}(t), \ldots p_{N}(t) \\
\mathbf{z}_{\mathbf{N}+\mathbf{1}}(\mathbf{t}) & =q_{1}(t), q_{2}(t), \ldots, q_{N}(t), p_{1}(t)+\Delta p, p_{2}(t), \ldots p_{N}(t) \\
\mathbf{z}_{\mathbf{2 N}}(\mathbf{t}) & =q_{1}(t), q_{2}(t), \ldots q_{N}(t) p_{1}(t), p_{2}(t), \ldots p_{N}(t)+\Delta p
\end{aligned}
$$

The elements $M_{i j}$ of the matrix $\mathbf{M}$ are then given by

$$
\begin{aligned}
M_{i j} & =\frac{\partial z^{i}(t+\Delta t)}{\partial z^{j}(t)} \\
& =\frac{z_{j}^{i}(t+\Delta t)-z_{0}^{i}(t+\Delta t)}{\Delta z_{j}}
\end{aligned}
$$

This methods has proven to be quite accurate and by decreasing the size of the time steps or by using $4 \mathrm{~N}$ trajectories with displacements $\pm \frac{\Delta q}{2}$ and $\pm \frac{\Delta p}{2}$ which 
will allows us to use a central difference scheme for the derivatives. The main advantage of this method is that there is no longer any need for the Hessian. The drawbacks is the number of additional trajectories required at every time step. We would like a method that has all the advantages of the current one and one more - the fact that no extra trajectories are required.

\section{II.4 Finite Difference in Time}

As described in the previous section, the Light method is one way to obtain the Monodromy matrices in time and hence the prefactor without any need for the Hessian. Our current task is to describe another such finite difference scheme, but instead of the Monodromy matrices, we look at obtaining the Hessian.

Consider the equation for time evolution of momentum

$$
\dot{p}=\left(-\frac{d V}{d q}\right)
$$

We can now take the time derivative of both sides of the equation

$$
\frac{\partial^{2} \mathbf{p}}{\partial t \partial t}=-\left(\frac{\partial^{2} V}{\partial q \partial q}\right) \dot{q}
$$

We can substitute this for

$$
\dot{\mathbf{q}}=\frac{\partial H}{\partial \mathbf{p}}=\frac{\mathbf{p}}{m}
$$

This makes our exression

$$
\frac{\partial^{2} p}{\partial t \partial t}=-\left(\frac{\partial^{2} V}{\partial q \partial q}\right) \frac{p}{m}
$$


This is the final expression we will be working with. It resembles a standard matrix equation, but with one twist that makes things harder! A regular matrix equation takes the form,

$$
\mathbf{A} \vec{x}=\vec{y}
$$

where $\mathbf{A}$ is a matrix and the others are vectors. In such an expression, $\vec{x}$ is usually the unknown. This means that to solve the equation, we simply invert matrix $\mathbf{A}$ and evaluate

$$
\vec{x}=[\mathbf{A}]^{-1} \vec{y}
$$

In the case of the Hessian, however, the matrix $\frac{\partial^{2} V}{\partial q \partial q}$ is the unknown. Solving the equation for the matrix, is somewhat more complicated!

We consider two ways to work around this. One way is to do some kind of Singular Value Decomposition (SVD) which effectively gives us the eigenvalues that can then be used to find the inverse rectangular matrix (i.e. a vector $\vec{x} !)$. While SVD is quite effective in some cases, it is clear that here we simply do not have enough information to fully obtain all the elements of the Hessian matrix with any degree of accuracy.

The other approach is to construct $\vec{x}$ and $\vec{y}$ such that they are actually matrices not vectors and therefore invertible! The matrix form of $\vec{x}$ is simply a matrix of the positions at consecutive time steps. If we store the position at as many different time steps as there are degrees of freedom, we have a square matrix that is invertible as long as its determinant is non-zero. 
One assumption that is built into such a procedure is that the Hessian is constant over the time steps which we use to construct our matrix. Clearly, for systems with many degrees of freedom, the approximation will begin to break down if we have to use a correspondingly large number of time steps. So we look around for some way to reduce the number of time steps used in constructing the matrix while still being able to determine the Hessian

With this in mind, we start by looking at the simplest case where the Hessian is diagonal. Our matrix equation can now be written as

$$
\sum_{k^{\prime}=1}^{N} A_{k k^{\prime}} x_{k^{\prime} i}=y_{k i}
$$

if $A_{k k^{\prime}}=a_{k k^{\prime}} \delta_{k k^{\prime}}$ then the equation reduces to

$a_{k k} x_{k i}=y_{k i}$ which is a really simple equation to solve for $a_{k k}$.

We can look at a less extreme case where the Hessian is block diagonal and there are $M$ near-diagonals that have non-zero elements other than the primary diagonal. We can treat such a case in a fashion similar to the pure diagonal case

$$
\sum_{k^{\prime}=k-M}^{k+M} A_{k k^{\prime}} x_{k^{\prime} i}=y_{k i}
$$

The values of $k^{\prime}$ in this case run from $k-M$ to $k+M$, a total of $2 M+1$ values and i represents the $i^{\text {th }}$ degree of freedom.

For a given value of $k, A_{k k^{\prime}}$ is a vector of length $2 M+1$ and if we choose the same number of values for $i$ the $x$ matrix is then square and $y_{k i}$ is a vector. The solution, 
is then,

$$
A_{k k^{\prime}}=\sum_{i=1}^{N} y_{k i}\left(x^{-1}\right)_{i k^{\prime}}
$$

where we simply invert the $2 M+1$ dimensional $\mathrm{x}$ matrix which will in general be less than $N$ if our Hessian is block diagonal in nature.

\section{Discussion}

We start studying our new technique with the easiest model - the 1D case is trivial and exact. This is a good way to check how well our technique does with the assumption that the Hessian is constant over a finite period of time. We study a Morse potential of the form,

$$
\hat{H}_{M O}=\frac{1}{2} p^{2}+D\left(e^{-2 \alpha q}-2 e^{-\alpha q}\right)
$$

The parameters chosen are $\alpha=0.67 a u^{-1}$ and $D=4 a u$. The initial condition for the trajectory are chosen to be $q_{0}=-0.35 \mathrm{au}$ and $p_{0}=0$.

As expected, our results are near exact for the prefactor and careful testing shows that in this case at least, the size of the time step over which the Hessian is assumed to be constant can be several times the trajectory time step Figure 7.1 shows the HK-IVR prefactor obtained from an analytically calculated Hessian compared with one calculated from a finite difference Hessian and a log-derivative prefactor that also used the calculated Hessian. We now move on to our next testing grounds - a 2D model that consists of two linearly coupled anharmonic oscillators. The 


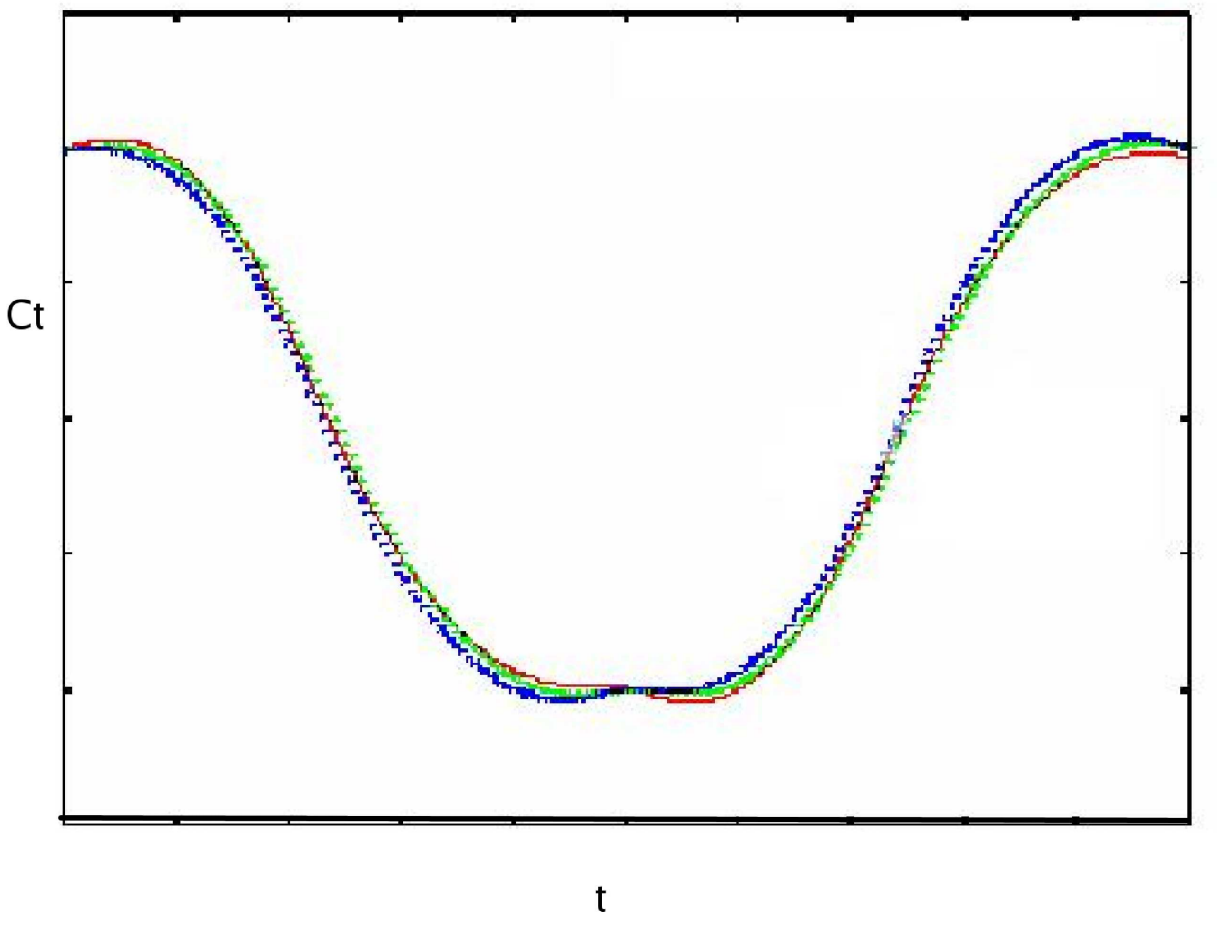

Figure 7.1: The prefactor for a simple 1D Morse potential; the red line is the HK prefactor with an analytic Hessian; the blue and green dotted lines are the log-derivative and HK prefactors respectively with a finite difference Hessian. 
potential is of the form

$$
V\left(x_{1}, x_{2}\right)=\frac{1}{2}\left(x_{1}^{2}+x_{2}^{2}\right)+\frac{1}{4}\left(x_{1}^{4}+x_{2}^{4}\right)+x_{1} x_{2}
$$

This potential is chosen as a good balance between sufficiently anharmonic to provide a challenge and smooth enough to make the slowly varying Hessian approximation reasonable.

Figures 7.2 and 7.3 compare the diagonal and off-diagonal elements of the two dimensional Hessian. To understand this, we look at a 1D version and note

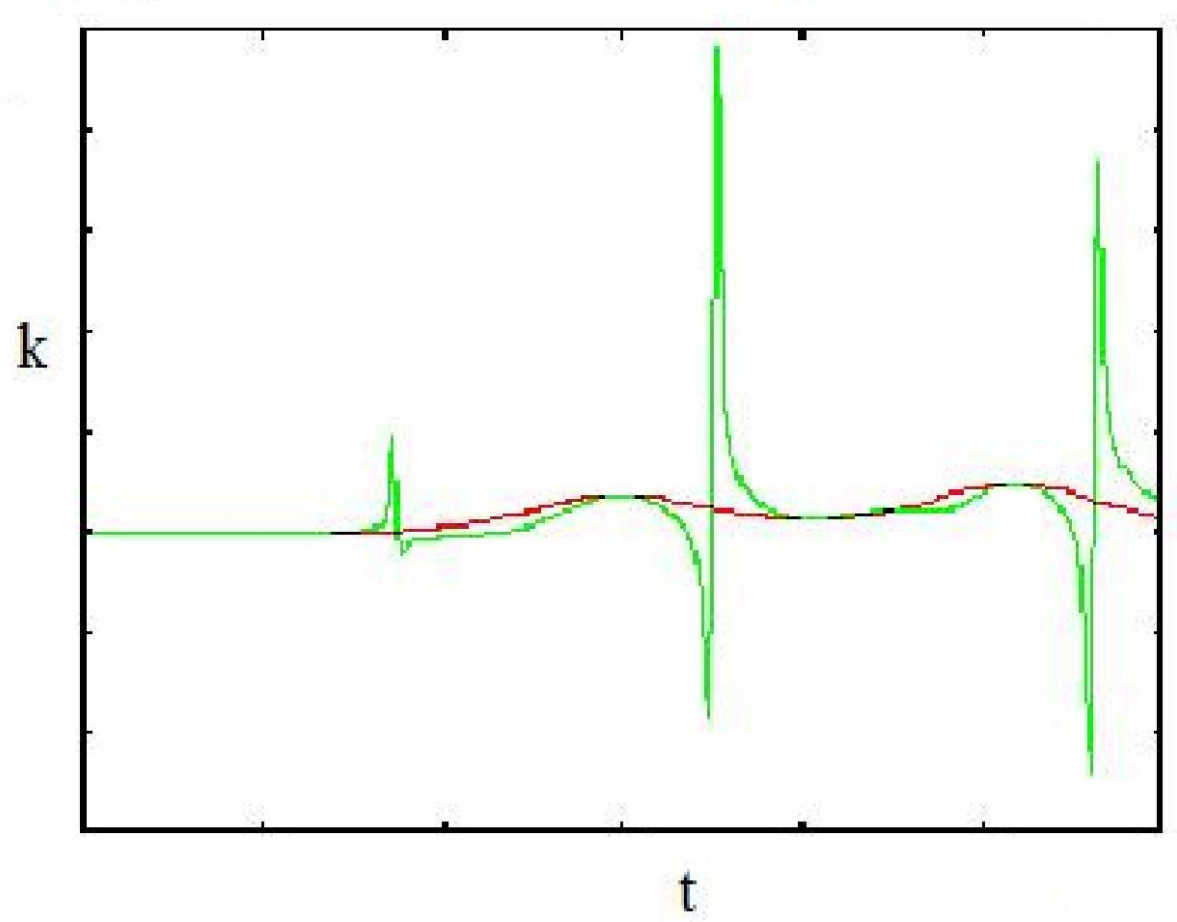

Figure 7.2: Comparing the diagonal elements of the analytic(red) Hessian and the finite difference (green) Hessian.

that we see similar behavior in the Hessian. The calculated Hessian mimics the 


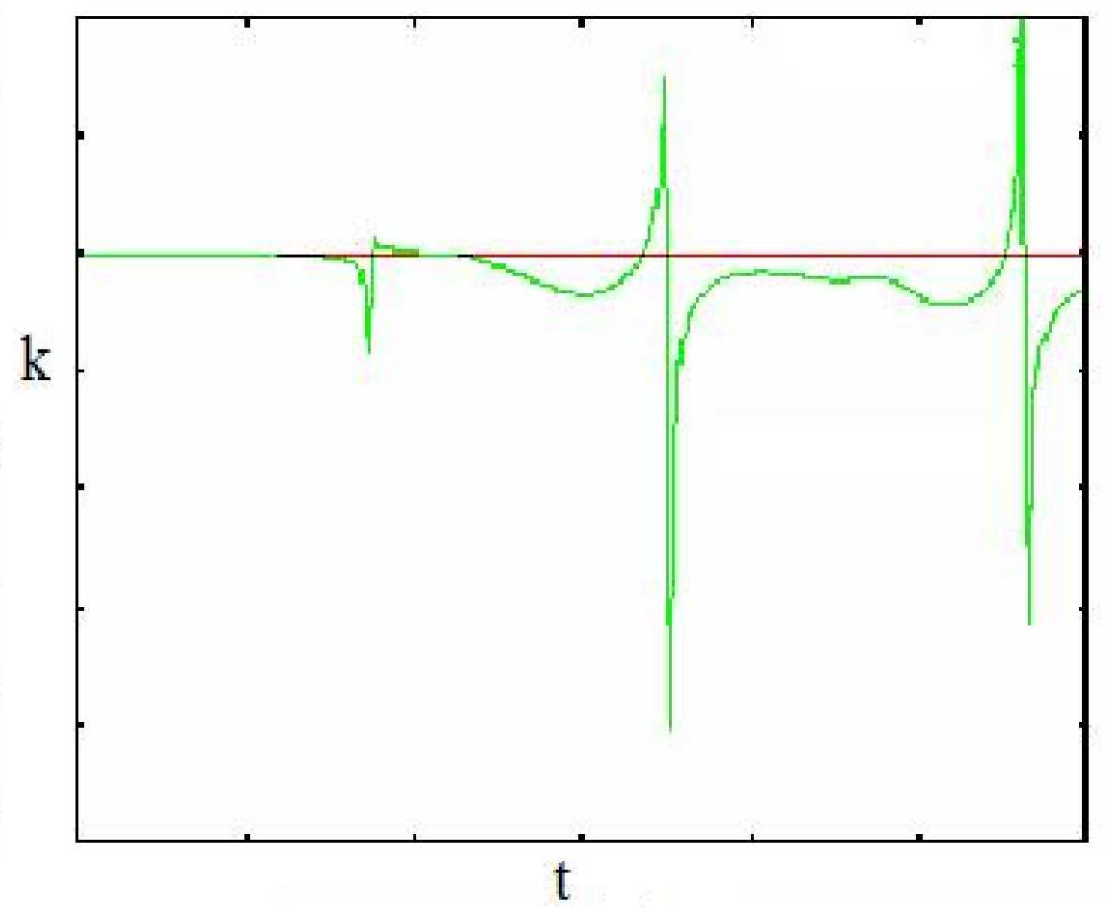

Figure 7.3: Comparing the off-diagonal elements of the analytic(red) Hessian and the finite difference (green) Hessian.

analytical Hessian for the most part, but at various points we encounter what appear to be points of near-singularity.

These actually arise from the momentum values becoming small. In $1 \mathrm{D}$ this problem is somewhat mitigated by the fact that we are dividing two small numbers and hence the Hessian does not become too large as shown in Figure 7.4 This is actually something we believe we can successfully work around now that the problem has been identified (literally, by stepping over these points!). In many dimensions, the relevant quantity to watch for is the determinant of the matrix to be inverted. 


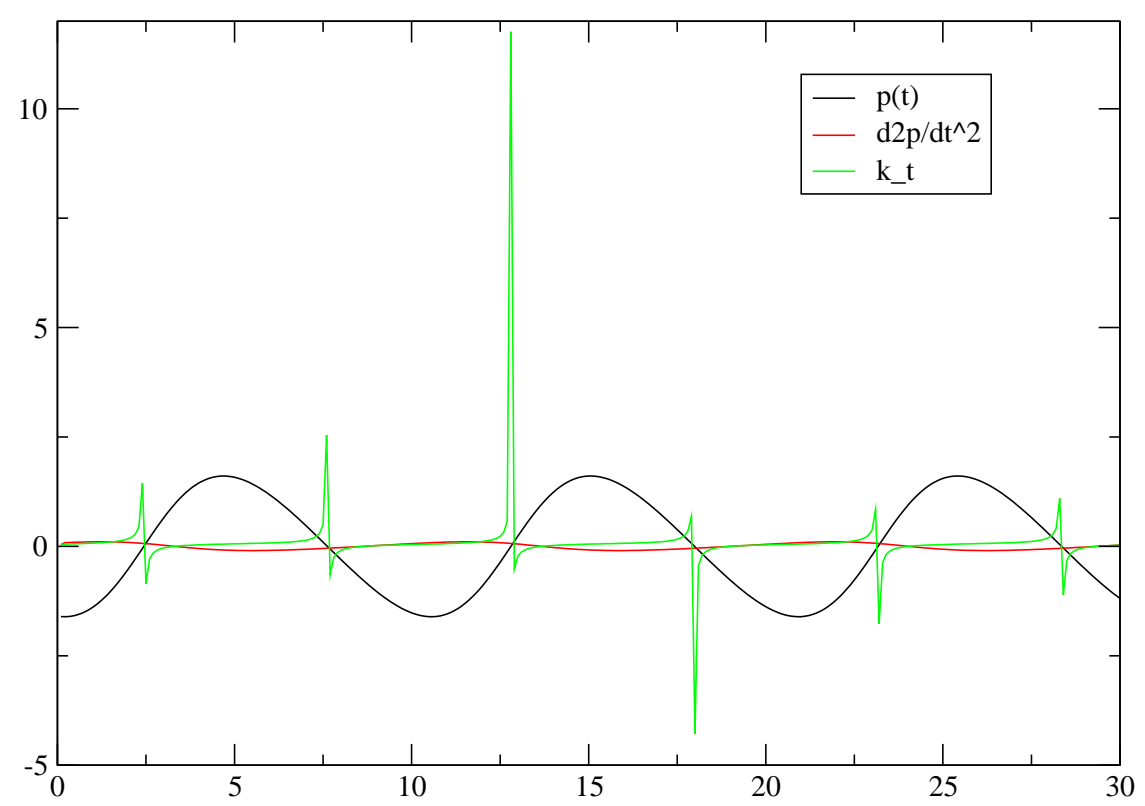

Figure 7.4: The momentum, the second derivative of the momentum in time and the force constant along the course of the trajectory

One last calculation we describe here is the use of the finite difference scheme with the WKB approximation for the prefactor. We wish to investigate this combination specifically because if successful, we have worked around both the computational difficulties associated with the calculation of the prefactor. Namely, we will not require the Hessian and we will not have to track the Maslov Index.

We do this calculation for a rather more anharmonic Morse potential as a tough case. The potential is of the form

$$
V(x)=D\left(1-e^{\lambda x}\right)^{2}
$$

and the parameters are $D=30 a . u$. and $\lambda=0.08 a . u$. We also use a more excited state wavefunction in the HK-IVR calculation with a coherent state width $\gamma=12$. 
The autocorrelation function for such a morse potential is calculated and we compare the exact results with the WKB results obtained both by using the analytic Hessian for the prefactor and our finite difference scheme for the prefactor. The results are shown in Figure 7.5. We see that the WKB is not very accurate here, the results being lower than expected, however there is little to choose between using the analytic Hessian and the Finite difference Hessian which, again, was expected in a one-dimensional model.

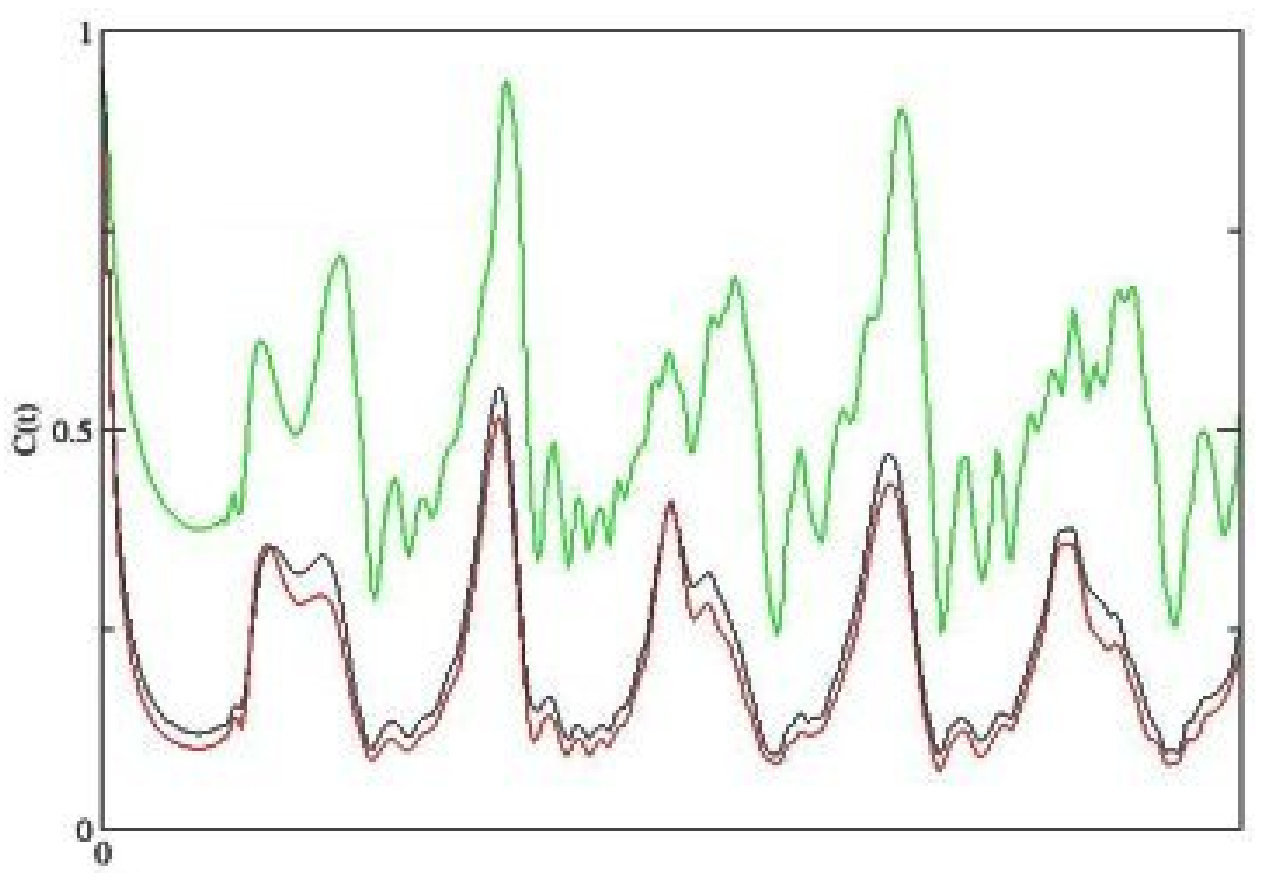

Figure 7.5: The autocorrelation function using the regular HK-IVR prefactor, and the WKB prefactor calculated with the analytic Hessian(black) and the finite difference Hessian (red). 


\section{Future Work}

Work is currently underway on several fronts here. We believe that this method is most promising and are investigating several variants on this, including carrying out a finite difference in the position along the trajectory by merely storing three steps at a time.

We are also on the lookout for a realistic system with a block diagonal Hessian that we can try the equations described for just such as case Eq. (2.24). 


\section{Chapter 8}

\section{Conclusion}

In this chapter we summarize the work described in the previous chapters to gain a little perspective on the goals of this body of research. Semiclassical methods such as the IVR possess the great advantage of being rigorously derivable and therefore provide a solid foundation which we can then improve upon or approximate as per our needs. In the context of modern research, despite the fact that there are many things we do not still understand about small molecules and gas phase dynamics, interest is definitely shifting towards understanding larger, more complex molecules and condensed phase dynamics. The SC-IVR can, in theory, describe this entire range of dynamics but several practical problems begin to play up as soon as we move to higher dimensional systems.

The sign problem arising due to the oscillatory nature of the integrand is one. In this thesis, the TIVR addresses this problem. The solution proposed is to 
choose only a few degrees of freedom which will contribute to the oscillatory nature of the integrand and let all the others cancel out exactly. The action term in the exponent is the sum of the forward and backward contributions and in the classical limit simulation these will be identical. In essence the classical limit of the Filinov parameter corresponds (as described before) to a stationary phase approximation. We believe that this methodology will help minimize the sign problem since entirely eliminating the phase factor (as we do in the LSC-IVR) means foregoing all coherence effects.

Another problem is the computational cost that comes with scaling up. Calculating the prefactor is a major bottleneck as described in the previous chapter. The 'dream team' solution here would be if we could compute the Hessian with only the standard, existing classical trajectory information and then use the log-derivative or the somewhat cruder WKB approximation for the prefactor to avoid tracking the Maslov index. We are currently investigating several such avenues.

We now move on to the more application oriented parts of this thesis. It has been shown that the IVR formalism lends itself to the accurate calculation of energy eigenvaluse, although the implementation needs refinement. Nonadiabatic dynamics are becoming more and more important in the modern context. It is clear that curve crossings and conical intersection are the norm rather than the exception and being able to build a consistent dynamic model to deal with such events is very important. We believe that the MMST hamiltonian in conjunction 
with the FB-IVR is just such a method.

Finally, we describe an algorithm to improve the implementation of the IVR. We show clearly, where time independent sampling falls short in evaluating long time dynamics of a system. Time dependent sampling is usually avoided because of the high cost involved and in the case of parallel computing the high communication needed between processors. We describe a scheme that addresses and somewhat mitigates both problems. The only additional work lies in running a pure classical trajectory simulation over and above the usual one. As far as communication requirements, the sampling algorithm is such that there is no need for the different processors to compare notes, all the information required is locally available in the form of the classical trajectories run on each individual processor.

Refinements such as the ones described here and elsewhere are part of the ongoing process to make semiclassical methods a viable tool to study and predict the dynamics in complex systems to a hight degree of accuracy. The extra insight that one gets from looking at quantum effects from the viewpoint of a classical limit theory also makes it important to continue work in this area. 


\section{Bibliography}

[1] G. Wentzel. Eine Verallgemeinerung der Quantenbedingungen für die Zwecke der Wellenmechanik. Z. Phys, 38(6):518-529, 1926.

[2] HA Kramers. Wellenmechanik und halbzahlige Quantifizierung. Z. Phys, 39:828-840, 1926.

[3] L. Brillouin. La mecanique ondulatoire de Schrodinger; une methode generale de resolution par approximations successives. CR Acad. Sci, 183(11):24-26, 1926.

[4] M. Born, D.R. Hartree, and J.W. Fisher. The Mechanics of the atom. New York: Ungar, 1960.

[5] M.V. Berry and KE Mount. Semiclassical approximations in wave mechanics. Rep. Prog. Phys., 35(1):315-397, 1972.

[6] IC Percival. Regular and irregular spectra. J. Phys. B, 6, 1973. 
[7] A. Einstein. Zum quantensatz von Sommerfeld und Epstein. Verh. Dtsch. Phys. Ges., 19:82-92, 1917.

[8] L. Brillouin. Remarques sur la mecanique ondulatoire. J. Phys. Radium, 7(12):353-368, 1926.

[9] J.B. Keller. Corrected bohr-sommerfeld quantum conditions for nonseparable systems. Ann. Phys., 4(2):180-188, 1958.

[10] JH van Vleck. The Correspondence Principle in the Statistical Interpretation of Quantum Mechanics. Proc. Natl. Acad. Sci., 14(2):178-188, 1928.

[11] MC Gutzwiller and J. Math. Phys. 8, 1979-2000 (1967). J. Math. Phys, 12:343-358, 1971.

[12] R.P. Feynman and A.R. Hibbs. Quantum mechanics and path integrals. McGraw-Hill New York, 1965.

[13] V.P. Maslov and MV Fedoriuk. Semi-Classical Approximation in Quantum Mechanics. Kluwer Academic Publishers, 2002.

[14] R.G. Littlejohn. The Van Vleck formula, Maslov theory, and phase space geometry. J. Stat. Phys., 68(1):7-50, 1992.

[15] Note. A caustic is typically a point where the momentum changes direction or the velocity becomes identically zero in the course of the trajectory. 
[16] W.H. Miller. Classical-limit quantum mechanics and the theory of molecular collisions. Adv. Chem. Phys, 25:69, 1974.

[17] R.G. Littlejohn. The semiclassical evolution of wave packets. Phys. Rep., 138(4-5):193-291, 1986.

[18] E.J. Heller. Time-dependent approach to semiclassical dynamics. J. Chem. Phys., 62:1544, 1975.

[19] E.J. Heller. Frozen Gaussians: A very simple semiclassical approximation. J. Chem. Phys., 75:2923, 1981.

[20] W.H. Miller. The semiclassical initial value representation: A potentially practical way for adding quantum effects to classical molecular dynamics simulations. J. Phys. Chem. A, 105(13):2942-2955, 2001.

[21] M. Thoss and H. Wang. Semiclassical description of molecular dynamics based on initial-value representation methods. Annu. Rev. Phys. Chem., 55:299-332, 2004 .

[22] William H. Miller. Classical s matrix: Numerical application to inelastic collisions. J. Chem. Phys., 53(9):3578-3587, 1970.

[23] R. A. Marcus. Theory of semiclassical transition probabilities (s matrix) for inelastic and reactive collisions. uniformization with elastic collision trajectories. J. Chem. Phys., 56(7):3548-3550, 1972. 
[24] M.F. Herman. Improving the accuracy of semiclassical wavepacket propagation using integral conditioning techniques. Chem. Phys. Lett., 275(5-6):445$452,1997$.

[25] G. Campolieti and P. Brumer. Semiclassical propagation: Phase indices and the initial-value formalism. Phys. Rev. A, 50(2):997-1018, 1994.

[26] K.G. Kay. Semiclassical initial value treatments of atoms and molecules. Annu. Rev. Phys. Chem, 56:255, 2005.

[27] S. Tomsovic and E.J. Heller. Semiclassical dynamics of chaotic motion: Unexpected long-time accuracy. Phys. Rev. Lett., 67(6):664-667, 1991.

[28] M.L. Brewer, J.S. Hulme, and D.E. Manolopoulos. Semiclassical dynamics in up to 15 coupled vibrational degrees of freedom. J. Chem. Phys., 106:4832, 1997.

[29] DJ Tannor and S. Garashchuk. Semiclassical calculation of chemical reaction dynamics via wavepacket correlation functions. Annu. Rev. Phys. Chem., 51:553-600, 2000.

[30] R. Hernandez and G.A. Voth. Quantum time correlation functions and classical coherence. Chem. Phys., 233(2-3):243-255, 1998.

[31] Roy J. Glauber. Coherent and incoherent states of the radiation field. Phys. Rev., 131(6):2766-2788, Sep 1963. 
[32] E. Herman M.F, Kluk. A semiclassical justification for the use of nonspreading wavepackets in dynamics calculations. Chem. Phys., 91(1):27-34, 1984.

[33] K.G. Kay. Integral expressions for the semiclassical time-dependent propagator. J. Chem. Phys., 100:4377, 1994.

[34] W.H. Miller. An Alternate Derivation of the Herman-Kluk(Coherent State) Semiclassical Initial Value Representation of the Time Evolution Operator. Mol. Phys., 100:397-400, 2002.

[35] S.A. Deshpande and G.S. Ezra. On the derivation of the Herman-Kluk propagator. J. Phys. A: Math. Gen, 39:5067-5078, 2006.

[36] W.H. Miller. Including quantum effects in the dynamics of complex (ie, large) molecular systems. J. Chem. Phys., 125:132305, 2006.

[37] Xiong Sun and William H. Miller. Forward-backward initial value representation for semiclassical time correlation functions. J. Chem. Phys., 110(14):6635-6644, 1999.

[38] Haobin Wang, Michael Thoss, Kathy L. Sorge, Ricard Gelabert, Xavier Giménez, and William H. Miller. Semiclassical description of quantum coherence effects and their quenching: A forward-backward initial value representation study. J. Chem. Phys., 114(6):2562-2571, 2001. 
[39] X. Sun, H. Wang, and W.H. Miller. On the semiclassical description of quantum coherence in thermal rate constants. J. Chem. Phys., 109:4190, 1998.

[40] X. Sun, H. Wang, and W.H. Miller. Semiclassical theory of electronically nonadiabatic dynamics: Results of a linearized approximation to the initial value representation. J. Chem. Phys., 109:7064, 1998.

[41] W.H. Miller. Generalization of the linearized approximation to the semiclassical initial value representation for reactive flux correlation functions. J. Phys. Chem. A, 103(47):9384-9387, 1999.

[42] E. Pollak and J.L. Liao. A new quantum transition state theory. J. Chem. Phys., 108:2733, 1998.

[43] J.A. Poulsen, G. Nyman, and P.J. Rossky. Practical evaluation of condensed phase quantum correlation functions: A Feynman-Kleinert variational linearized path integral method. J. Chem. Phys., 119:12179, 2003.

[44] William H. Miller. TEX: Eigenvalues and Reaction Rate Constants, volume Recent Developments in Semiclassical Mechanics of The New World of Quantum Chemistry. D.Reidel Publishing Co., Dordrecht, Netherlands, 1976.

[45] Sally Chapman, Bruce C. Garrett, and William H. Miller. Semiclassical eigenvalues for nonseparable systems: Nonperturbative solution of the hamilton- 
jacobi equation in action-angle variables. J. Chem. Phys., 64(2):502-509, 1976.

[46] Ronnie Kosloff. Time-dependent quantum-mechanical methods for molecular dynamics. J. Phys. Chem., 92(8):2087-2100, 1988.

[47] William H. Miller Srihari Keshavamurthy. Semiclassical correction for quantum-mechanical scattering. Chem. Phys. Lett., 218(3):189-194, 1994.

[48] E. Merzbacher. Quantum mechanics. Wiley [New York, 1998.

[49] L. Brillouin. Les problãmes de perturbations et les champs self-consistents. J. Phys., 3:373, 1932.

[50] EP Wigner. Math. naturw. Anz. ungar. Akad. Wiss, 53:475, 1935.

[51] Anna Okopińska. Accurate energy levels and partition function of a quantummechanical anharmonic oscillator. Phys. Rev. D, 36(4):1273-1275, Aug 1987.

[52] Neil R. Kestner and Oktay Sinanoḡlu. Study of electron correlation in heliumlike systems using an exactly soluble model. Phys. Rev., 128(6):2687-2692, Dec 1962.

[53] Randall W. Hall. Comparison of path integral and density functional techniques in a model two-electron system. J. Phys. Chem, 93(14):5628-5632, 1989. 
[54] D.T. Colbert. A novel discrete variable representation for quantum mechanical reactive scattering via the S-matrix Kohn method. J. Chem. Phys., 96(3):1982, 1992.

[55] Li Yimin. The work in this chapter is based on yimin li's ideas and the calculation using a 3d water potential was entirely carried out by him. 2006 .

[56] R. Gelabert, X. Gimenez, M. Thoss, H. Wang, and W.H. Miller. A logderivative formulation of the prefactor for the semiclassical herman-kluk propagator. J. Phys. Chem. A, 104(45):10321-10327, 2000.

[57] BR Johnson. The multichannel log-derivative method for scattering calculations. J. Comp. Phys, 14:445, 1973.

[58] F. Mrugała and D. Secrest. The generalized log-derivative method for inelastic and reactive collisions. J. Chem. Phys., 78:5954, 1983.

[59] DE Manolopoulos. An improved log derivative method for inelastic scattering. J. Chem. Phys., 85:6425, 1986.

[60] D.E. Manolopoulos and J.C. Light. A log derivative formulation of reaction rate theory. Chemical Physics Letters, 216(1-2):18-26, 1993.

[61] Evgeny Vetoshkin and Dmitri Babikov. Semiclassical wave packet study of ozone forming reaction. J. Chem. Phys., 125(2):024302, 2006. 
[62] Oleg L. Polyansky, Per Jensen, and Jonathan Tennyson. The potential energy surface of h[sub 2] [sup 16]o. J. Chem. Phys., 105(15):6490-6497, 1996.

[63] J.C. Tully. Molecular dynamics with electronic transitions. J. Chem. Phys., 93:1061, 1990.

[64] E. Neria and A. Nitzan. Semiclassical evaluation of nonadiabatic rates in condensed phases. J. Chem. Phys., 99(2):1109, 1993.

[65] O.V. Prezhdo and P.J. Rossky. Mean-field molecular dynamics with surface hopping. J. Chem. Phys., 107(3):825, 1997.

[66] M.D. Hack and D.G. Truhlar. A natural decay of mixing algorithm for nonBorn-Oppenheimer trajectories. J. Chem. Phys., 114:9305, 2001.

[67] Hans-Dieter Meyera and William H. Miller. A classical analog for electronic degrees of freedom in nonadiabatic collision processes. J. Chem. Phys., 70(7):3214-3223, 1979.

[68] G. Stock and M. Thoss. Semiclassical Description of Nonadiabatic Quantum Dynamics. Phys. Rev. Lett., 78(4):578-581, 1997.

[69] X. Sun. Semiclassical initial value representation for electronically nonadiabatic molecular dynamics. J. Chem. Phys., 106(15):6346, 1997.

[70] A.W. Jasper, M.D. Hack, A. Chakraborty, D.G. Truhlar, and P. Piecuch. Pho- 
todissociation of $\mathrm{LiFH}$ and $\mathrm{NaFH}$ van der Waals complexes: A semiclassical trajectory study. J. Chem. Phys., 115:7945, 2001.

[71] M. Topaler and N. Makri. Quantum rates for a double well coupled to a dissipative bath: Accurate path integral results and comparison with approximate theories. J. Chem. Phys., 101:7500, 1994.

[72] M. Thoss, H. Wang, and W.H. Miller. Generalized forward-backward initial value representation for the calculation of correlation functions in complex systems. J. Chem. Phys., 114:9220, 2001.

[73] N. Makri and W.H. Miller. Monte Carlo path integration for the real time propagator. J. Chem. Phys., 89:2170, 1988.

[74] JS Shao and N. Makri. Forward-backward semiclassical dynamics without prefactors. J. Phys. Chem. A, 103(39):7753-7756, 1999.

[75] Haobin Wang, Xueyu Song, David Chandler, and William H. Miller. Semiclassical study of electronically nonadiabatic dynamics in the condensedphase: Spin-boson problem with debye spectral density. J. Chem. Phys., 110(10):4828-4840, 1999.

[76] S. Garashchuk and J.C. Light. Simplified calculation of the stability matrix for semiclassical propagation. J. Chem. Phys., 113:9390, 2000. 